Aus der Klinik für Anästhesiologie

(Prof. Dr. med. Konrad Meissner)

der Medizinischen Fakultät der Universität Göttingen

\title{
Der Einfluss präoperativer Faktoren auf den postoperativen Schmerzverlauf nach Hüftgelenksersatz
}

\author{
INAUGURAL - DISSERTATION \\ zur Erlangung des Doktorgrades \\ der Medizinischen Fakultät der \\ Georg-August-Universität zu Göttingen
}

vorgelegt von

Merle Rose Luzie Marie Gathmann

aus

Oberhausen 
Dekan:

Referent:

Ko-Referent:

Drittreferent:
Prof. Dr. med. W. Brück

Prof. Dr. Frank Petzke

PD Dr. Thelonius Hawellek

Prof. Dr. Martin Oppermann

Datum der mündlichen Prüfung: 16.02.2021 
Hiermit erkläre ich, die Dissertation mit dem Titel "Der Einfluss präoperativer Faktoren auf den postoperativen Schmerzverlauf nach Hüftgelenksersatz" eigenständig angefertigt und keine anderen als die von mir angegebenen Quellen und Hilfsmittel verwendet zu haben.

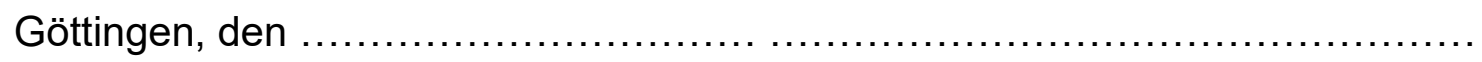




\section{Inhaltsverzeichnis}

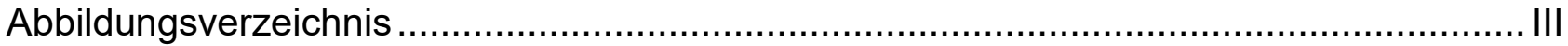

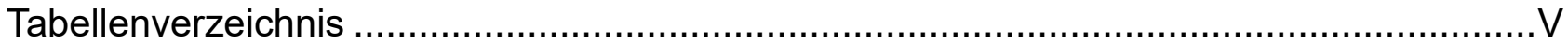

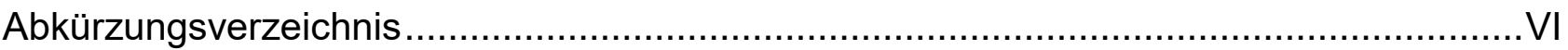

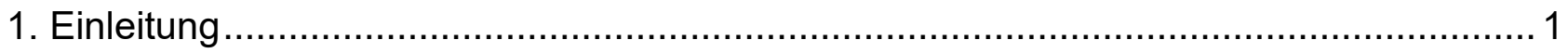

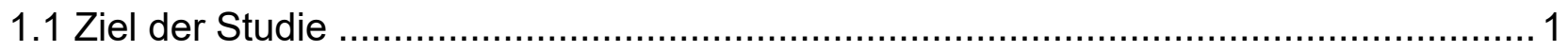

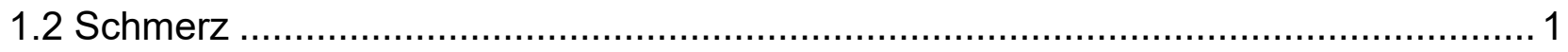

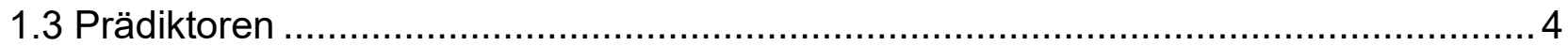

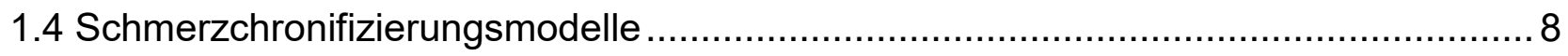

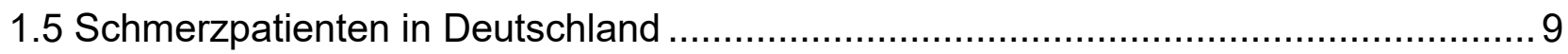

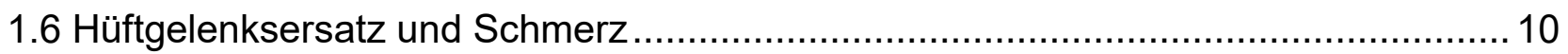

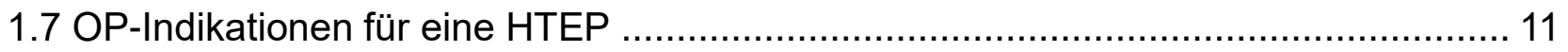

1.8 Anforderungen an einen chirurgischen Eingriff ................................................. 11

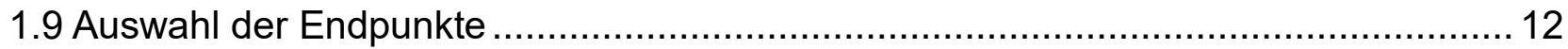

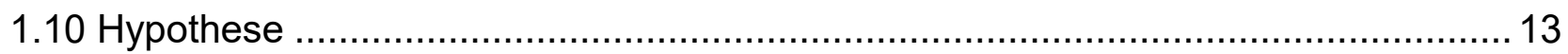

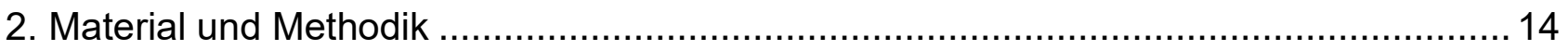

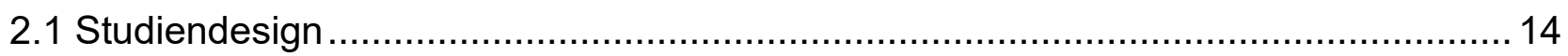

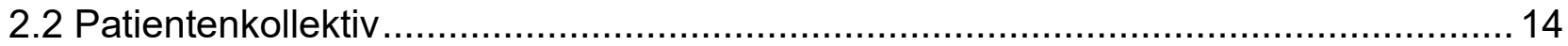

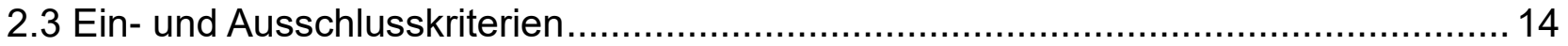

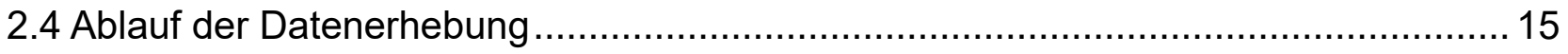

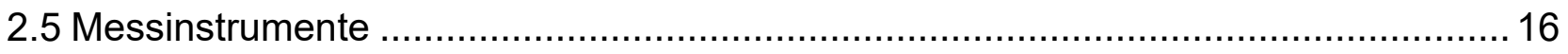

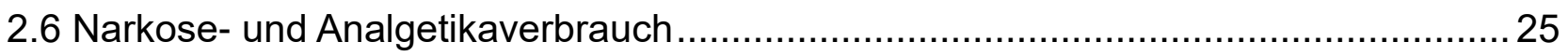

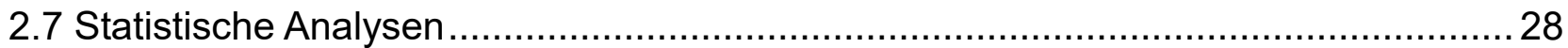

3. Ergebnisse

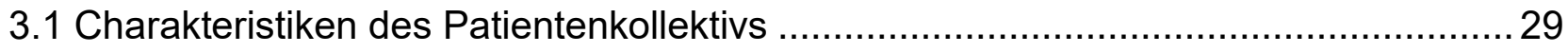

3.2 Beschreibung der präoperativ erhobenen Einflussfaktoren .................................... 32 


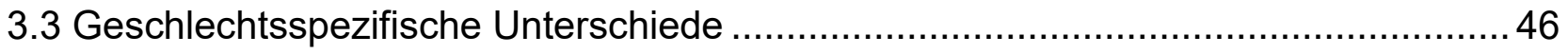

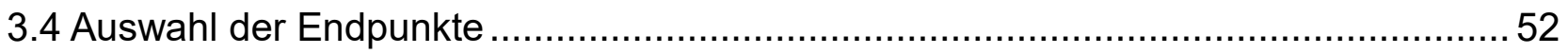

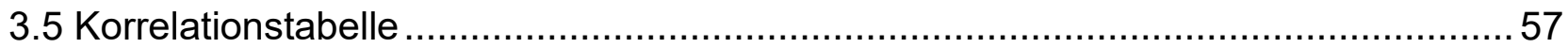

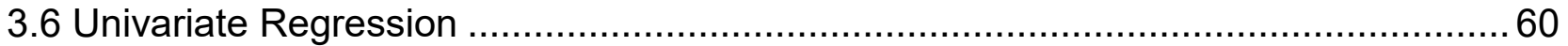

3.7 Ausschlüsse von Variablen für die multiple Regression .......................................... 65

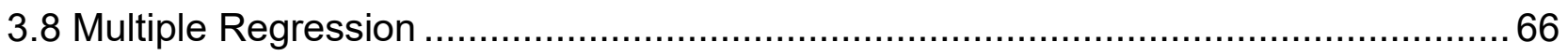

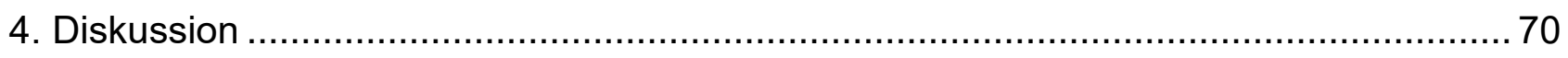

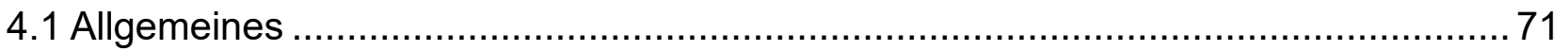

4.2 Präoperativer Morphin-, Analgetika- und Koanalgetikakonsum .............................. 71

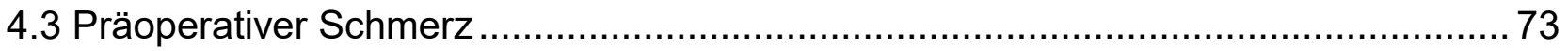

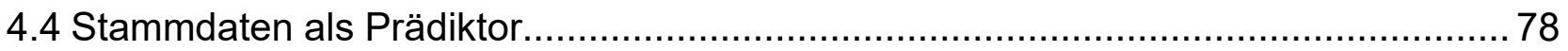

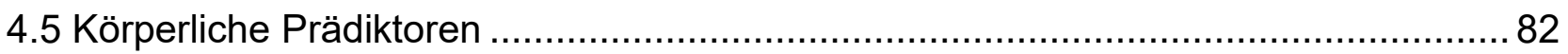

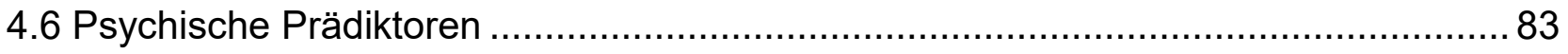

4.7 Angewandte Methodik und Analgesie im Annastift Hannover .................................. 89

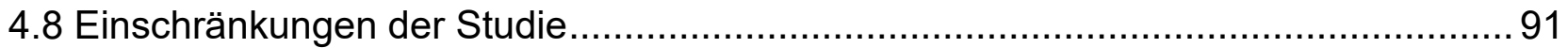

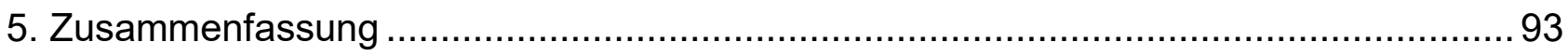

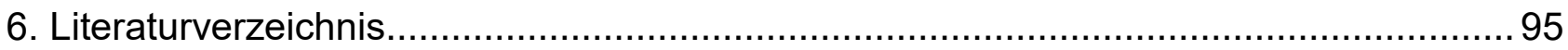




\section{Abbildungsverzeichnis}

Abbildung 1: Schmerzmedikationsschema Hannover ............................. 27

Abbildung 2: Flowchart Patientenkollektiv ........................................ 30

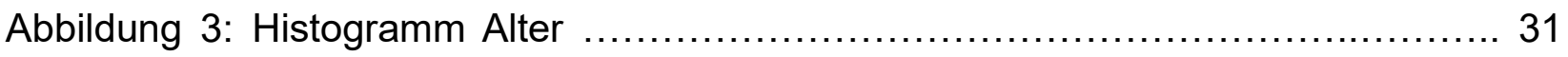

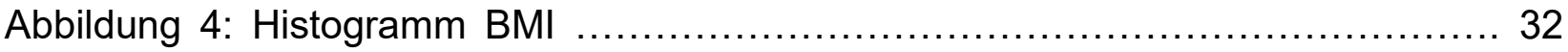

Abbildung 5: Histogramm DASS-Depression ....................................... 33

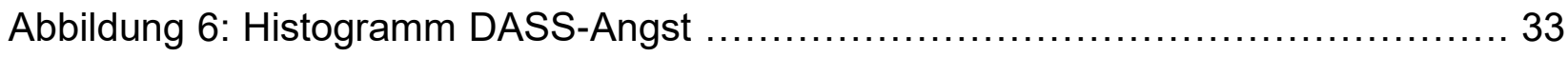

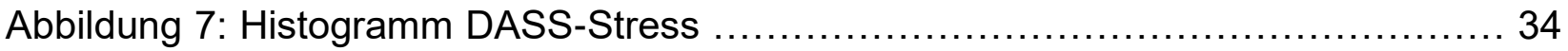

Abbildung 8: Histogramm KSI Hilfe und Hoffnungslosigkeit ........................... 35

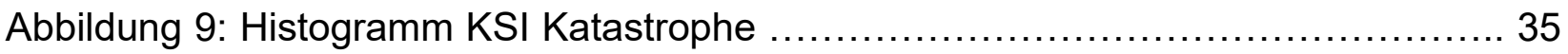

Abbildung 10: Histogramm KSI Durchhalteappell .................................... 36

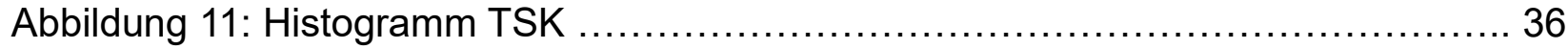

Abbildung 12: Histogramm SF12 Körperliche Summenskala ........................ 37

Abbildung 13: Histogramm SF12 Psychische Summenskala ....................... 38

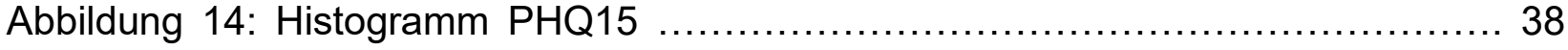

Abbildung 15: Histogramm WOMAC-Schmerz ..................................... 39

Abbildung 16: Histogramm WOMAC-Steifigkeit ................................... 39

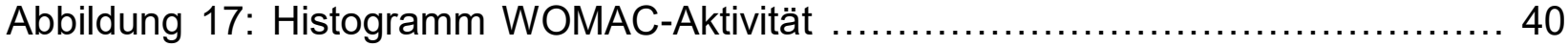

Abbildung 18: Histogramm Schmerzschwelle ..................................... 40

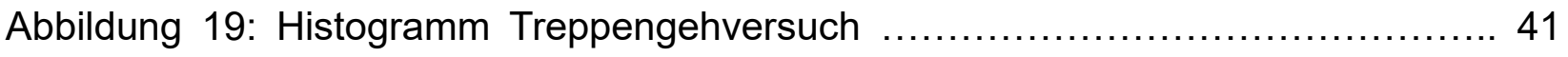

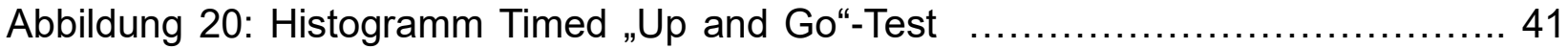

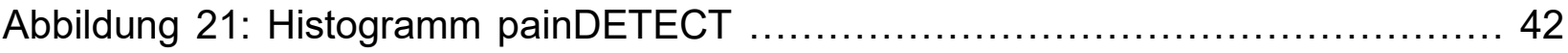

Abbildung 22: Histogramm MQS „Vorbehandlung mit Opioiden“ .................... 43

Abbildung 23: Histogramm MQS „Vorbehandlung mit Nichtopioiden“ .................... 43

Abbildung 24: Scatterplot stärkster Schmerz vs. stärkste Hüftschmerz in den letzten

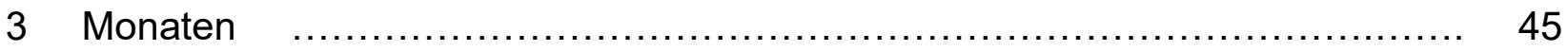

Abbildung 25: Scatterplot durchschnittlicher Schmerz vs. Durchschnittlicher Hüftschmerz

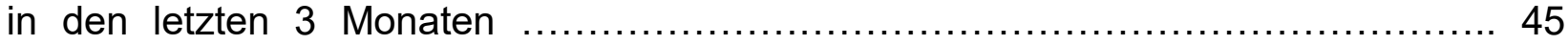

Abbildung 26: Histogramm durchschnittlicher Schmerz in den letzten 3 Monaten .... 46

Abbildung 27: Boxplot Geschlechtervergleich Schmerzschwelle in kPA ............. 48

Abbildung 28: Boxplot Geschlechtervergleich WOMAC Schmerz ...................... 49 
Abbildung 29: Boxplot Geschlechtervergleich beim DASS-Depression .............. 50

Abbildung 30: Boxplot Geschlechtervergleich beim DASS-Angst .................... 51

Abbildung 31: Boxplot Geschlechtervergleich beim DASS-Stress .................... 51

Abbildung 32: Scatterplot stärkster Schmerz \& Belastungsschmerz am 1. pOP Tag

Abbildung 33: Scatterplot stärkster Schmerz \& Belastungsschmerz am 5. pOP Tag

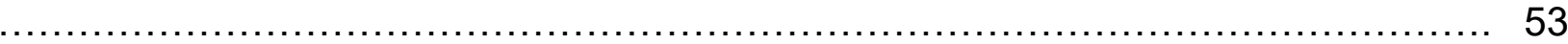

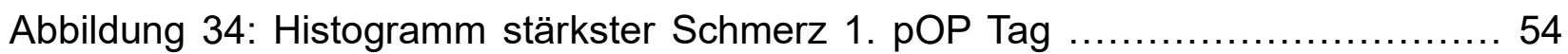

Abbildung 35: Histogramm stärkster Schmerz 5. pOP Tag …........................54

Abbildung 36: Histogramm Morphinäquivalent 48 h pOP ............................ 55

Abbildung 37: Histogramm Morphinäquivalent 5. pOP Tag …...................... 55

Abbildung 38: Korrelation stärkster Schmerz \& Morphinäquivalent 1. pOP Tag ...... 56

Abbildung 39: Korrelation stärkster Schmerz \& Morphinäquivalent 5. pOP Tag ..... 56

Abbildung 40: Scatterplot stärkster Schmerz 1. pOP Tag vs. Alter .................... 62

Abbildung 41: Scatterplot stärkster Schmerz 5. pOP Tag vs. painDETECT ........... 63

Abbildung 42: Scatterplot stärkster Schmerz 5. pOP Tag vs. Alter ................... 64

Abbildung 43: Scatterplot Morphinäquivalent $48 \mathrm{~h}$ vs. Alter ........................ 65

Abbildung 44: Scatterplot stärkster Schmerz 1. pOP Tag vs. DASS-Stress ..........68 68

Abbildung 45: Boxplot stärkster Schmerz 5.pOP Tag vs. Timed „Up and Go“-Test ... 68

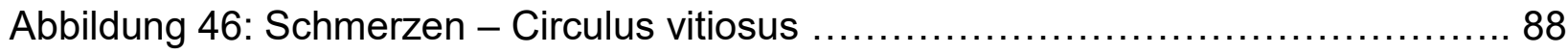




\section{Tabellenverzeichnis}

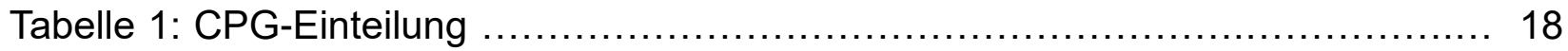

Tabelle 2: Unterschiede der einzelnen Schmerzskalen in den letzten 3 Monaten ....... 44

Tabelle 3: Geschlechtsspezifische Unterschiede ................................... 47

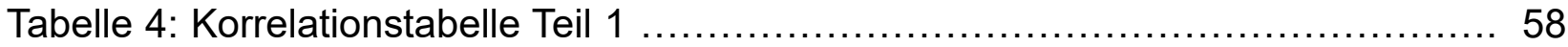

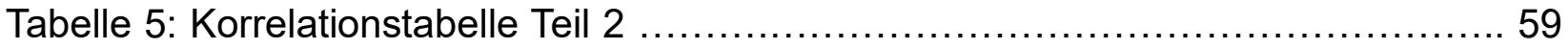

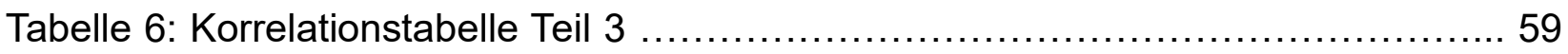

Tabelle 7: Univariate Regression: Eingangsparameter mit den gewählten Endpunkten

Tabelle 8: Aufstellung aller signifikanten univariaten Parameter für die multiple

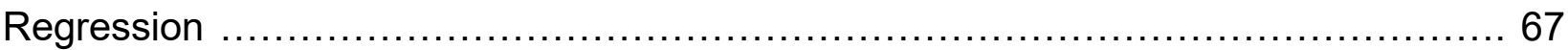

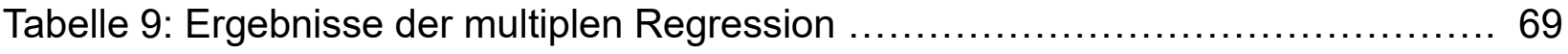

\section{Abkürzungsverzeichnis}

\begin{tabular}{|c|c|}
\hline BMI & Body-Mass-Index \\
\hline CPG & chronic pain grade \\
\hline DASS & depression anxiety and stress scale \\
\hline DNIC & diffuse noxious inhibitory control \\
\hline durchs. & durchschnittlicher/durchschnittliche/durchschnittliches \\
\hline HTEP & totale Hüftgelenksendoprothese \\
\hline $\mathrm{kPa}$ & Kilopascal \\
\hline KSI & Kieler Schmerz-Inventar \\
\hline K.S.-Test & Kolmogorov-Smirnov- Anpassungstest \\
\hline LJ & Lebensjahr \\
\hline MPSS & Mainzer Stadienmodell der Schmerzchronifizierung \\
\hline MQS & medication quantification scale \\
\hline $\mathrm{PHQ}$ & patient health questionnaire - Gesundheitsfragebogen für Patienten \\
\hline pOP & postoperativ/postoperativer/postoperativem/postoperativen \\
\hline QUIPS & Qualitätsverbesserung in der postoperativen Schmerztherapie \\
\hline SF & short form \\
\hline TSK & tampa scale for kinesiophobia \\
\hline WOMAC & Western Ontario and McMaster universities osteoarthritis Index \\
\hline
\end{tabular}




\section{Einleitung}

\subsection{Ziel der Studie}

Trotz intensiver Bemühungen und des technischen medizinischen Fortschrittes fallen immer wieder Patienten mit starken postoperativen Schmerzen auf. Neben der Optimierung des perioperativen Schmerzmanagements stellt sich die Frage, welche besonderen individuellen Merkmale diese etwa 20-40 \% der Patienten haben, wie diese den postoperativen Schmerz verstärken oder mindern können und wie man diese ggf. im perioperativen Ablauf beeinflussen kann (Gerbershagen et al. 2013). Studien zu individuellen Risikofaktoren beschränken sich meistens auf einzelne Merkmale, wie Depression, oder Merkmalsgruppen, wie psychologische Faktoren, und befassen sich selten mit dem Gesamtbild (Macrae 2001; Perkins und Kehlet 2000).

Ziel dieser Studie war es, sowohl klinische, psychische als auch physiologische Risikofaktoren präoperativ zu identifizieren und $\mathrm{zu}$ verschiedenen Zeitpunkten postoperativ den Einfluss auf die Schmerzintensität und den Schmerzmittelkonsum zu bestimmen. In dieser Doktorarbeit wird der akute postoperative Schmerz nach elektiver Hüftendoprothese genauer betrachtet.

\subsection{Schmerz}

Schmerz kann nach verschiedenen Gesichtspunkten eingeteilt werden. In einer zeitlichen Dimension kann man den akuten Schmerz vom chronischen Schmerz unterscheiden.

\subsubsection{Akutschmerz}

Der Akutschmerz dient als Warnfunktion, um den Organismus über drohende oder bereits eingetretene Verletzungen $\mathrm{zu}$ informieren und ist zeitlich terminiert. Zum Akutschmerz gehören der nozizeptive Schmerz, der inflammatorische Schmerz und eingeschränkt auch der neuropathische Schmerz. Er wird über Nozizeptoren auf freien Nervenendigungen registriert und über markarme C- und gering myelinisierte a-deltaFasern ans 2. Neuron weitergeleitet (Schulz und Locher 2013). 
Der nozizeptive Schmerz, welcher beispielsweise durch einen starken mechanischen Reiz oder eine chemische Noxe entsteht, hat eine Warnfunktion, die bei intakter Schmerzwahrnehmung den Organismus über Gefahren informiert. Durch die Gewebsverletzung werden Nozizeptoren aktiviert, die solange aktiv sind, bis die Noxe ausgeschaltet oder beseitigt ist (Kehlet et al. 2006).

Der inflammatorische Schmerz wird u. a. durch freigesetzte Entzündungsmediatoren und Neurotransmitter vermittelt. Die Wahrnehmungsschwelle der Nozizeptoren wird dadurch gesenkt, und es kommt zu einer peripheren Sensibilisierung. Die Nozizeptoren reagieren also schneller auf Reize, zum Beispiel im Bereich einer Wunde. Zusätzlich kommt es durch die erhöhte periphere Erregbarkeit auch zu einer zentralen Sensibilisierung, was eine Hypersensibilität nicht nur im Bereich der Wunde zur Folge hat, sondern auch in unmittelbar benachbarten Arealen des Wundgebietes. Der verursachte Schmerz kann Stunden bis Tage anhalten, er bleibt in der Regel solange bestehen, bis die Entzündung ausgeheilt ist und keine Entzündungsmediatoren oder Transmitter die Erregbarkeit weiter steigern. Die Sensibilisierungsvorgänge zentral und peripher sind dabei meist komplett reversibel (Kehlet et al. 2006; Schnabel und Pogatzki-Zahn 2010).

Der neuropathische Schmerz wird hingegen durch eine Schädigung sensibler Strukturen des peripheren Nervens oder des ZNSs verursacht. Dies führt zu einem peripheren Verlust der Sensibilität, wie z. B. von Temperatur oder Schmerz, und kann zusätzlich mit einer paradoxen Hypersensibilität oder Allodynie einhergehen, wo beispielsweise leichte Berührungen bereits Schmerzen verursachen. Andererseits besteht oft eine Überempfindlichkeit auf Schmerzreize (Hyperalgesie) (Kehlet et al. 2006; Schulz und Locher 2013; Schnabel und Pogatzki-Zahn 2010).

\subsubsection{Chronischer bzw. persistierender Schmerz}

Aus jeder Art von Akutschmerz kann sich jedoch ein chronischer Schmerz entwickeln. In diesem Fall hat der Schmerz die Funktion als Schadensmelder verloren und hat bei ungünstiger Entwicklung zunehmend negative Folgen auf die Lebensqualität Betroffener und wird krankheitsbestimmend. Diese Kombination aus körperlichen und 
psychologischen Veränderungen bezeichnet man dann auch als Schmerzkrankheit. Diese zu behandeln stellt eine große Herausforderung an die aktuelle Schmerzbehandlung dar, da die vielfältigen Ursachen oft schwer zu detektieren sind und die Patienten häufig stark unter ihren Schmerzen leiden.

Es ist immer noch schwierig, den Begriff chronischer Schmerz genau zu definieren. Synonym verwendet man gerade im Bereich chronischer postoperativer Schmerzen auch das Wort persistierend, um den negativen Aspekt, welchen das Wort chronisch beinhaltet, zu vermeiden.

Zum einen gibt es die Möglichkeit, die Schmerzentwicklung rein auf der Zeitachse zu betrachten. In diesem Fall gilt ein Patient als Schmerzchronifiziert, wenn ein Patient nach drei Monaten (Lavand'homme und Progatzki-Zahn 2017) immer noch Schmerz empfindet. Manche Autoren verwenden auch größere Zeitspannen.

Zum anderen gibt es einen klaren Bezug zu ursächlichen Erklärungen, wie beispielsweise eine Operation. In diesem Fall spricht man von chronischem postoperativen Schmerz, wenn ein Patient zwei (Macrae 2001) bzw. drei (Gerbershagen et al. 2013) Monate nach der Operation weiterhin Schmerzen in Folge der Operation im operierten Bereich empfindet. Nach Operationen schwankt die Zahl der Patienten mit persistierenden Schmerzen je nach Eingriff zwischen 10 bis 50\%. Nur etwa ca. 2-10\% aller Patienten entwickeln starke bis sehr starke Schmerzen und zeigen eine ernsthafte Schmerzchronifizierung. Es gibt für chronische postoperative Schmerzen sowohl intraoperative Faktoren, als auch prä- und postoperative Faktoren (Perkins und Kehlet 2000). Ursächlich diskutiert werden u. a. intraoperative Faktoren, wie Gewebsschäden und nervale Verletzungen (Kehlet et al. 2006). Aber auch mit der Dauer der durchgeführten OP steigt das Chronifizierungsrisiko (Peters et al. 2007). Andererseits geben etwa $20 \%$ der Patienten in Schmerzkliniken eine chirurgische Intervention als Ursache an (Gerbershagen 2013).

Der operativ (iatrogen) ausgelöste neuropathische Schmerz ist eine der häufigsten Ursachen für persistierende postoperative Schmerzen. Dieser Risikofaktor kann zwar 
mit einer guten und minimalinvasiven chirurgischen Behandlung stark reduziert, aber nicht komplett vermieden werden (Kehlet et al. 2006).

\subsection{Prädiktoren}

Die eine erklärende Ursache für eine Schmerzchronifizierung gibt es demnach nicht. Schmerz wird multifaktoriell sowohl von psychischen, physischen als auch sozialen Faktoren beeinflusst (Turk und Okifuji 1996).

Auch bei chronisch postoperativen Schmerzen gibt es neben den direkten chirurgischen Zusammenhängen auch bereits identifizierte prä- und postoperative Risikofaktoren, wie eine genetische Komponente, das weibliche Geschlecht, das Alter, psychosoziale Faktoren und Präsenz präoperativer Schmerzen, die eine Chronifizierung wahrscheinlicher machen (Nikolajsen et al. 2006).

Nach Liu et al. 2012 ist der präoperative Analgetika- und Koanalgetikgebrauch insbesondere ein Prädiktor für akute postoperative Schmerzen. Vielfältige weitere Faktoren für starke akute postoperative Schmerzen wurden bereits identifiziert, allerdings meist in Studien mit gezielt gewählten einzelnen Parametern (Ip et al. 2009).

\subsubsection{Physische Prädiktoren}

Die Stärke bzw. Intensität des Schmerzes ist noch kein Zeichen für das Ausmaß der Schädigung (von Korff et al. 1992). Genauso wenig kann man anhand der Dauer der Akutschmerzen die chronischen Schmerzen vorhersagen. Aber ein starker postoperativer Akutschmerz korreliert mit einem erhöhten Entwicklungsrisiko von persistierenden Schmerzen, also einer Schmerzchronifizierung (Kehlet et al. 2006; Schnabel und Pogatzki-Zahn 2010) und verzögert auch die Heilung (Gouin und KiecoltGlaser 2011).

Bei unbehandeltem postoperativem Schmerz sinkt zudem die Patientenzufriedenheit, ein Anstieg sowohl der Mortalität als auch der Morbidität wird diskutiert (Khan et al. 2011). 
So gelten Schmerzen direkt nach einem operativen Eingriff im Aufwachraum als ein Prädiktor für Schmerzen während des stationären Aufenthaltes. Schmerzen während des stationären Aufenthaltes wiederum gelten als Prädiktor für Schmerzen nach der Entlassung, beispielsweise im häuslichen Umfeld (Kain et al. 2000).

Die Schmerzschwelle präoperativ schien in einigen Studien auch ein Prädiktor für die Stärke von postoperativen Schmerzen zu sein. Je höher die Schmerzschwelle, desto niedriger die Schmerzen. Dabei wurde die Schmerzschwelle in den verschiedenen Studien mit diversen Methoden erfasst. So gibt es thermische und elektrische Reize sowie auch die mechanische Druckschmerzschwelle, ausgeführt mit einem handgeführten Algometer (Abrishami et al. 2011). In aktuellen Studien wurde wiederholt ein Zusammenhang zwischen einer reduzierten körpereigenen Schmerzhemmung und dem Auftreten chronischer postoperativer Schmerzen nachgewiesen (van Laarhoven et al. 2007; Petersen et al. 2015).

Es gibt Übersichtsarbeiten, die schlussfolgern, dass ein verminderter postoperativer Schmerz auch mit einer erniedrigten Komplikationsrate einhergeht. So haben die Patienten kurzfristig gesehen ein geringeres Risiko eines thrombembolischen Ereignisses, einer Lungenbeteiligung und insbesondere auch ein geringeres Mortalitätsrisiko nach einer HTEP-Operation zu erlangen (Huang et al. 2001).

Die präoperative körperliche Aktivität ist bei HTEP-Patienten deutlich vermindert und verbessert sich meist mit einer Operation (Nilsdotter et al. 2003). Patienten, die präoperativ stärker eingeschränkt waren, hatten auch postoperativ schlechtere Ergebnisse, sowohl was ihre Funktionalität als auch ihre Schmerzen betraf (Fortin et al. 1999).

Was die Krankenhausaufenthaltsdauer betrifft, so wurden insbesondere die Patienten früher entlassen, welche präoperativ schnell vom Sitzen aufstehen und im Anschluss 3 Meter laufen konnten (Schneider et al. 2009). 


\subsubsection{Psychische Prädiktoren}

Wie bereits angeführt, haben auch viele psychische Faktoren Einfluss auf die Schmerzwahrnehmung. Eine psychische Beeinflussung auf akute Schmerzen ist eher wahrscheinlich, allerdings kann vom Fehlen einer orthopädischen Ursache nicht unmittelbar auf eine psychische Ursache geschlossen werden (Pacault-Legendre et al. 2009).

So findet man häufig eine Komorbidität von Depression und chronischen Schmerzen. Ob dabei die Depression oder der Schmerz die initiale Ursache darstellen ist dabei häufig schwer zu identifizieren (Wylde et al. 2011 b).

Nimmt man als Erklärungsmodell zuerst die Depression und dann den Schmerz an, so wird diese Theorie zum Beispiel durch eine Studie unterstützt, in der depressive Verstimmungen bereits präoperativ bei nicht chronifizierten Schmerzpatienten festgestellt wurden und diese Patienten postoperativ dann häufiger unter chronischen Schmerz litten (Brander et al. 2003).

Patienten, die präoperativ Antidepressiva genommen haben, bekamen mit einer höheren Wahrscheinlichkeit akute und chronische postoperativen Schmerzen (Liu et al. 2012; Luman et al. 1996).

Häufig findet man Korrelationen von depressiver Verstimmung mit Angst und einer Somatisierungsneigung. Diese drei psychischen Auffälligkeiten sind besonders häufig bei Schmerzpatienten (Kroenke et al. 2002).

Des Weiteren gibt es allerdings auch eine Angst-Stress-Verbindung. Kain et al. richteten dabei das Hauptaugenmerk auf die Angstkomponente (Kain et al. 2000). Die Stresskomponente scheint aber auch selber Einfluss auf postoperative Schmerzen zu haben (Geiss et al. 2005). Jedoch wird Stress dabei meistens verschieden operationalisiert und isoliert betrachtet, sowie beispielsweise der berufliche Stress, die Bestimmung von Cortisol als Biomarker, oder direkt der gesamte Bedrängnisgrad einer Person (Geiss et al. 2005; Kain et al. 2000; Munafo und Stevenson 2001). 


\subsubsection{Stammdaten als Prädiktoren}

Stammdaten wie der BMI, das Geschlecht und das Alter wurden schon als verschiedene Prädiktoren bezüglich der postoperativen Schmerzen und des postoperativen Analgetikakonsums identifiziert (Singh und Lewallen 2010).

Im Alter benötigen Patienten weniger Schmerzmedikation, da ihr Stoffwechsel verlangsamt ist und ihre Multimorbidität häufig zu einem erhöhten Medikamentenkonsum generell führt, was wiederum den Abbau der Analgetika generell verlangsamen kann (AGS Panel on the Pharmacological Management of Persistent Pain in Older Persons 2009; Hanlon et al. 2009). Auch werden bei älteren Patienten derzeit eher Opioide empfohlen, um das Risiko eines durch NSAR-induziertes Ulcus ventriculi zu vermindern (Pivec et al. 2014). Allerdings haben auch Opioide relevante negative Effekte, gerade bei sehr alten Patienten (Solomon et al. 2010). Zusätzlich gibt es Studien, wo ein niedriges Alter selbst ein Risikofaktor für eine Schmerzchronifizierung ist. Auch geben jüngere Patienten zu einem höheren Prozentsatz stärkere Schmerzen an als ältere Patienten (Aasvang und Kehlet 2005; Liu et al. 2012).

Personen mit einem höheren BMI benötigen häufiger eine HTEP (Busato et al. 2008). $\mathrm{Ob}$ es an dem höheren Gewicht liegt, welches die Knochen belastet oder an einem begleitenden Bewegungsmangel und damit auch progredienten Knochendegeneration, ist schwer einzuordnen. Jedenfalls haben Patienten mit einem hohen BMI auch häufiger eine Osteoarthritis der Hüfte (Bijlsma et al. 2011). Ein hoher BMI verlängert zwar die Operationsdauer, aber laut mehrerer Studien nicht den Krankenhausaufenthalt, die Wundheilung oder die postoperativen Schmerzen (Batsis et al. 2009; Busato et al. 2008). Es gibt jedoch auch Studien die zeigen, dass der BMI als Prädiktor für postoperative Schmerzen und Analgetikabedarf postoperativ galt. Allerdings beziehen sich diese Studien nicht auf den direkten stationären Verlauf, sondern auf einen längerfristigen Einfluss (Singh und Lewallen 2010). Durch die erhöhte Gefahr einer Prothesenlockerung im Verlauf und durch eine verzögerte körperliche Funktionswiederherstellung ist die Komplikationsrate bei Übergewichtigen höher, sodass eine Gewichtsreduktion vor einer HTEP stark empfohlen wird sowie bei diesen Patienten 
auch eine absolute Ausschöpfung konservativer Maßnahmen anzustreben ist (Ibrahim et al. 2013; Innocenti et al. 2013).

Beim Geschlecht gibt es laut diverser Literatur verschiedene Ergebnisse. Häufig geben Frauen einen höheren NRS-Wert an, was aber bisher nicht als sicherer Indikator einer schlechteren Behandlung von Frauen gesehen wird (Etherton et al. 2014). Auch geben Frauen häufiger muskuloskelettale Schmerzen an (Ruau et al. 2012). Eine weitere Studie hat herausgefunden, dass Frauen 2 Jahre postoperativ mehr Analgetika konsumieren als Männer (Singh und Lewallen 2010).

\subsection{Schmerzchronifizierungsmodelle}

Es gibt verschiedene Modelle zur Erklärung der Schmerzchronifizierung, die nicht als exklusiv verstanden werden sollten, sondern als mögliche Ursachen mit großer interindividueller Relevanz und Ausprägung. Es gibt verschiedene Angst-VermeidungsModelle, welche sich etwas voneinander unterscheiden (Etherton et al. 2014). Bei einer Version dieses Modell entscheidet der Patient (oft auch unbewusst), ob er sich seiner Angst stellt, sich bewegt und demzufolge in näherer Zukunft sehr wahrscheinlich eine Schmerzreduktion erfährt, oder ob der Patient z.B. manche Bewegungen nicht mehr ausführt oder vermeidet, aus Angst, die Schmerzen könnten sich verschlimmern. Der zweite Weg kann allerdings im Verlauf zu einer Fehlhaltung oder Fehlbewegung führen. Eine Fehlbewegung kann auf Dauer persistieren, wenn sich der Patient an seinen anderen Bewegungsablauf gewöhnt hat (Vlaeyen et al. 1995). Die Fehlhaltung selbst kann allerdings auch Schmerzen verursachen, oder es kommt zu einer Bewegungseinschränkung, was sekundäre muskuläre und motorische Störungen zur Folge haben kann. In diesem Fall bleibt der Schmerzgrad erhalten oder verschlimmert sich noch. Diese Modelle berücksichtigen sowohl eine kognitive als auch eine funktionelle Verhaltensform (Lethem et al. 1982; Vlaeyen und Linton 2000).

Schaut man sich andere Erklärungsmodelle an, so findet man viele Untersuchungen über die Rolle von Katastrophisieren in der Verarbeitung von Schmerzen. Mögliche Folgen einer bestimmten Bewegung oder körperliche Belastungen, wie eine Schmerzverstärkung, werden aus dem Kontext heraus als bedrohlich und extrem 
negativ erfahren (,immer muss das mir passieren“, ,jetzt kann man gar nichts mehr tun“). Das Gefühl von Hilflosigkeit und Verlust der Kontrolle verschlimmert eher die gegenwärtige Situation des Patienten. Dies führt letztlich dazu, dass die Patienten auch höhere Schmerzen angeben (Sullivan et al. 1998).

Stark ausgeprägtes Durchhaltevermögen kann ebenfalls zu einer Schmerzchronifizierung beitragen. Man vermutet, dass durch muskuläre Anspannungen und starke Verspannungen dem Körper zusätzlich starke Belastungen zugeführt werden, welche zu dauerhaften Schmerzen führen können. Fehlende Pausen und übermäßige Belastungen verstärken diesen Zusammenhang (Hasenbring et al. 2001).

Das Grundprinzip, dass es sich bei Schmerzchronifizierung um ein biopsychosoziales Geschehen handelt, wird von Modellen, wie dem Angst-Vermeidungs-Modell oder dem Katastrophisierungs-Modell, durch das Aufzeigen konkreter Mechanismen bestätigt.

\subsection{Schmerzpatienten in Deutschland}

In Deutschland geben laut Häuser et al. 26,3 \% der Bevölkerung an, unter chronischen oder regelmäßigen Schmerzen in den letzten 3 Monaten ohne einer malignen Erkrankung zu leiden (Häuser et al. 2014). Laut einer Publikation von Breivik et al. 2006 geben nur $10 \%$ der Deutschen mit chronischen Schmerzen an, dass sie zu einem Schmerzspezialisten gegangen sind. Auch scheinen $53 \%$ der chronischen Schmerzpatienten in Europa keine verschreibungspflichtige Medikamente ein zu nehmen, wobei $61 \%$ des deutschen Patientenkollektivs aussagen, dass die Medikation inadäquat sei. $21 \%$ der Europäer behaupten niemals verschreibungspflichtige Medikamente genommen zu haben und $26 \%$, die einmal verschreibungspflichtige Medikamente genommen haben, nahmen diese zum Zeitpunkt der Erhebung nicht mehr ein. In Deutschland geben die Patienten an, dass $54 \%$ der verschreibungspflichtigen Substanzen NSAR sind, 2 \% Paracetamol, $8 \%$ COX-2-Inhibitoren, $20 \%$ schwache Opioide und $4 \%$ starke Opioide. Im europäischen Vergleich sind das deutlich mehr NSARs, aber entsprechend weniger Paracetamol. Die übrigen Substanzen sind Koanalgetika, wie Barbiturate und Antidepressiva (Breivik et al. 2006). 


\subsection{Hüftgelenksersatz und Schmerz}

Ziel einer HTEP ist eine Verbesserung der Lebensqualität, eine Verminderung der Schmerzen und bessere körperliche Aktivität (Montin et al. 2008).

Ca. $20 \%$ der deutschen Schmerzpatienten in der Studie von Frettlöh et al. 2009 geben Muskel-, Gelenk- und Knochenschmerzen als Hauptschmerzlokalisationen an. Als Hauptursache nannten 44 \% körperliche Belastung.

Betrachtet man die Daten aus Europa, so geben ca. $8 \%$ der chronischen Schmerzpatienten als Hauptlokalisation den Hüftschmerz an (Breivik et al. 2006). Allerdings haben laut einer dänischen Studie ca. $12 \%$ der Patienten, welche sich einer HTEP- Operation unterzogen haben, eine Schmerzchronifizierung 12-18 Monaten nach der Intervention (Nikolajsen et al. 2006). Wie stark die Schmerzen waren, ist in dieser Veröffentlichung jedoch nicht zu erkennen. Eine andere Studie aus England zeigt, dass 27 \% der HTEP Patienten nach 3-4 Jahren noch unter Schmerzen leiden, jedoch nur 6 $\%$ starke persistierende Schmerzen angeben. Die anderen haben nur noch milde Schmerzen und erleben häufig eine Verbesserung zum vorherigen präoperativen Schmerz. Insgesamt scheinen bei diesem Patientenkollektiv auch nur $1 \%$ unter neuropathischen Schmerzen zu leiden (Wylde et al. 2011 b).

Frauen scheinen insgesamt häufiger eine HTEP zu benötigen als Männer. Dies liegt unter anderem an der steigenden Osteoporose nach der Menopause. Allerdings werden Frauen in England im Gegensatz zu Männern spärlicher zu einem Spezialisten geschickt und entsprechend auch seltener operiert. Zudem scheinen Frauen auch später einer Operation einzuwilligen. Ob es daran liegt, dass sie zuhause nicht fehlen wollen oder einfach länger die Beschwerden ertragen, oder ob es noch andere Gründe gibt, ist dabei nicht untersucht worden (Jüni et al. 2010).

Zusätzlich haben stark beeinträchtigte präoperative körperliche Funktionen einen Einfluss auf das postoperative funktionelle Outcome (Kessler und Käfer 2007). 


\subsection{OP-Indikationen für eine HTEP}

Bevor man einen endoprothetischen Hüftgelenksersatz durchführt, sollte man sowohl die zur Verfügung stehenden medikamentösen als auch physiotherapeutischen Maßnahmen vollkommen ausschöpfen. Zudem sollte auch das Alter berücksichtigt werden, da man erst ab dem 60LJ eine elektive HTEP empfiehlt. Vor dem $50 \mathrm{LJ}$ ist ein solcher Eingriff nur in Ausnahmesituationen empfohlen (Innocenti et al. 2013).

Der Nachweis eines organischen, strukturellen Schadens auf dem Röntgenbild ist nicht ausreichend als Indikation für eine totale Hüftendoprothese, da nur $9 \%$ der Männer und $11 \%$ der Frauen mit Osteoarthritis auch symptomatisch sind (Pivec et al. 2012). Ursächlich für Osteoarthritis in der Hüfte sind u.a. genetische Faktoren, körperliche Aktivität, vorherige Verletzungen, intensiver Sport, Alter und der BMI (Bijlsma et al. 2011).

Zudem haben $40 \%$ trotz eines röntgenologisch nachweisbaren Schadens keine Symptome und benötigen daher keinen Gelenksersatz (Innocenti et al. 2013). Primär stehen Schmerzen und eingeschränkte Funktion als Hauptindikationen im Vordergrund, da diese die Lebensqualität erheblich beeinflussen (Ibrahim et al. 2013). Je nach Land gibt es verschiedene Gewichtungen, wann eine Operation durchgeführt werden soll, allerdings berücksichtigen sie zumeist die Parameter Funktionsbeeinträchtigung, Schmerzen und röntgenologisch nachweisbarer Defekt (Birrell et al. 1999).

Mögliche absolute Kontraindikation ist eine lokale oder systemische Infektion, welche vorher unbedingt ausgeschlossen werden sollte. Weitere relative Kontraindikationen sind beispielsweise eine manifeste und schwere depressive Episode, Demenz oder Alkoholmissbrauch. In diesen Fällen kann nach dem Ermessen des Operateurs trotzdem eine HTEP eingesetzt werden (Innocenti et al. 2013).

\subsection{Anforderungen an einen chirurgischen Eingriff}

Die Anforderungen, eine gute perioperative und nicht nur orthopädische Behandlung bei den HTEPs zu gewährleisten, steigen zusehends. Die Operationen sollen schneller und gleichzeitig gewebs- und nervenschonender durchgeführt werden, Patienten 
frühestmöglich schmerzarm mobilisiert und auch schnellstmöglich entlassen werden (Ibrahim et al. 2013).

Behandlungsstrategien zum Hüftersatz unterscheiden sich zwischen operativen Zentren. Um möglichst standardisierte Operationsdurchführungen zu gewährleisten, erhoben wir nur Daten in dem orthopädischen Krankenhaus Annastift in Hannover. Somit verringerten wir die Einflussfaktoren von verschiedenen Operationstechniken. Allerdings wurden in diesem Krankenhaus nur elektive Operationen durchgeführt, was das Patientenklientel für die gewählte Fragestellung zwar bereits im Vorfeld selektierte, aber auch die Variabilität prä-und postoperativ reduzierte.

Vor der Operation soll eine ausgiebige Aufklärung der Patienten erfolgen. Dies führt laut verschiedener Studien zu einem besseren Outcome (Ibrahim et al. 2013). Auch die Patientenzufriedenheit hat einen großen Einfluss auf die Genesung, was eine gute Schmerztherapie postoperativ voraussetzt, da u.a. Schmerzen die Lebensqualität stark beeinflussen (Myles et al. 2000).

Unter Berücksichtigung dieser vielfältigen Einflüsse und im Bemühen einer guten Charakterisierung der individuellen Schmerzsymptomatik, um inn effektiver behandeln zu können, wird eine umfassende Schmerzanamnese zu Beginn der Behandlung präoperativ empfohlen (Schulz und Locher 2013).

Wichtig ist eine effektive Schmerzbehandlung des postchirurgischen Schmerzes zur Vermeidung starker Schmerzen. Dies führt neben der Patientenzufriedenheit zu einer Förderung der Heilung und beschleunigt die Rückkehr in den Alltag und ggf. auch in das Berufsleben (Althaus et al. 2012, Macrae 2001).

\subsection{Auswahl der Endpunkte}

Um eine Auswahl möglicher Endpunkte innerhalb der ersten postoperativen Woche zu treffen, wurde der Umstand der ursächlichen Operation berücksichtigt. Am ersten postoperativen Tag ist der Wundschmerz noch deutlich mitinbegriffen, und der Analgetika- und Opioidkonsum der ersten 48 Stunden schließt die Operation mit ein. Demgegenüber sind am 5. postoperativen Tag die Patienten bereits mobilisiert worden 
und die direkten Nachwirkungen des operativen Eingriffes vermindert. Des Weiteren konnte die Analgetika- und Opioidgabe bereits an den empfundenen Schmerz besser adaptiert werden.

So wurde einerseits der stärkste empfundene Schmerz am 1. und 5. postoperativen Tag, sowie der Analgetika- und Opioidverbrauch an den selbigen Tagen als Endpunkt bestimmt.

\subsection{Hypothese}

Ziel der Studie war es, mögliche präoperative physiologische, soziale, klinische und psychische Eigenschaften der Patienten zu charakterisieren und ihren Einfluss auf den direkten postoperativen stationären Schmerzverlauf und den Opioidbedarf (berechnet als Morphinäquivalent) nach einer HTEP-Operation zu untersuchen. Wir verwendeten dabei 2 Erhebungszeitpunkte. Zunächst den ersten postoperativen Tag, um den direkten Einfluss der Operation zu bestimmen und des Weiteren den 5. postoperativen Tag, an welchem die Patienten bereits mobilisiert wurden. Hierzu wurden zunächst einzelne Faktoren, wie Depression, Angst, Stress, Katastrophisieren, Hilfe- und Hoffnungslosigkeit, Durchhalteappell, Druckschmerzschwelle, Schmerzen der letzten 3 Monate, Analgetikakonsum, Aktivität mittels des Timed „Up and Go“-Tests und eines Treppengehversuches, Funktionalität mittels des WOMAC, Schmerzchronifizierung mittels MPSS und CPG sowie neuropathischer Schmerz und biometrische Daten univariat betrachtet, um zu prüfen, ob die Faktoren Einfluss auf den Opioidbedarf oder die Schmerzen postoperativ haben. Diese Faktoren sind in Vorstudien als entsprechend wichtige Einflussfaktoren identifiziert worden. In einem nächsten Schritt wurden die möglichen Einflussfaktoren multivariat überprüft.

Der Gedanke dabei ist, dass Schmerzen multifaktoriell beeinflusst werden und sinnvolle Parameter ggf. präoperativ erhoben werden sollten, um ein postoperatives optimiertes Therapieregime zu ergänzen. 


\section{Material und Methodik}

\subsection{Studiendesign}

Diese Studie war eine prospektive Kohortenstudie, wo bei Patienten mit einem elektiven einseitigen Hüftgelenksersatz die postoperativen Schmerzverläufe über eine Woche unter dem Gesichtspunkt möglicher präoperativen Einflussfaktoren betrachtet wurden.

Es wurde dabei nicht in die Behandlung selbst eingegriffen, sondern die klinischen Verläufe mithilfe von psychologischen und physiologischen Messparametern beobachtet, sowie Krankenverläufe aus den Akten entnommen.

Das genutzte Studienprotokoll wurde durch die Ethikkomission der Universitätsmedizin Göttingen (Nr. 5/4/12) und die Ethikkomission der Medizinischen Hochschule Hannover (Nr. 1483-2012) genehmigt.

Es wurden ausschließlich Patienten in die Studie eingeschlossen, welche die Ein- und Ausschlusskriterien, welche unter Punkt 2.3 genauer aufgeführt werden, erfüllten.

\subsection{Patientenkollektiv}

Für die Studie wurden alle Patienten, die sich in dem Zeitraum vom 23. Juli 2012 bis zum 28. Oktober 2012 im Diakoniekrankenhaus Annastift $\mathrm{GmbH}$ einem elektiven Hüftgelenksersatz unterzogen, gefragt, ob sie an der Studie teilnehmen wollten, sofern sie entsprechend den Ein- und Ausschlusskriterien grundsätzlich in Frage kamen.

\subsection{Ein- und Ausschlusskriterien}

In die Studie wurden volljährige Patienten, welche das 18. Lebensjahr erreicht hatten, unter freiwilliger Teilnahme aufgenommen, welche an der zu operierenden Hüfte einen Primäreingriff erhielten. Sie mussten mit der Nachbefragung nach 6 und 12 Monaten und der Bereitstellung von Kontaktdaten einverstanden sein.

Ausgeschlossen wurden Patienten, welche die deutsche Sprache in Schrift und Wort nicht verstanden. Zudem mussten die Patienten geistig und rechtlich in der Lage sein, nach Information und ärztlicher Aufklärung die Bedeutung/Konsequenzen Ihrer 
Studienteilnahme zu verstehen und in der Lage und willens sein, ihr schriftliches Einverständnis zur Teilnahme an der Studie zu geben. Patienten bei denen im Rahmen der Untersuchungen in der stationären Erfassungsphase eine konkrete suizidale Gefährdung festzustellen war, wurden für die Verlaufserfassung ausgeschlossen. Ebenso Patienten, bei denen es postoperativ $\mathrm{zu}$ Veränderungen im Bewusstseinszustand kam, oder bei denen schwere postoperative Komplikationen auftraten.

Des Weiteren wurden Patienten ausgeschlossen, welche sich für ein rückenmarksnahes Anästhesieverfahren entschieden oder anamnestisch einen Drogenabusus angaben.

Die Patienten durften jederzeit ihre Einwilligung zurückziehen.

\subsection{Ablauf der Datenerhebung}

Am Tag der Aufnahme wurden die teilnehmenden Patienten aufgeklärt und über die Studie informiert. Insofern sie eingewilligt hatten, wurde eine detaillierte Schmerzanamnese durchgeführt, zu der auch der Chronic Pain Grade (von Korff et al. 1992) und der painDETECT Fragebogen (Freynhagen et al. 2006) gehörten. Der MPSS (Gerbershagen 1986) wurde nach dem Anamnesegespräch vom Arzt bzw. von der Ärztin ausgefüllt. Nur für den painDETECT wurde am 7. postoperativen Tag eine Verlaufskontrolle durchgeführt.

Des Weiteren bekamen die Patienten am Tag der Aufnahme ein Fragebogenset, welches im Laufe des Tages von Innen beantwortet werden sollte. Eine Verlaufskontrolle mit teilweise auf den Befragunszeitraum angepassten Fragen wurde ebenfalls am 7. postoperativen Tag durchgeführt, um die Veränderungen nach der Operation zu ermitteln. Dieses Fragebogenset umfasst verschiedene schmerzpsychologische Testungen, die im Verlauf detailliert beschrieben werden.

Mittels einer klinischen Untersuchung wurden am Tag der Aufnahme die Bewegungsgrade der Patienten und im Laufe des Tages die Mobilität durch einen Treppengehversuch und dem Timed „Up and Go“-Test festgestellt. Inwieweit die Patienten ihre eigene Funktion selbst einschätzten, bedienten wir uns des WOMACs 
(Western Ontario and McMaster Universities Osteoarthritis Index) Fragebogen, der standardisiert im Anna-Stift durchgeführt wurde. Abgeändert und somit der Situation auf der Station nach der Operation angepasst, wurde dieser Fragebogen im Verlauf am 7. postoperativen Tag nochmals erfasst, um Veränderungen durch die Operation zu erkennen.

Zuletzt wurde am Tag der Aufnahme auch noch eine psychophysiologische Testung der Schmerzempfindlichkeit durchgeführt. Dabei wurde eine Schmerzschwellenmessung auf Druckreize und die DNIC (diffuse noxious inhibitory control) bestimmt. Diese Testung wurde am 3. postoperativen Tag wiederholt. Diese Daten werden hier nicht berichtet.

Nach der Operation und während des stationären Aufenthaltes bestimmten wir am 1., 3., 5., und zuletzt am 7. postoperativen Tag mithilfe des QUIPS (Qualitätsverbesserung in der postoperativen Schmerztherapie, Erklärung s.u.) den Schmerzintensitätsverlauf und das subjektive Empfinden der Patienten.

\subsection{Messinstrumente}

\subsubsection{Fragebogen Schmerzanamnese und Medikation}

Um detaillierte Informationen über den Schmerzcharakter identifizieren zu können, verwendeten wir den von der Deutschen Schmerzgesellschaft empfohlenen deutschen Schmerzfragebogen, DSF (Nagel et al. 2015), welcher in Zusammenarbeit mit dem Patienten von den Anästhesieärzten dieser Studie angewandt wurden.

Mithilfe der Schmerzanamnese wurden auch Informationen über den Opioid-, Analgetika- und Koanalgetikaverbrauch des jeweiligen Patienten vor der Operation erhoben. Diese Informationen verwendeten wir für die Errechnung der MQS (Medication Quantification Scale). Wir verwendeten in der Rechnung die Angaben der Gewichtung der Schadenspotenz von Harden et al. 2005, bedienten uns aber der Medikamentenklassen Paracetamol, Cox-2-Hemmer, Trizyklische Antidepressiva, sonstige Analgetika (z. B. Tramadol, Tilidin, Novalgin), Opioide nach Schema II, nichtsteroidale Antirheumatika und Muskelrelaxantien mit Abhängigkeitspotential. Das 
Dosierungslevel von 1, subtherapeutische Dosierung, bis 4, supratherapeutische Dosierung, behielten wir jedoch bei.

Insgesamt berechneten wir 2 verschiedene MQS-Parameter als Summenscore, einen für alle Medikamente ohne Opioide und einen weiteren ausschließlich mit Opioiden.

\subsubsection{Patientenfragebogen CPG}

Der Chronic Pain Grade (von Korff et al. 1992) ist ein auch auf Deutsch validierter Schmerzfragebogen (Nagel et al. 2015), welcher den Schweregrad der Schmerzerkrankung feststellt (Klasen et al. 2004). Nach von Korff werden die Patienten in insgesamt 5 Gruppen eingeteilt. In die erste Gruppe Grad 0 werden Patienten ohne Schmerzen eingeordnet. Die anderen 4 Gruppen sind die Schmerzgruppen. In Grad I sind Patienten mit geringer Beeinträchtigung und geringer Schmerzintensität, Grad II geringe Beeinträchtigung - hohe Schmerzintensität, Grad III hohe Beeinträchtigung moderate Einschränkung und Grad IV hohe Beeinträchtigung - ernsthaft eingeschränkt. Dies wird ermittelt, indem die Schmerzintensität mit 3 Items und die schmerzbezogene Beeinträchtigung mit 4 Items berechnet werden.

Es wird dabei sowohl die Intensität des akuten Schmerzes, als auch der stärkste und durchschnittliche Schmerz in den letzten 3 Monaten, erfragt. Die Patienten können ihre Schmerzen dann auf einem Score von 0 bis 10 angeben, wobei 0 für "kein Schmerz“ und 10 für „stärkster vorstellbarer Schmerz" steht. Die Beeinträchtigung wird ebenfalls rückblickend auf die letzten 3 Monate betrachtet. Dabei können die Patienten sowohl bei einem Item die Anzahl der qualitätsgeminderten Tage, als auch bei den übrigen 3 Items die Beeinträchtigung auf einer Scala von 0 bis 10 angeben, wobei auch hier 0 für „keine Beeinträchtigung“ und 10 für „keine Aktivität mehr“ steht.

Aus den Fragen 1-3 wird ein Maß der Schmerzintensität errechnet. Der Mittelwert dieser drei Fragen wird mit dem Faktor 10 multipliziert, um einen Score von 0-100 erstellen zu können. Um die schmerzbezogene Beeinträchtigung der Patienten identifizieren zu können wird von den Fragen 5-7 ebenfalls der Mittelwert errechnet und mit dem Faktor 10 multipliziert, um den Scorewert von 0-100 bestimmen zu können. Anhand dieses 
Wertes erfolgt in einem weiteren Schritt die Einteilung in 4 Gruppen. Für 0-29 \% gibt es 0 Punkte, 30-49 \% erhalten 1 Punkt, 50-69 \% bekommen 2 Punkte und alles über $70 \%$ erhält 3 Punkte. Des Weiteren werden Punkte für die Arbeitsunfähigkeit anhand der 4. Frage vergeben. Eine Arbeitsunfähigkeit von 0-6 Tagen gaben 0 Punkte, 7-14 Tagen 1 Punkt, 15-30 Tage 2 Punkte und alles über 31 Tage 3 Punkte. Die Punkte der Arbeitsunfähigkeit und schmerzbezogenen Beeinträchtigung ergeben zusammen die Punkte für die Beeinträchtigung.

Zur finalen Einteilung der Grade werden nun die Beeinträchtigungspunkte und der Schmerzintensitätsscore verwendet.

Tabelle 1: CPG-Einteilung

\begin{tabular}{|l|l|l|}
\hline Klassifikation & Schmerzintensität & Beeinträchtigungspunkte \\
\hline Grad 0 & Kein & Kein \\
\hline Grad I & $<50$ & $<3$ Punkte \\
\hline Grad II & $>50$ & $<3$ Punkte \\
\hline Grad III & $>0$ & $3-4$ Punkte \\
\hline Grad IV & $>0$ & $5-6$ Punkte \\
\hline
\end{tabular}

\subsubsection{Patientenfragebogen painDETECT}

Während der Chronic Pain Grade eine Möglichkeit der Unterscheidung zwischen verschiedenen Schweregraden chronischer Schmerzen ermöglicht, kann man mit dem painDETECT (Frevnhagen et al. 2006) neuropathische Schmerzcharakteristika identifizieren und quantifizieren. Ein positiver Score ist nicht mit der Diagnose eines neuropathischen Schmerzsyndroms gleichzusetzen. Die erste Frage des painDETECT bezieht sich auf Schmerzen aktuell, diese wurde bei identischer Formulierung aus dem 
Chronic Pain Grade übernommen. Die zwei weiteren Fragen bezüglich der Dauer der Schmerzen wurden von 4 Wochen auf drei Monate gesetzt.

Diese Umstellung beeinflusst den Score nicht, da sich dieser aus den weiteren 7 Fragen zur Schmerzcharakteristik ergibt, welche von den Patienten mit 6 Antwortmöglichkeiten von "niemals“ bis zu „sehr stark“ beantwortet werden können, und mit einer Punkteskala von 0 bis 5 bewertet werden. Zudem gibt es noch ein Item über die Schmerzausstrahlung, der mit "ja“ oder „nein“ beantwortet werden kann, wobei „ja“ 2 Punkte erhält und „nein“ 0 . Zuletzt gibt es vier Items bezüglich des zeitlichen Schmerzverlaufes, welche unterschiedlich mit Punkten bewertet werden. Ein kontinuierlicher Schmerz mit leichten Schwankungen erhält keine Punkte und mit Schmerzattacken -1. Gibt der Patient ausschließlich Schmerzattacken oder starke Schwankungen im Schmerzverlauf an, so wird das mit +1 Punkt bewertet. Insgesamt können bei diesem Fragebogen maximal 35 Punkte erreicht werden.

Bei einem Score von 0-12 ist ein neuropathischer Schmerz unwahrscheinlich (<10\%), bei 13-18 ist der Score uneindeutig, bei 19-35 ist ein neuropathischer Schmerz wahrscheinlich $(>80 \%)$. Der painDETECT wurde für Schmerzerkranungen mit überwiegend rein neuropathischen Anteil (Postzosterneuralgie und schmerzhafte diabetische Polyneuropathie) validiert. Die Aussagekraft für Schmerzbilder nach Operationen ist vermutlich deutlich geringer.

\subsubsection{Fragebogen MPSS}

Der MPSS (Mainz Pain Staging Score; Gerbershagen 1986) zielt auf die Erfassung einer Schmerzchronfizierung und wurde nicht primär für arthrosebedingten Hüftschmerz entwickelt. Primär wurde der Score für Rückenschmerzpatienten, später aber auch für andere Schmerzgruppen validiert wie Kopfschmerzen, neuropathische und muskuloskeletterale Schmerzen (Frettlöh et al. 2003). Er ist demnach auch bei chronischen Hüftschmerzen sinnvoll. Insgesamt deckt er mit seinen 4 Klassen sowohl den zeitlichen Hintergrund mit 3 Items, und den räumlichen Aspekt mit einem Item ab, als auch den Medikamentengebrauch mit 2 Items und grob die Inanspruchnahme des Gesundheitssystems mit 4 Items. Jedes Item kann dabei einen Punktwert von 1 bis 3 
Punkten erreichen, wobei in jeder Fragenklasse die Punktzahl durch die Anzahl der Items geteilt wird. Folglich erreicht jeder Patient mindestens einen Score von 4 und maximal einen Score von 12 als Maß für das Stadium der Chronifizierung. Mit einem Summenscore von 4-6 Punkten gelangt der Patient in die erste MPSS Gruppe, mit einem Summenscore von 7-8 in die zweite MPSS Gruppe und ab 9 Punkten in die dritte MPSS Gruppe.

\subsubsection{Patientenfragebogen SF12}

Der SF12 (Short Form), eine validierte Kurzfassung mit 12 Fragen aus dem SF 36 (Ware et al. 1996), erfasst die Lebensqualität und somit sowohl die physische als auch die mentale Gesundheit mit 2 verschiedenen Summenscores und wurde auf Deutsch validiert (Bullinger und Kirchberger 1998). Beim SF12 Körper werden 6 Items bezüglich Schmerzen, körperliche Funktion, körperliche Rolle im Leben sowie die generelle Gesundheit erfragt. Der SF12 Psychisch erfragt mit seinen 6 Items die psychische Stellung im Leben, mentale Gesundheit, Lebenskraft und die psychische Funktionalität (Gandek et al. 1998, Bullinger und Kirchberger 1998). Während die einzelnen Items insgesamt einen Scores von 0 bis 100 ergeben, wobei 0 für die schlechtmöglichste Lebensqualität und 100 für die bestmögliche Lebensqualität steht, werden die Summenscores zu einer Bevölkerungsstichprobe in Bezug gesetzt. Dabei entspricht ein Wert von 50 etwa dem der Bevölkerungsstichprobe, jede Abweichung um 10 entspricht in etwa einer Standardabweichung, wobei die Genauigkeit im negativen Bereich größer ist.

\subsubsection{Patientenfragebogen PHQ15}

Der PHQ15 (Patient Health Questionnaire - Gesundheitsfragebogen für Patienten) (Kroenke et al. 2002) hingegen beschäftigt sich mit der Somatisierung und ist ein Ausschnitt des Gesundheitsfragebogen für Patienten (Spitzer et al. 1999). Zwei Fragen, die sich auf die psychische Komponente beziehen, wurden in unserer Studie nicht verwendet. Der PHQ zielt mit den verbleibenden 13 Fragen auf die Beeinträchtigung des Patienten in der letzten Woche durch dessen körperliche Symptome bzw. die Tendenz, körperliche Symptome zu berichten. Eine Frage befasst sich explizit mit 
Menstruationsbeschwerden oder anderen Probleme mit der Menstruation. Diese Frage ist nicht von Männern oder Frauen nach dem Klimakterium beantwortet worden. Bei diesem Fragebogen können die Patienten pro Item zwischen drei Antwortmöglichkeiten, „nicht beeinträchtigt", „wenig beeinträchtigt" bis „stark beeinträchtigt", die Zutreffende auswählen. Für "wenig beeinträchtigt" erhalten die Patienten für das entsprechende Item +1 und für „stark beeinträchtigt" +2. Hinweisend auf eine leichtes somatoformes Syndrom ist ein Wert ab 5, ab 10 spricht man von einem mittleren und ab 15 von einem starken somatoformen Syndrom, wenn keine anderen Ursachen gefunden werden können (Kroenke et al. 2002). Diese Einordnung, die sich auf den PHQ15 bezieht, wurde von uns nicht verwendet, sondern nur der absolute Wert. Um diesen Gesamtscore mit einem Wert nach der Operation zu vergleichen, wurden diese Fragen auch am 7. postoperativen Tag gestellt.

\subsubsection{Patientenfragebogen DASS}

Das psychische Befinden wurde durch verschiedene weitere Fragebögen genauer bestimmt. So bezieht sich der DASS (Lovibond und Lovibond 1995) auf die Depressivität, Ängstlichkeit und Stressbewältigung. Da wir die Kurzform mit 21 Fragen nahmen, wurde jede dieser psychischen Verfassungen mit je 7 Items abgefragt, wobei jeder Item mit 0 Punkten „trifft gar nicht auf mich zu“ bzw. „nie“ bis 3 Punkten „trifft sehr stark auf mich zu“ bzw. „meistens“ bewertet wird. Die Items beziehen sich dabei nur auf die vorherige Woche. Insgesamt können 21 Punkte für jedes psychische Befinden erreicht werden, da die Summe der jeweiligen Themen den zugehörigen Score bilden. Die Cut-Off Werte liegen dort für Depression bei $>10$, für Ängstlichkeit bei $>6$ und für die Stressbewältigung bei $>10$. Der Fragebogen wurde auch auf Deutsch für Schmerzpatienten validiert (Nilges und Essau 2015).

Eine weitere Möglichkeit wäre die psychische Gesamtbelastung mithilfe eines Summenscores zu betrachten. Insgesamt wäre der Summenscore in diesem Fall dann bei maximal 63 Punkten. Allerdings wurde in dieser Doktorarbeit auf die Ausrechnung verzichtet, da explizit die unterschiedlichen psychischen Parameter untersucht wurden. 


\subsubsection{Patientenfragebogen TSK}

Die bewegungsbezogene Angst hingegen wurde mit dem TSK (Tampa scale of Kinesiophobia), welcher von Miller et al. 1991 entwickelt und erstmals von Vlaeyen et al. 1995 veröffentlicht wurde, erfasst. Auch dieser Fragebogen ist in einer deutschen Version validiert (Rusu et al. 2014). Auch dort werden die insgesamt 17 Items mit Punkten von 0 bis 3 bewertet, wobei vier Fragen invers skaliert sind und bei diesen Fragen der Score von 3 bis 0 Punkten gerechnet wird. Insgesamt kann folglich ein Gesamtscore von 21 Punkten erreicht werden.

\subsubsection{Patientenfragebogen KSI}

Das Katastrophisieren, sowie Hilfe und Hoffnungslosigkeit und den jeweiligen Durchhalteappell des Patienten ermittelten wir durch das KSI (Kieler Schmerzinventar) (Hasenbring 1993). Die Antworten werden von 0 "nie" bis 6 "immer" durch eine LikertScale mit 7 Antwortmöglichkeiten je Frage eingeteilt. Dabei wird Katastrophisieren mit insgesamt 5 Items, der Durchhalteappell mit 4 Items und der Zustand von Hilfe und Hoffnungslosigkeit mit 9 Items bestimmt. Der errechneten Summenscore für die einzelnen Bewältigungsstrategien wird dann durch die Anzahl der Fragen geteilt, um einen vergleichbaren Mittelwert bestimmen zu können.

\subsubsection{Patientenfragebogen WOMAC}

Der WOMAC (Bellamy et al. 1988) hingegen ist ein hüftspezifischer Outcomefragbogen der auf der Selbsteinschätzung durch den Patienten beruht. Der Fragebogen bezieht sich die subjektive Beweglichkeit mit 17 Items, Steifigkeit mit 2 Items und Schmerzen bei bestimmten Bewegungen mit 5 Items. Dabei benutzt der Fragebogen die Likert-Skala mit je elf Antwortmöglichkeiten zur Beeinträchtigung, wobei 0 für „kein“ und 10 für „extrem" steht. Verändert wurde dieser Fragebogen auch im Verlauf am 7. postoperativen Tag erfasst, um Veränderungen durch die Operation zu erkennen, welche aber in dieser Doktorarbeit nicht beschrieben werden. 


\subsubsection{Patientenfragebogen QUIPS}

Nach der Operation und während des stationären Aufenthaltes bestimmten wir am 1., 3., 5., und zuletzt am 7. postoperativen Tag mithilfe des QUIPS-Fragebogen (Qualitätsverbesserung in der postoperativen Schmerztherapie) (Meissner et al. 2006) den Schmerzintensitätsverlauf und das subjektive Empfinden der Patienten. Eigentlich entwickelt, um die postoperative Akutbehandlung in teilnehmenden Krankenhäusern einem ständigen Qualitätsmanagement zu unterziehen im Sinne eines BenchmarkProjekts, erfragt er sowohl den Belastungsschmerz als auch den stärksten und den geringsten Schmerz seit der Operation auf einer Scala von 0 bis 10, wobei 0 wie beim Chronic Pain Grade für „kein Schmerz“ und 10 für „stärkster vorstellbarer Schmerz" steht. Weitere 4 Fragen beziehen sich speziell auf die Beeinträchtigung durch Schmerzen bei Bewegung, Husten, Schlaf und Stimmung, 3 Fragen speziell auf mögliche Nebenwirkungen wie Müdigkeit, Übelkeit und Erbrechen, welche vom Patienten mit ,ja“ oder „nein“ beantwortet werden. Zudem wird der Patient gefragt, ob er sich ausreichend über die Schmerztherapie aufgeklärt fühlt, ob er mehr Schmerzmittel gewünscht hätte und ob er mit der Schmerzbehandlung seit der Operation auf einer Scala von 0 bis 15 zufrieden ist. Dabei steht 0 für „völlig unzufrieden“ und 15 „völlig zufrieden“. (Meissner et al. 2008) Da der QUIPS-Fragebogen speziell auf den ersten postoperativen Tag ausgerichtet ist und sich die Fragen auf die Periode der letzten $24 \mathrm{~h}$ beziehen, haben wir an den folgenden Tagen eine angepasste Version des QUIPS verwendet bei Erhalt des Zeitbezugs.

\subsubsection{Mobilität}

Neben den Fragebögen mit Selbstangaben des Patienten wurde zur weiteren objektivierbaren Beurteilung der Mobilität und Balance präoperativ auch ein standardisierter Timed „Up and Go"-Test sowie die Treppengehfähigkeit für die körperliche Aktivität gegen die Schwerkraft geprüft.

Beim Timed „Up and Go“-Test, ursprünglich ein Test von Mathias et al. 1986, entwickelt und später modifiziert, mussten die Patienten sich von einem Stuhl mit Armlehne auf Kommando erheben, 3m bis zu einem markierten Punkt in einem sicheren Gang laufen, 
an diesem Punkt eine $180^{\circ}$-Wendung durchführen und zurück zum Stuhl gehen, um sich auf diesen wieder zu setzen. Erst wenn die Patienten wieder Platz genommen hatten, wurde die Zeit gestoppt. Wenn der Patient in seinem Altag eine Gehhilfe benutzte, so sollte diese auch bei dem Test benutzt werden. Dieser Test ergibt kein valides aber grobes Maß für Balance und Mobilität der Patienten. Die Patienten wurden in der Auswertung anschließend in 5 Gruppen eingeteilt. In die erste Gruppe gelangten die Patienten, wenn sie diese Aufgabe unter $10 \mathrm{~s}$ bewältigen konnten, in die Zweite, wenn sie zwischen 10 und $<20$ s brauchten und in die dritte, wenn sie zwischen 20 und $<30$ s für diese Aufgabe benötigten. Alle weiteren Patienten wurden in die 4. Gruppe eingeteilt, wenn sie die Aufgabe grundsätzlich bewältigen konnten. Patienten, welche die Aufgabe nicht schafften, wurden in die 5. Gruppe eingeteilt (Podsiadlo und Richardson 1991).

Auch beim Treppengehversuch wurde die Zeit gemessen und die erreichten Stufen gezählt. Die Patienten hatten die Aufgabe, 10 Stufen am Stück im Wechselschritt ohne Pause zügig zu bewältigen. Dabei durften Gehhilfen verwendet werden, wenn der Patient diese auch im Altag beim Treppensteigen benutzte. Die Patienten wurden in der Auswertung anschließend in 5 Gruppen eingeteilt. In die erste Gruppe gelangten jene Patienten, die unter $10 \mathrm{~s}$ benötigten. In die zweite Gruppe jene Patienten, die von 10 bis $<20 \mathrm{~s}$, in die 3. Gruppe Patienten, die von 20 bis $<30 \mathrm{~s}$ und in der 4. Gruppe Patienten, die größer gleich $30 \mathrm{~s}$ benötigten. In die 5 . Gruppe wurden die Patienten eingeteilt, welche die Stufen nicht am Stück bewältigen konnten, eine Pause benötigten oder den zweiten Fuß auf eine bereits erreichte Stufe nachziehen mussten, um die Treppe zu bewältigen (Zeni et al. 2014).

\subsubsection{Physiologische Schmerzmessung}

Sowohl präoperativ als auch am 3. postoperativen Tag wurde eine Schmerzschwellenmessung an 10 fest definierten Stellen des Körpers (bilateral auf dem Daumennagel, am laterale Epicondylus, Musculus trapezius, Musculus quadriceps femoris sowie Musculus tibialis anterior) mit einem Druckalgometer (Hersteller: Wagner Instruments, Greenwich, CT 06836-1217, USA, CE-Zertifizierung vorhanden, Einsatz in der Studie gemäß der vorgesehenen und zertifizierten Anwendung) durchgeführt. Die Patienten wurden vor der Messung über den Messablauf informiert und ein Testdurchlauf durchgeführt. Bei der 
Messung wurde das Algometer senkrecht zur Haut platziert und der Druck linear mit etwa einer Geschwindigkeit von $50 \mathrm{kPa} / \mathrm{s}$ gesteigert. Die Patienten wurden instruiert, wenn der zunehmende Druck zum Schmerz wurde, die Messung mit "STOP“ abzubrechen. Der entsprechende Wert wurde als Druckschmerzschwelle notiert. Die Reihenfolge der Messungen war für alle Patienten identisch, an jedem Messort wurde nur eine Messung durchgeführt. Für die vorliegende Studie wurde der Mittelwert über alle 10 Messpunkte als Maß für die generelle Druckschmerzempfindlichkeit verwendet.

\subsubsection{Routinedaten}

Um herauszufinden, welche Medikamente die Patienten in welcher Dosierung und zu welchem Zeitpunkt erhalten haben und welche Besonderheiten es gab, wurden diese Informationen aus den Patientenakten gesammelt. Dort konnte auch die Länge des Aufenthaltes auf der Intensivstation oder im Aufwachraum sowie die Informationen bezüglich der Operation selbst, wie beispielsweise Dauer der Operation, anhand der Schnitt-Naht Zeit als Maß des operativen Traumas, und verwendete Narkoseart, entnommen werden.

\subsubsection{Präoperatives Morphinäquivalent}

Um die eingenommenen Opiate präoperativ vergleichen zu können, wurden sie in die Äquivalentdosis oraler Morphine umgerechnet. Oxycodon hat dabei einen Umrechnungsfaktor von 0,75, Hydromorphon 0,13, Piritramid 1,5, Fentanyl 0,01, Tilidin und Tramadol 10, Buprenorphin 0,03 und intravenöses Morphin 1/3.

Die weiteren Umrechnungen in die MQS (Medication Quantification Scale) wurden bereits zuvor in dieser Arbeit beschrieben.

\subsection{Narkose- und Analgetikaverbrauch}

In diese Doktorarbeit wurden Patienten eingeschlossen, welche eine Vollnarkose erhielten. Diese bestand aus einem standardisierten Verfahren. Zunächst erhielten die Probanden als Prämedikation am Vorabend und am Tag der Operation Clorazepat 20$30 \mathrm{mg}$. 
Zur Einleitung wurde Remifentanil (1-1,5 $\mu \mathrm{g} / \mathrm{kgKG}$ (Körpergewicht)) und Propofol (1-2 $\mathrm{mg} / \mathrm{kgKG}$ ) für 3 Minuten intravenös verabreicht. Als Muskelrelaxanz zur oralen Intubation wurde Atracurium (0,5 mg/kgKG) verwendet.

Zur Aufrechterhaltung der Narkose wurde Sevofluran 0,7-1,0 inhalativ oder Propofol intravenös (3,5-4,5 mg/kgKG pro Stunde) sowie zur Analgesie Remifentanil (0,15-0,25 $\mu \mathrm{g} / \mathrm{kg} / \mathrm{min}$ ) verwendet.

Während des Einbringens der Endoprothese erhielten die Probanden 0,1 mg/kgKG Piritramid sowie $15 \mathrm{mg} / \mathrm{kgKG}$ Metamizol bzw. die vergleichbare Dosis Paracetamol bei Unverträglichkeit.

Im Aufwachraum erhielten die Probanden je nach Alter und Gewicht 10-20 mg retardiertes Oxycodon, sowie $600 \mathrm{mg}$ Ibuprofen oral (Abbildung 1) (Erlenwein et al. 2016). 


\section{Basisschmerzmedikation}

1. $3 \times 600 \mathrm{mg}$ Ibuprofen oder $4 \times 1 \mathrm{~g}$ Novalgin oder $4 \times 1 \mathrm{~g}$ Paracetamo

2. retardiertes Oxycodon $2 \times 10-20 \mathrm{mg}$

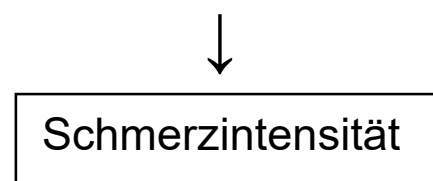

$\downarrow$

NRS > 3 über 60 min

$\max .3 x$

Gabe von Hydromorphon 1,3-2,6 mg

Nach 60 min Frage nach

Schmerzintensität
NRS $\leq 3$ über $24 \mathrm{~h}$

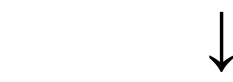

Reduktion der

Basisschmerzmedikation

nach der 3. Gabe $\downarrow$

Arzt hinzuziehen

1. Evaluation Ursache des Schmerzes und ggf. Intervention

2. Erhöhung der

Basisschmerzmedikation

\section{Abbildung 1: Schmerzmedikationsschema Hannover}

Im stationären Verlauf erhielten die Probanden 2 Mal täglich retardiertes Oxycodon äquivalent zu der ermittelten Dosis im Aufwachraum und 3 Mal täglich Ibuprofen. Die Probanden wurden von dem pflegerischen Personal 3 Mal täglich nach den Schmerzen gefragt. War der NRS $<3$ wurde nach 24 Stunden die Opioiddosis verringert. Gaben die Probanden Schmerzen $\geq 3$ an, so wurde innen 1,3-2,6 mg Hydromorphon angeboten. Waren die Schmerzen 60 min nach der Einnahme weiterhin $\geq 3$, so wurde innen erneut Hydromorphon angeboten und das Procedere wiederholt. Gaben die Patienten beim 
dritten Mal wieder Schmerzen auf der NRS $\geq 3$ an, so konnten sie erneut Hydromorphon bekommen und der Stationsarzt wurde hinzugezogen, um mögliche Komplikationen wie beispielsweise eine tiefe Beinvenenthrombose auszuschließen. Des Weiteren wurde nach der dritten Einnahme des Hydromorphons innerhalb $24 \mathrm{~h}$ die Opioidgabe um 10mg retardiertes Oxycodon (zweimal täglich) erhöht.

\subsection{Statistische Analysen}

Die folgenden Rechnungen wurden in Zusammenarbeit der Beratung der Universitätsstatistik durchgeführt.

Nachdem alle Daten zunächst in Excel erfasst worden waren, wurden mithilfe von STATISTICA 10 die einzelnen Tests und Scores ausgewertet.

Insgesamt wurden 25 Variablen deskriptiv u. a. mit dem Kolmogorov-SmirnovAnpassungstest (K.S.-Test) betrachtet und auf eine Normalverteilung getestet. Die in Frage kommenden Variablen wurden im Rahmen der univariater Regressionen auf Signifikanz geprüft. Da die analoge Schmerzskala von 0 bis 10 geht, wurde für die Endpunkte Schmerz eine ordinale logit Regression durchgeführt. Für den Analgetikagebrauch hingegen verwendeten wir eine lineare Regression.

Alle signifikanten Variablen der univariaten Analyse (mit $p<0,05$ ) wurden dann in einem darauffolgenden Schritt einer multiplen Regression unterzogen. Alle 25 Variablen bei der gegebenen Stichprobegröße gemeinsam in die Analyse einzuschließen, führt zu einem instabilen Modell und birgt die Gefahr, die Ergebnisse stark zu verfälschen. Wir verwendeten eine rückwärts läufige (backward) Regression, um die am stärksten erklärenden Variablen bzw. die am stärksten erklärende Variable identifizieren zu können.

multiple Regression: $Y=\alpha+\beta_{1}{ }^{*} X_{1}+\beta_{2}{ }^{*} X_{2}+\beta_{3}{ }^{*} X_{3}+\beta_{4}{ }^{*} X_{4}+\beta_{5}{ }^{*} X_{5}+\ldots+\beta_{i}^{*} X_{i}+\varepsilon$.

Auch wurde der Versuch unternommen, geschlechtsspezifische Unterschiede zu detektieren. Bei normalverteilten Variablen wurden mit dem t-Test Männer und Frauen verglichen, bei allen nicht-normalverteilten Parametern wurde statt der Regressionsverfahren der Mann-Whitney-U Test eingesetzt. 


\section{Ergebnisse}

\subsection{Charakteristiken des Patientenkollektivs}

\subsubsection{Einschlüsse in das Patientenkollektiv}

Insgesamt waren es 171 Patienten, die sich einem primären endoprothetischen Hüftgelenkersatzes unterzogen. Davon wurden insgesamt 21 nicht in die Studie eingeschlossen, da sie die Einschlusskriterien nicht erfüllten. 11 wollten nicht teilnehmen, 3 nahmen an einer anderen Studie teil, 1 war unter 18 Lebensjahren, 1 war der deutschen Sprache in Wort und Schrift nicht ausreichend mächtig, 3 wechselten während der Datenerhebung das Krankenhaus, 1 war medikamentenabhängig, 1 litt unter Demenz. So blieben 151 Patienten, welche die Studie während ihres Klinikaufenthaltes gänzlich mitmachten und auch allen Einschlusskriterien entsprachen.

Des Weiteren wurden für die endgültige Auswertung alle Patienten aus der statistischen Analyse herausgenommen, die eine Spinalanästhesie erhalten haben, da sich dort der Opioidverbrauch deutlich von anderen Narkoseverfahren unterschied, diese Gruppe aber leider zu klein ist, um statistisch wertvolle Daten herausziehen zu können. Insgesamt waren dies 8 Patienten.

Damit sich der Datensatz von den anderen Arbeiten bzw. Publikationen aus der vorliegenden Studie nicht unterscheidet, wurden weitere 18 Personen aufgrund ihrer besonderen präoperativen Schmerzbeschreibung bezüglich der Schmerzausstrahlung bei akuter Femurkopfnekrose oder postoperativen Komplikationen wie einem Delir nicht in dem finalen Datensatz miteinbezogen (Abbildung 2). 


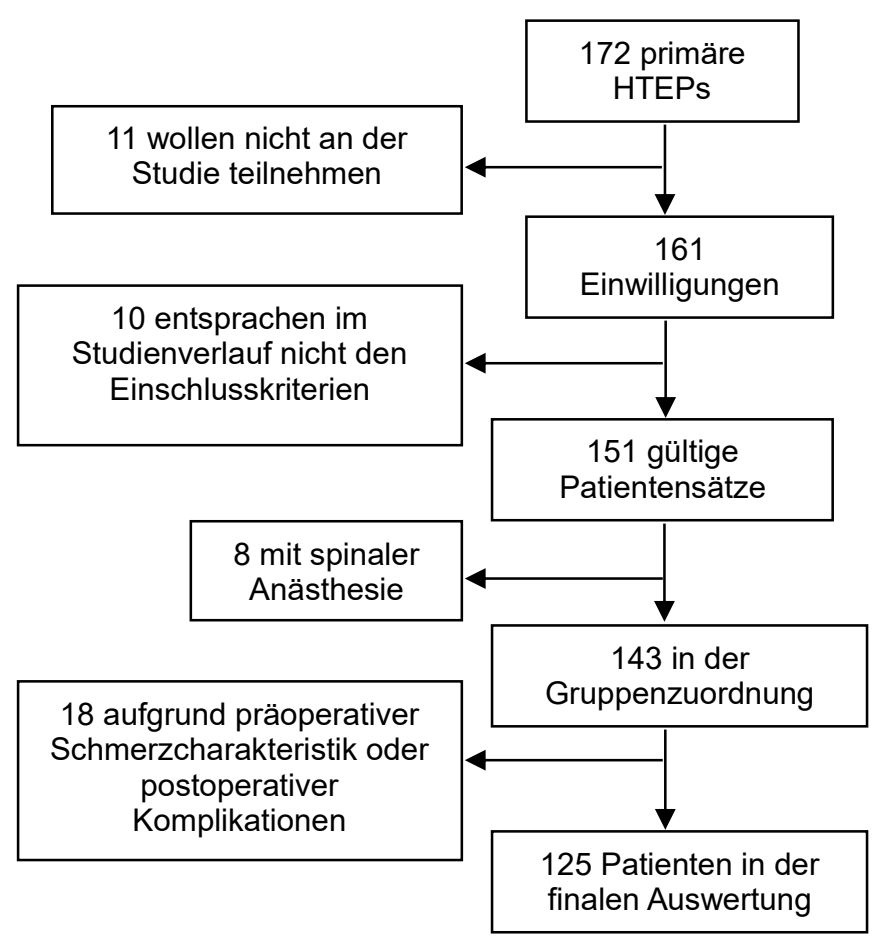

Abbildung 2: Flowchart Patientenkollektiv

\subsubsection{Alter}

Unser Patientenkollektiv von 125 Patienten erstreckt sich über eine Altersspanne von 24 Jahren bis 88 Jahren.

Wie man anhand des Histogramms für das Alter sehen kann (Abbildung 3), erkennt man eine breite Streuung, welche ihren Mittelwert von 63,2 hat. Die Standardabweichung beträgt 12,7. Mit einem p-Wert $>0,2 \mathrm{im}$ K.S - Test handelt es sich um eine normalverteilte Variable. 


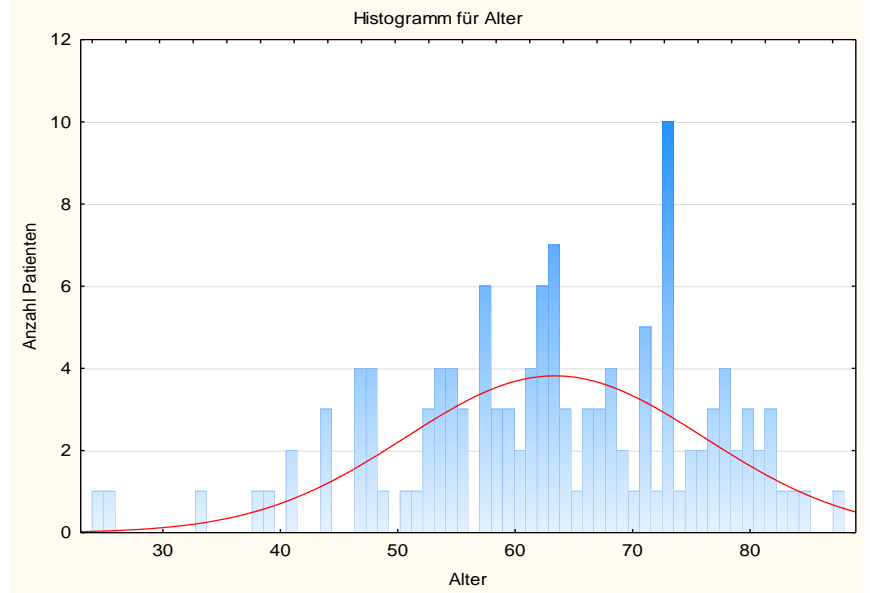

Abbildung 3: Histogramm Alter

\subsubsection{BMI}

Der BMI ist eine Maßangabe, welche die Relation des Körpergewichtes mit der Körpergröße darstellt.

BMI $=\frac{\text { Körpergewicht }(\mathrm{kg})}{\text { Körpergröße }{ }^{*} \text { Körpergröße }\left(\mathrm{m}^{2}\right)}$

Der BMI hat bei unserem Patientenkollektiv eine Spanne von 18,9 bis 45,6. Der Mittelwert beträgt 28,1 mit einer Standardabweichung von 5,4. Laut dem K.S.-Test mit einem $p$-Wert $>0,2$ handelt es sich um eine normalverteilte Variable, was man auch graphisch in Abbildung 4 sehen kann. Interessant dabei ist, dass sich die weibliche Spannweite von 19,1 bis 45,6 mit einem Mittelwert von 28,6 erstreckt und die männliche nur von 18,9 bis 34,1 mit einem Mittelwert von 27,4. 


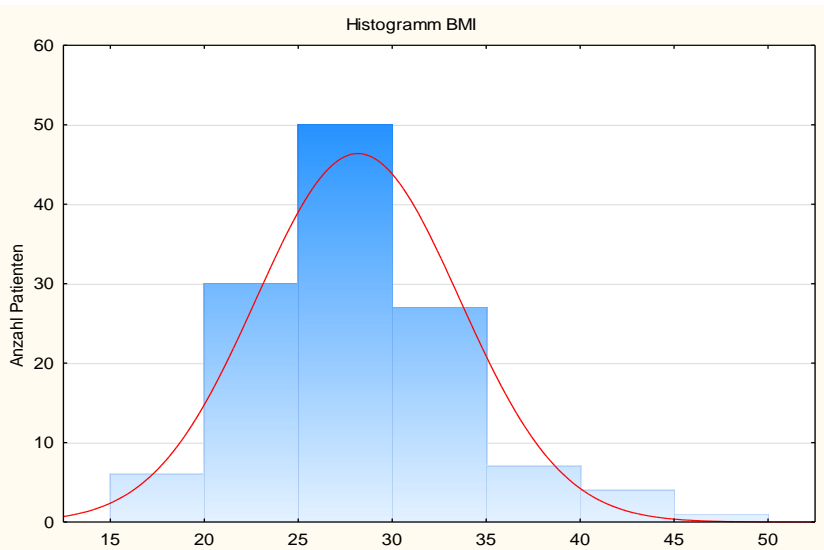

Abbildung 4: Histogramm BMI

Laut Adipositas-Klassifikation der Weltgesundheitsorganisation spricht man von einem Untergewicht mit einem BMI <18,5 und von einem Übergewicht ab einem BMI von 25. Das Übergewicht ist ab einem Wert von 30 behandlungsbedürftig. In diesem Fall spricht man auch von Adipositas. Wird die 40 beim BMI überschritten, so spricht man von Adipositas per magna. Diese findet sich im Patientenkollektiv ausschließlich bei insgesamt 5 weiblichen Teilnehmern.

\subsection{Beschreibung der präoperativ erhobenen Einflussfaktoren}

\subsubsection{DASS}

Wie man an der Verteilung des Patientenkollektivs erkennt, ist zwar eine breite Verteilung beim Parameter DASS-Depression vorhanden (Abbildung 5), aber diese ist nur durch vereinzelte Patienten entstanden. Fast die Hälfte der Patienten $(n=52)$ gibt einen Score von 0 an. Nur 7 Patienten überschritten den Cut-Off-Wert von $>10$. Es handelt sich nicht um eine normalverteilte Variable, was man zusätzlich am K.S.-Test sehen kann, wo der $p$-Wert $<0,01$ ist. 


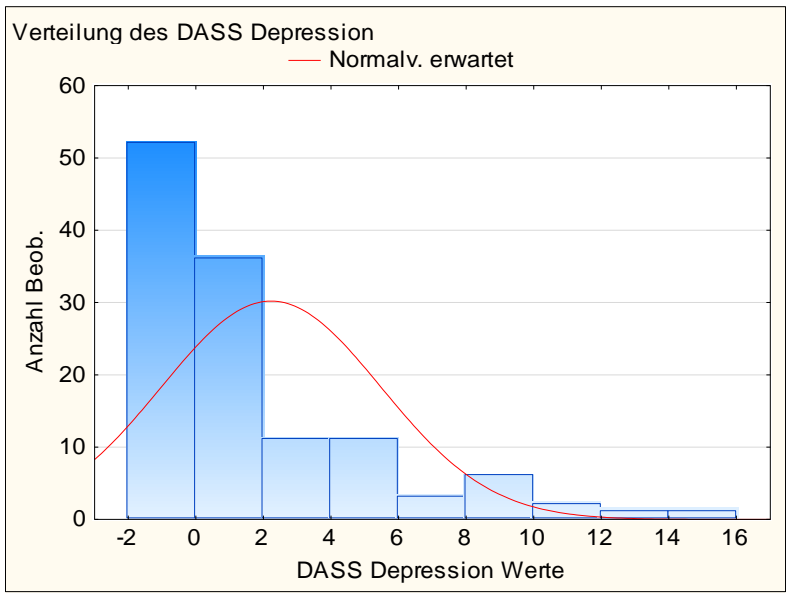

Abbildung 5: Histogramm DASS-Depression

Auch beim DASS-Angst gab es nur eine kleine Patientengruppe von $n=10$, die den Cut off-Wert von $\geq 6$ erreichten. Auch hier gab fast die Hälfte der Patienten, $n=49$, einen Score von 0 an, und man kann entsprechend keine Normalverteilung der Werte identifizieren, was man in Abbildung 6 sieht und der $p$-Wert von >0,01 beim K.S.-Test bestätigt.

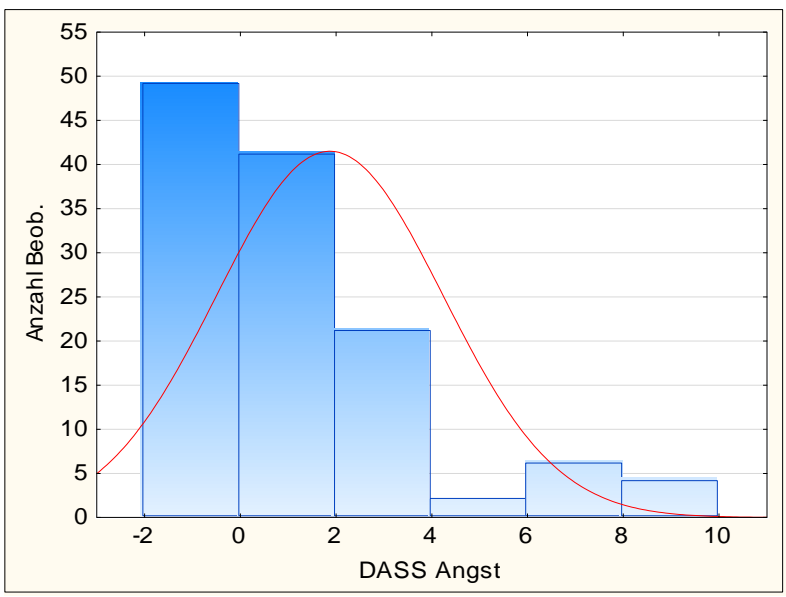

Abbildung 6: Histogramm DASS-Angst 
Betrachtet man jedoch den DASS-Stress, so erreichen insgesamt 20 Patienten den Cut off Wert von $\geq 10$ und 103 Patienten bleiben darunter. Entsprechend ist auch die Verteilung gleichmäßiger und statistisch besser verwertbar (Abbildung 7), auch wenn es sich noch nicht um eine Normalverteilung handelt, da der p-Wert im K.S Test $<0,05$ ist.

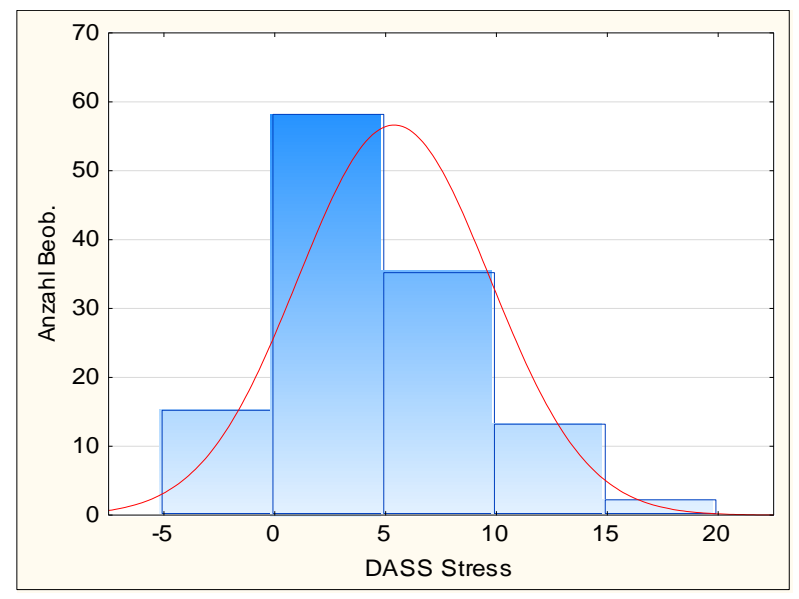

Abbildung 7: Histogramm DASS-Stress

\section{$3.2 .2 \mathrm{KSI}$}

Der KSI für Hilfe und Hoffnungslosigkeit konnte nur bei 106 Patienten ausgewertet werden. Der Mittelwert beträgt 2,1 mit einer Standardabweichung von 1,6. Es handelt sich um eine normalverteilte Variable mit einem $p$-Wert im K.S.-Test von $>0,2$, was man auch graphisch erkennen kann (Abbildung 8) 


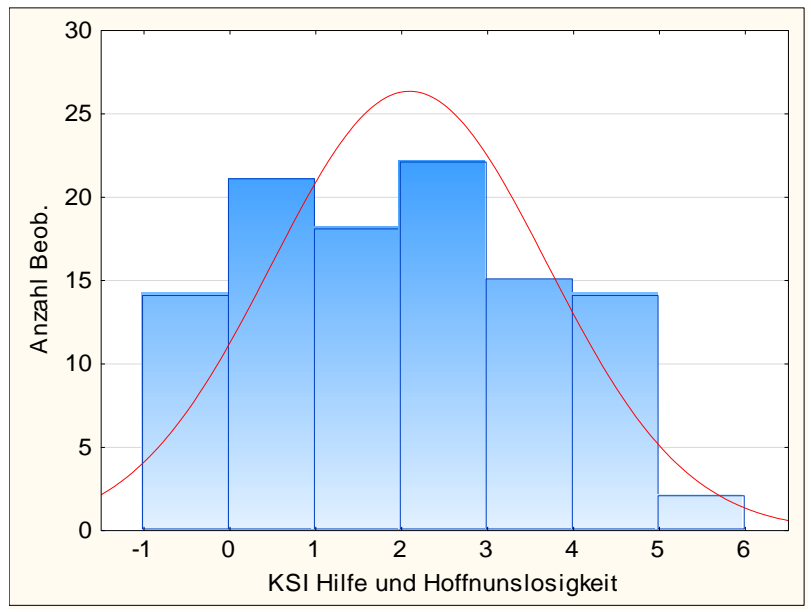

Abbildung 8: Histogramm KSI Hilfe und Hoffnungslosigkeit

Der KSI Katastrophisieren wurde von 108 Patienten vollständig ausgefüllt und ist sowohl graphisch in Abbildung 9 erkennbar als auch statistisch mit einem $p$-Wert von $<0,01 \mathrm{im}$ K.S.-Test nicht normalverteilt. Hier beträgt der Mittelwert 0,9 mit einer Standardabweichung von 1,2 .

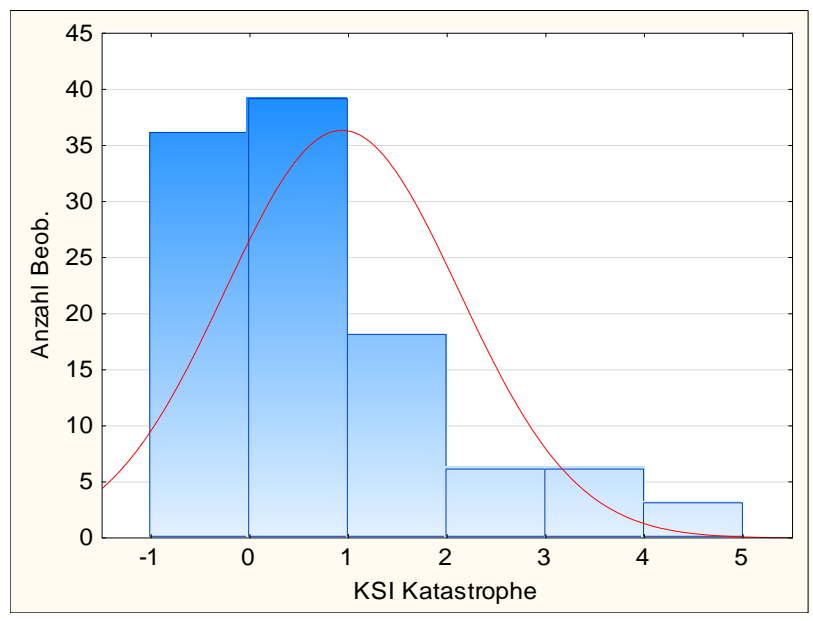

Abbildung 9: Histogramm KSI Katastrophe

Der KSI Durchhalteappell konnte von 111 Patienten verwendet werden. Der Mittelwert ist 2,6 mit einer Standardabweichung von 1,6. Diese Variable ist normalverteilt, was man an dem $\mathrm{p}$-Wert von >0,2 im K.S.-Test erkennen kann (Abbildung 10). 


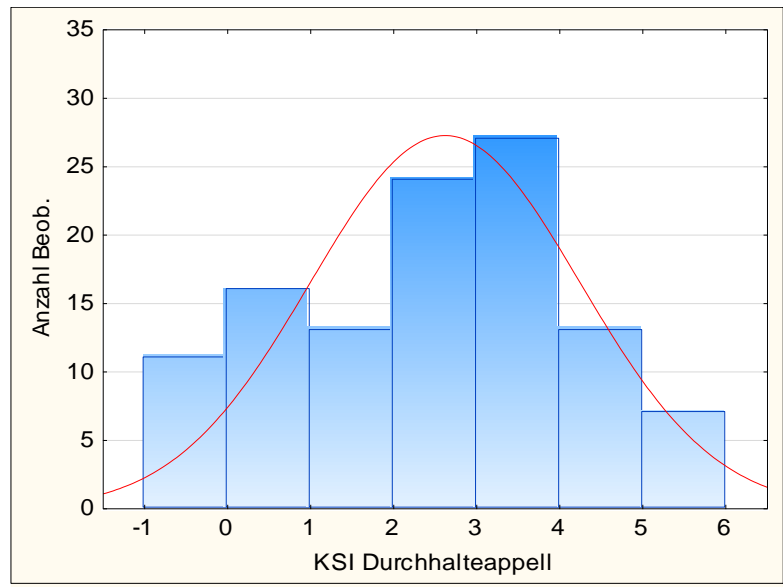

Abbildung 10: Histogramm KSI Durchhalteappell

\subsubsection{TSK}

Der TSK wurde von 108 Patienten vollständig ausgefüllt. Der Mittelwert ist 36,4 mit einer Standardabweichung von 6,3. Der Parameter ist nomalverteilt, was man anhand des $p$ Wertes von >0,2 im K.S.-Test erkennen kann (Abbildung 11).

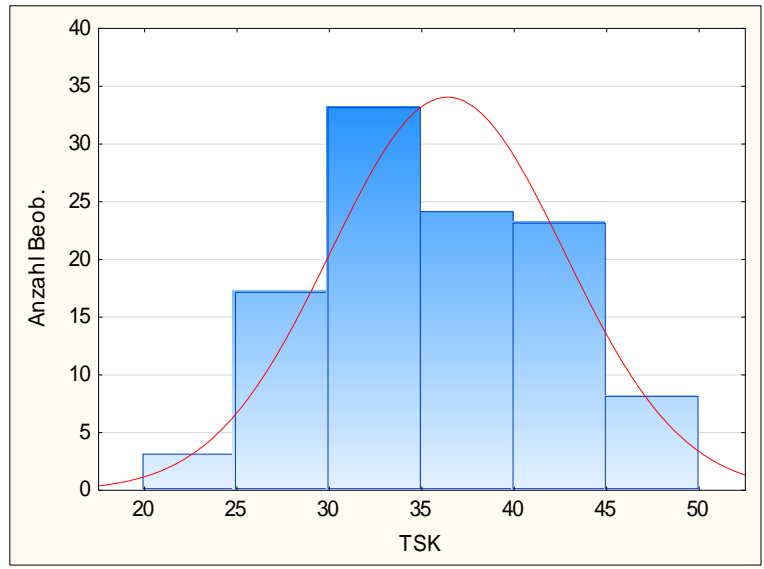

Abbildung 11: Histogramm TSK 


\subsubsection{SF12 Körperliche Summenskala}

Bei 115 Patienten konnte die körperliche Summenskala ausgewertet werden. Der Mittelwert beträgt 30,0 mit einer Standardabweichung von 7,4. Insgesamt ist der Parameter normalverteilt, was man in Abbildung 12 sieht. Der p-Wert im K.S.-Test beträgt $>0,2$. Dies entspricht einer Abweichung zu schlechteren Werten um ca. zwei Standardabweichungen vom Mittelwert der gesunden Normalbevölkerung.

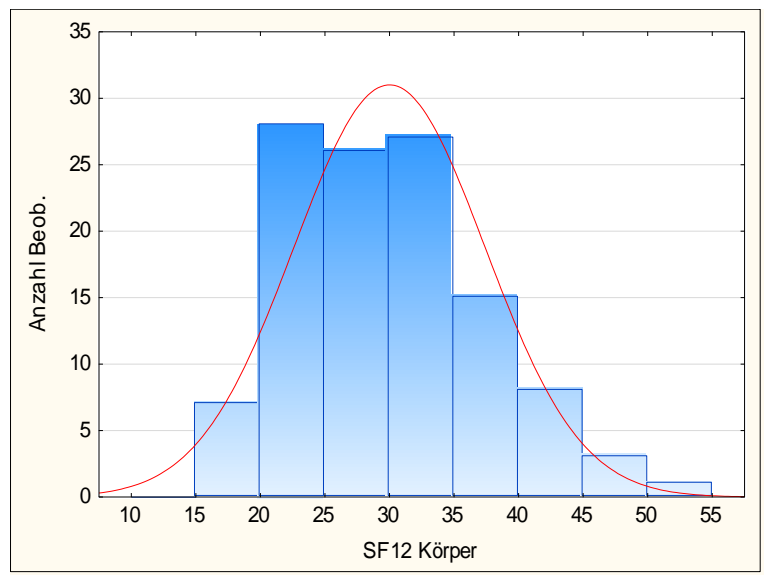

Abbildung 12: Histogramm SF12 Körperliche Summenskala

\subsubsection{SF12 Psychische Summenskala}

Insgesamt konnten für 115 Patienten die psychische Summenskala berechnet werden. Der Mittelwert beträgt 49,3 mit einer Standardabweichung von 11,8. Es handelt sich mathematisch um eine normalverteilte Variable mit einem $\mathrm{p}$-Wert von $>0,2$ im K.S.-Test. Graphisch ist in Abbildung 13 eine Verschiebung in den höheren Zahlenbereich zu erkennen. Psychisch zeigt sich somit im Mittel kein relevanter Unterschied zur Normalbevölkerung. 


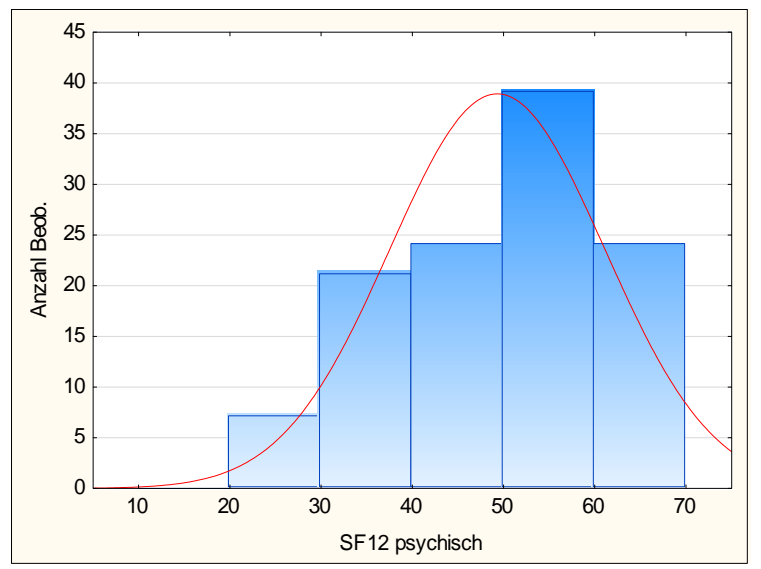

Abbildung 13: Histogramm SF12 psychische Summenscala

\subsubsection{PHQ}

Der PHQ wurde von 109 Patienten vollständig ausgefüllt. Da der K.S.-Test einen p-Wert $>0,1$ hat und man graphisch in Abbildung 14 keine charakteristische Verteilung erkennen kann, ist der Parameter nicht normalverteilt. Der Mittelwert beträgt 6 mit einer Standardabweichung von 3,4.

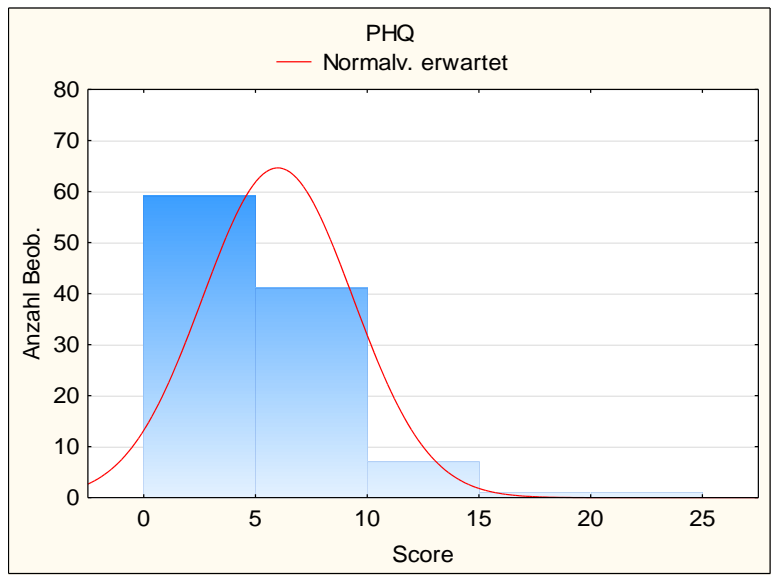

Abbildung 14: Histogramm PHQ15 


\subsubsection{WOMAC}

Insgesamt konnte man man den WOMAC Schmerz Score bei 108 Patienten auswerten. Dieser Parameter ist mit einem p-Wert von $>0,2$ im K.S.-Test normalverteilt, was man auch graphisch in der Abbildung 15 erkennen kann. Der Mittelwert beträgt 51,1 mit einer Standardabweichung von 20,97.

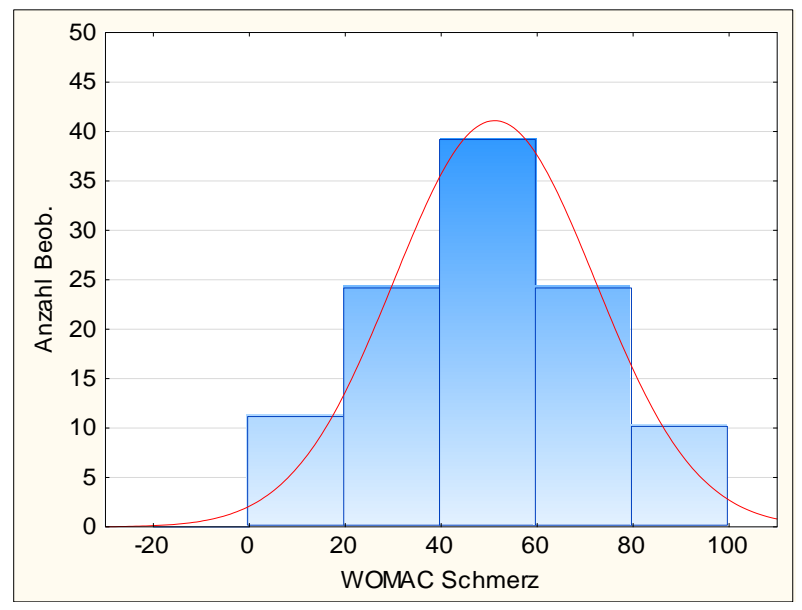

Abbildung 15: Histogramm WOMAC-Schmerz

Beim WOMAC-Steifigkeit konnten 107 Patienten ausgewertet werden. Die Werte sind normalverteilt, da der $p$-Wert im K.S.-Test $>0,2$ ist und man graphisch eine Verteilung entlang der Normalverteilung erkennen kann (Abbildung 16). Der Mittelwert ist 53,9 mit einer Standardabweichung von 13,3.

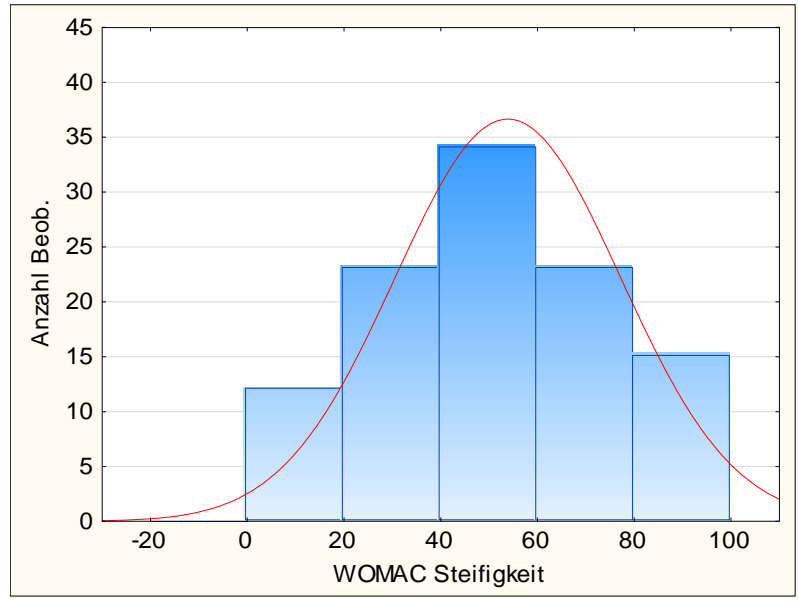

Abbildung 16: Histogramm WOMAC-Steifigkeit 
Betrachtet man den WOMAC Aktivität so handelt es sich auch bei diesem Score um eine graphisch (Abbildung 17) und statistisch normalverteilte Variable mit einem $p$-Wert $>0,2$ im K.S.-Test. Der Mittelwert beträgt 53,9 mit einer Standardabweichung von 21,3.

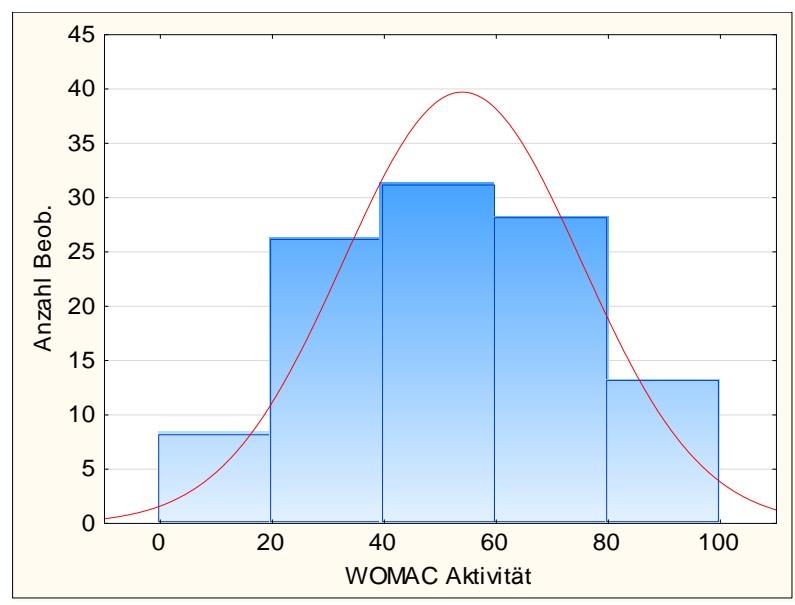

Abbildung 17: Histogramm WOMAC-Aktivität

\subsubsection{Schmerzschwelle}

Bei 122 Patienten konnte die Schmerzschwelle (Abbildung 18) gemessen werden. Der Mittelwert ist 377,9 kPa mit einer Standardabweichung von 179,4 kPa. Dieser Parameter ist normalverteilt, da der $p$-Wert im K.S.-Test $>0,2$ ist und auch graphisch eine breite Streuung erkennbar ist.

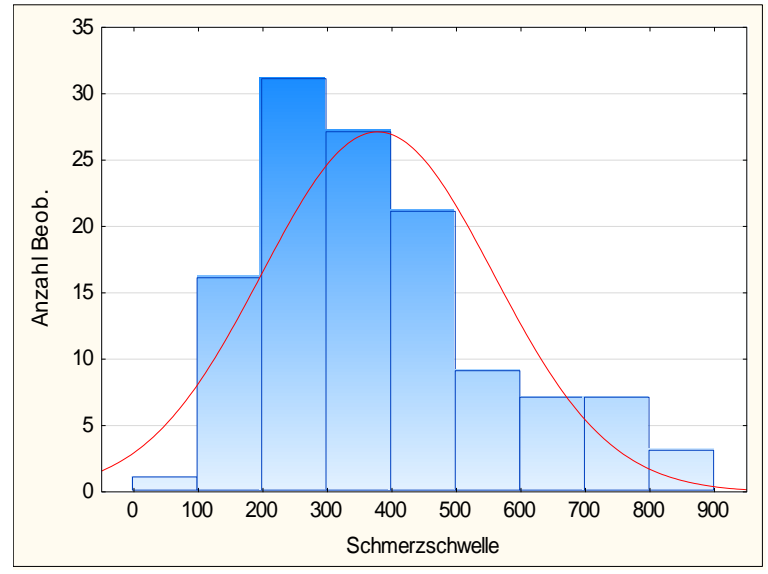

Abbildung 18: Histogramm Schmerzschwelle 


\subsubsection{Funktionsparameter}

Der Treppengehversuch konnte bei insgesamt 120 Patienten untersucht werden und in 5 Gruppen eingeteilt werden. Aus Abbildung 19 ist erkenntlich, dass es die meisten Patienten ( $n=82$, Gruppe 1) geschafft haben 10 Stufen zügig im Wechselschritt am Stück zu gehen und nur 17 Patienten (Gruppe 5), also 14,2 \%, die Aufgabe nicht schafften.

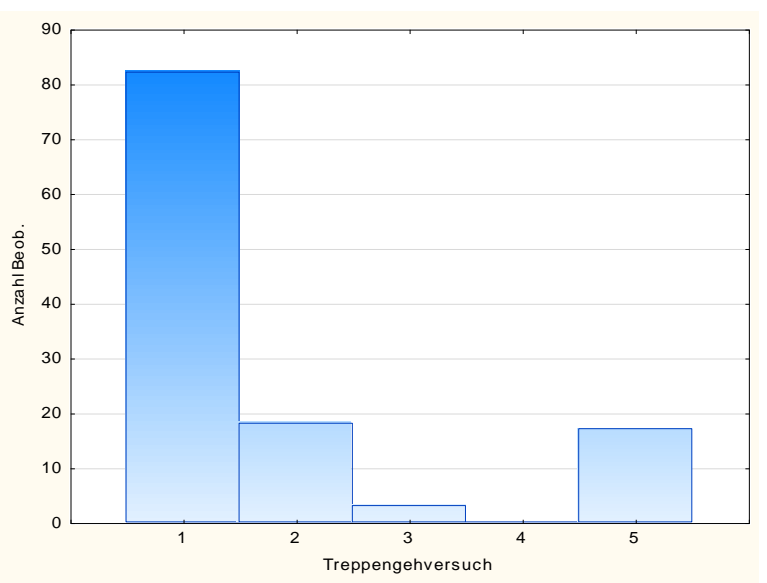

Abbildung 19: Histogramm Treppengehversuch

Der Timed „Up and Go“-Test hingegen konnte bei 119 Patienten ausgewertet werden. Alle Patienten konnten die Aufgabe erfolgreich beenden, wobei in der Abbildung 20 auffällig ist, dass der Hauptanteil der Patienten (Gruppe 2) von 63 \% zwischen 11 und 20 s benötigt hat.

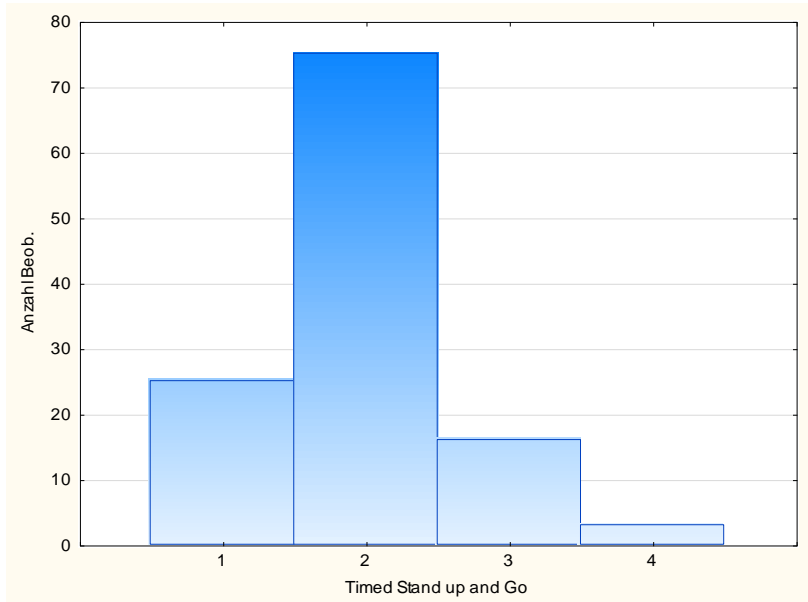

Abbildung 20: Histogramm Timed "Up and Go"-Test 


\subsubsection{0 painDETECT}

Bei 115 Patienten konnte der painDETECT ausgewertet werden. Der Mittelwert beträgt in diesem Kollektiv 9,6 mit einer Standardabweichung von 5,5. Die Werte sind normalverteilt, was man sowohl in Abbildung 21 sieht, als auch an dem K.S.-Test mit einem $p$-Wert >0,2 erkennbar ist. Insgesamt erreichen nur 2 Patienten einen Summenscore über 19, womit die Schmerzcharistika zu $>80 \%$ als neuropathisch ausgewertet werden kann.

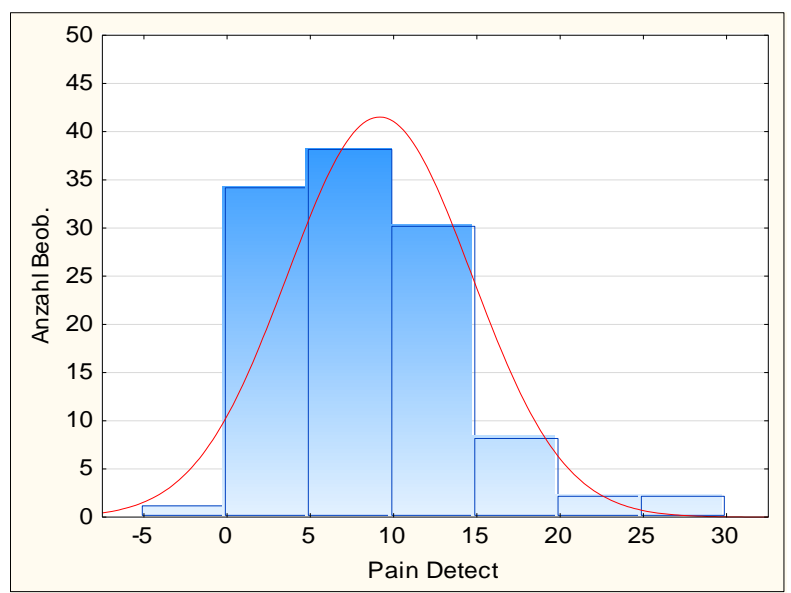

Abbildung 21: Histogramm painDETECT

\subsubsection{MQS Vorbehandlung mit Opioiden und Nichtopioiden}

Die MQS „Vorbehandlung mit Opioiden“ konnte bei allen 125 Patienten errechnet werden. Insgesamt haben 102 Probanden keine Opiode vor der Operation eingenommen. Es handelt sich um einen nicht normalverteilten Parameter, was man graphisch in Abbildung 22 und statistisch am K.S.-Test mit einem p-Wert <0,01 erkennen kann. Der Mittelwert dieses Parameters beträgt 0,6 mit einer Standardabweichung von 1,7. 


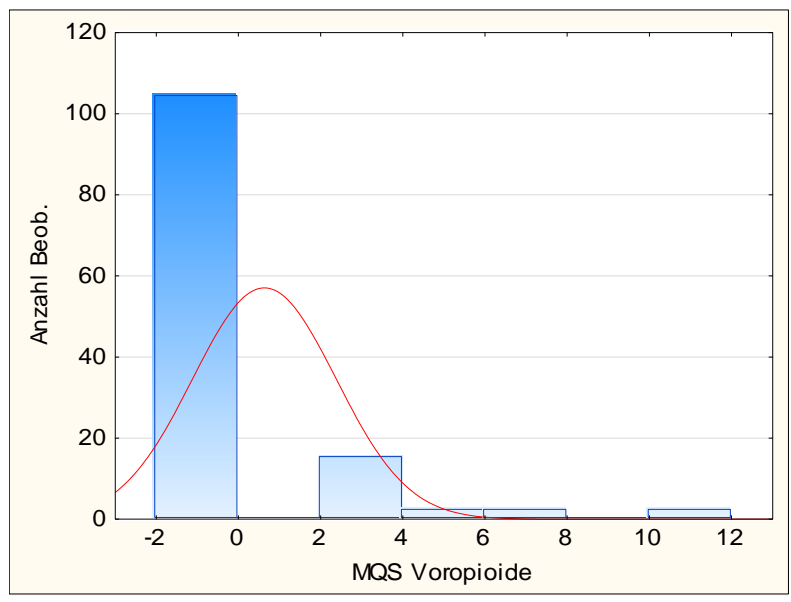

Abbildung 22: Histogramm MQS

"Vorbehandlung mit Opioiden“

Auch bei der MQS „Vorbehandlung mit Nichtopioiden“ (einschließlich Koanalgetika) handelt es sich bei den 125 vollständigen Patientendaten nicht um eine Normalverteilung, da der K.S.-Test einen p-Wert von <0,01 hat und in Abbildung $23 \mathrm{zu}$ sehen ist. 50 Probanden nahmen keine Nicht-Opioide vor der Operation ein. Die restlichen $60 \%$ nahmen nicht-opioidhaltige Schmerzmedikamente ein und das teilweise in hohen Dosen. Der Mittelwert beträgt 3,6 mit einer Standardabweichung von 4,1.

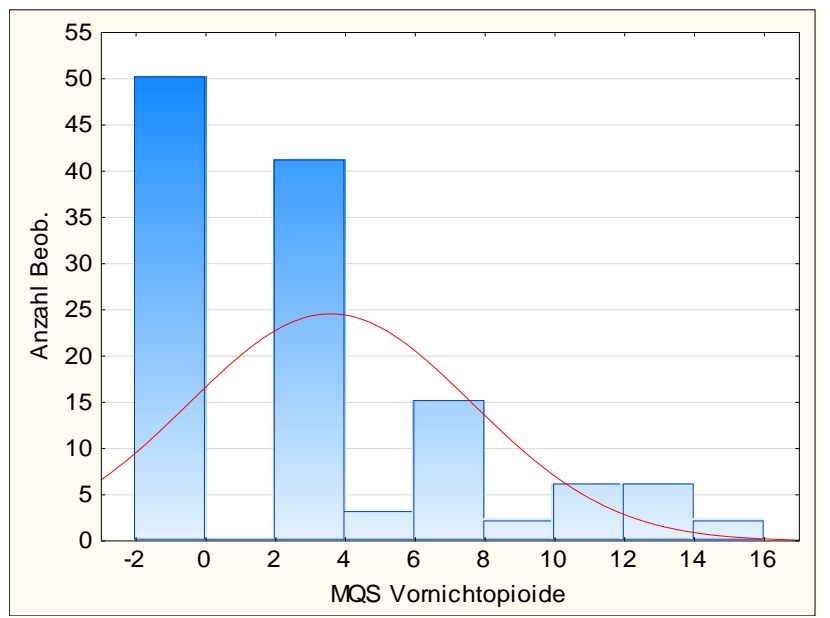

Abbildung 23: Histogramm MQS „Vorbehandlung mit Nichtopioiden" 


\subsubsection{NRS Schmerz in den letzten 3 Monaten}

Aufgrund diverser unterschiedlicher Schmerzparameter, welche initial erhoben wurden, verglichen wir die unterschiedlichen Variablen zunächst miteinander, um mit der geeignetsten Variable weiterarbeiten zu können.

Tabelle 2: Unterschiede der einzelnen Schmerzskalen in den letzten drei Monaten

\begin{tabular}{|l|l|l|l|l|}
\hline & Mittelwert & $\begin{array}{l}\text { Standard- } \\
\text { abweichung }\end{array}$ & Median & Range \\
\hline $\begin{array}{l}\text { Stärkster Schmerz in } \\
\text { den letzten 3 Monaten }\end{array}$ & 7,7 & 1,771 & 8 & 9 \\
\hline $\begin{array}{l}\text { Stärkster Hüftschmerz } \\
\text { in den letzten 3 } \\
\text { Monaten }\end{array}$ & 8,1 & 1,739 & 8 & 9 \\
\hline $\begin{array}{l}\text { Durchschnittlicher } \\
\text { Schmerz in den } \\
\text { letzten 3 Monaten }\end{array}$ & 5,74 & 1,849 & 6 & 9 \\
\hline $\begin{array}{l}\text { Durchschnittlicher } \\
\text { Hüftschmerz in den } \\
\text { letzten 3 Monaten }\end{array}$ & 5,75 & 1,817 & 5,5 & 9 \\
\hline
\end{tabular}

Durch die hohe Korrelation sowohl bei dem stärksten Schmerz (Abbildung 24) mit einem Mittelwert von 7,7 und Hüftschmerz mit einem Mittelwert von 8,1 in den letzten 3 Monaten, als auch bei dem durchschnittlichen Schmerz (Abbildung 25) mit einem Mittelwert von 5,7 und durchschnittlichen Hüftschmerz mit einem Mittelwert von 5,7 in den letzten 3 Monaten lässt sich vermuten, dass die Patienten die unterschiedlichen Schmerzen nicht gut voneinander trennen konnten, bzw. für die meisten Patienten der chronische Hüftschmerz führend war.

Die folgenden Darstellungen der Schmerzparameter in Scatterplots dienen lediglich der Veranschaulichung der Parameter. Statistisch sind sie so nicht verwertbar. 


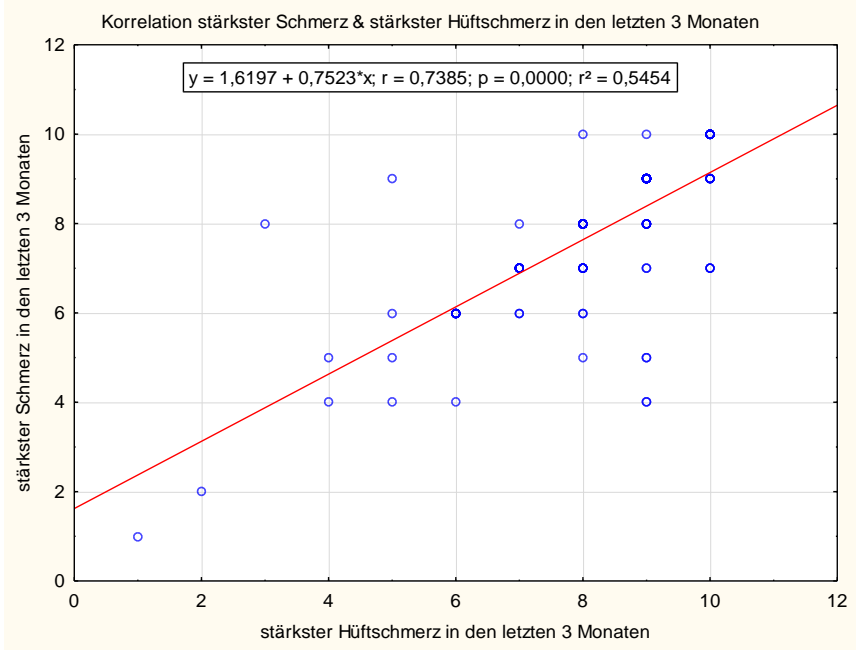

Abbildung 24: Scatterplot stärkster Schmerz vs. stärkster Hüftschmerz in den letzten drei Monaten

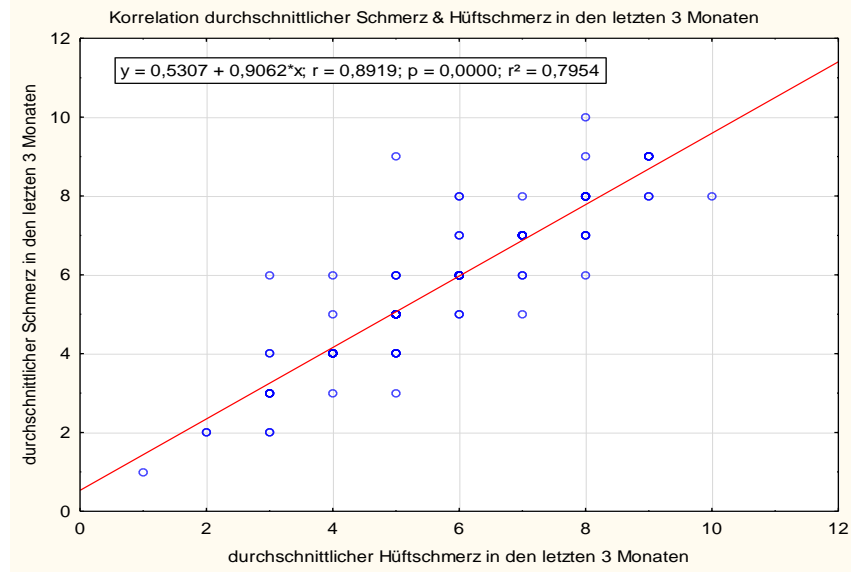

Abbildung 25: Scatterplot durchschnittlicher Schmerz vs. durchschnittlicher Hüftschmerz in den letzten drei Monaten

Da zudem im Chronic Pain Grade nach den Schmerzen der letzten drei Monate gefragt wird und spezifiziertere Fragen bezüglich der Schmerzen im WOMAC Schmerz Teil gestellt werden, haben wir eine unnötige Überschneidung der Fragen verhindert, indem wir die Variable stärkster Schmerz und die Variablen stärkster Hüftschmerz und durchschnittlicher Hüftschmerz in den letzten drei Monaten nicht in die multiplen Regressionen mit hineingenommen haben. Deshalb haben wir nur einen Messparameter, den durchschnittlichen Schmerz in den letzten drei Monaten als Variable in der weiteren statistischen Analyse verwendet. 
Diese Variable konnte bei 120 Patienten erhoben werden. Der Mittelwert beträgt 5,7 mit einer Standardabweichung von 1,8. Mit einem p-Wert <0,05 im K.S.-Test kann man keine Normalverteilung identifizieren, obwohl sie graphisch (Abbildung 26) nicht auszuschließen ist.

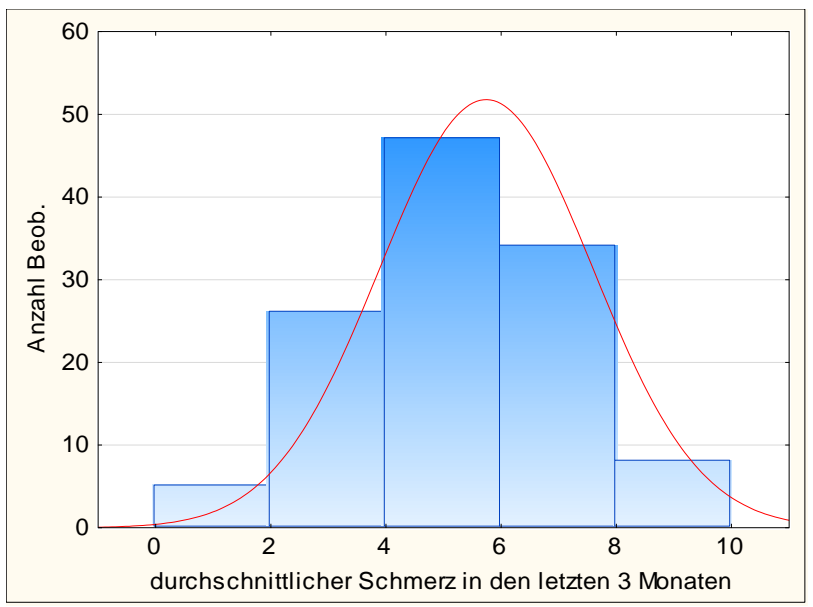

Abbildung 26: Histogramm durchschnittlicher Schmerz in den letzten drei Monaten

\subsection{Geschlechtsspezifische Unterschiede}

\subsubsection{Berechnung}

Insgesamt wurden 73 weibliche und 52 männliche Patienten in die Auswertung miteingeschlossen. Dabei sind die männlichen Patienten im Schnitt 6,7 Jahre älter. Der jüngste männliche Patient war 38 und der älteste 88 Jahre alt zum Zeitpunkt der Erhebung. Der Mittelwert beträgt 67,1 Jahre. Die jüngste weibliche Patientin hingegen war am Tag der Aufnahme 24 Jahre und die älteste 84 Jahre alt. Durch den Mittelwert errechnet waren sie im Schnitt 60,4 Lebensjahre alt. In Tabelle 3 werden die Ergebnisparameter nach Geschlecht getrennt dargestellt.

Alle Variablen, die im Kolmogorov-Smirnov Test nicht signifikant waren, gelten als normalverteilt. Diese Variablen wurden dann in einem weiteren Schritt mit dem T-Test auf Signifikanz bezüglich eines geschlechtsspezifischen Unterschiedes geprüft. 
Alle nicht-normalverteilten Variablen wurden in einem folgenden Schritt mithilfe des Mann-Whitney-U-Test auf Signifikanz geprüft.

Tabelle 3: Geschlechtsspezifische Unterschiede

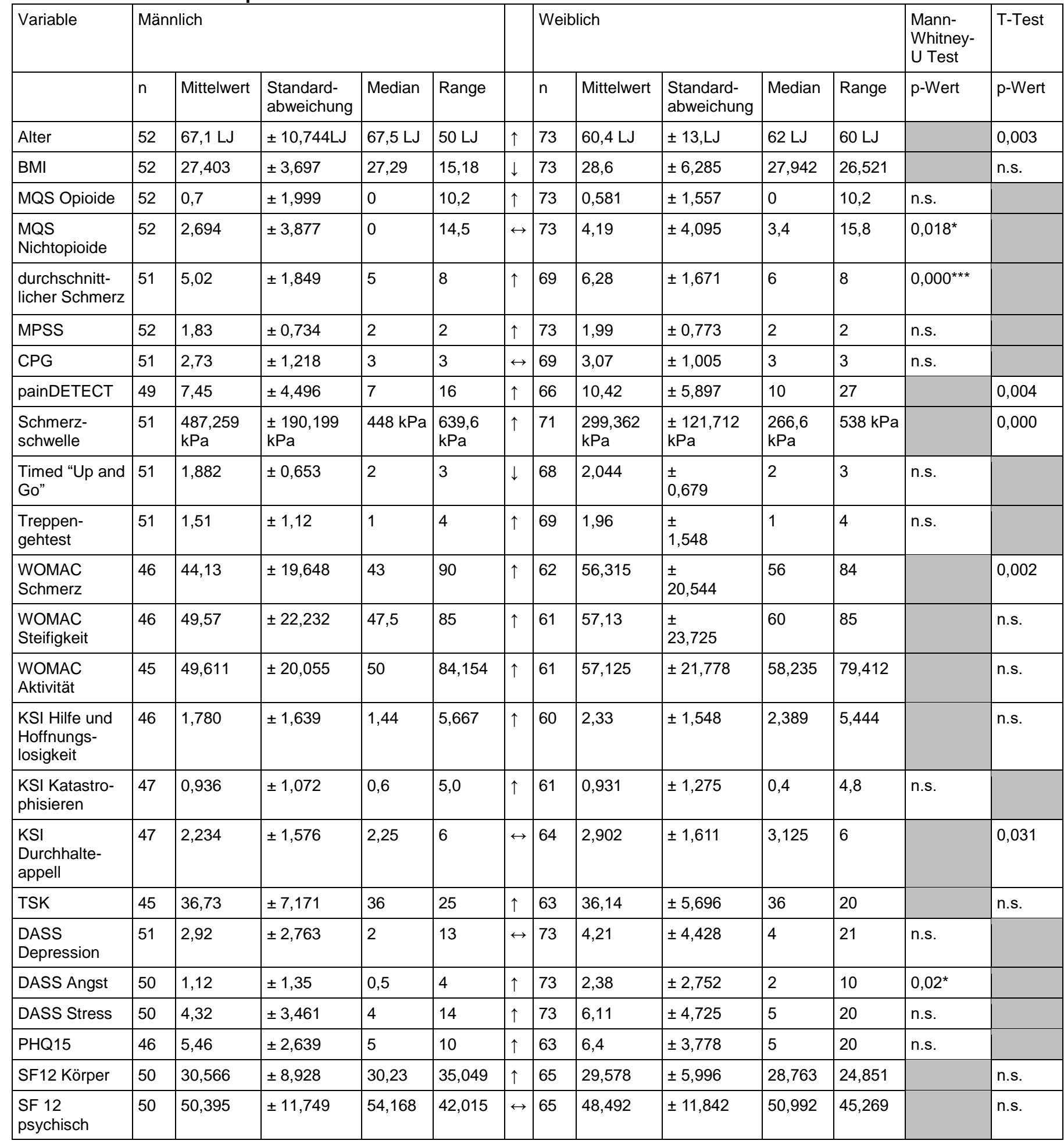




\subsubsection{Physische und klinische Parameter}

Frauen wurden mit einem Durchschnittsalter von 60,4 LJ signifikant früher operiert als Männer mit 67,1 LJ.

Beim Parameter durchschnittlicher Schmerz in den letzten drei Monaten erkennt man deutlich, dass die Frauen einen höheren Wert beim Schmerzscore angeben, als die Männer.

Auch beim painDETECT erreicht das weibliche Patientenkollektiv einen deutlich höheren Wert als das Männliche.

Bei dem Treppengehversuch gelangten die meisten Patienten in die erste Gruppe. Vom männlichen Patientenkollektiv waren es insgesamt 38 von 51 , also $74,5 \%$, und von den Frauen 44 von 69, also $64 \%$.

Bei der Schmerzschwelle (Abbildung 27) hat das männliche Patientenkollektiv ( $n=51)$ eine weit höhere Schmerzschwelle als die Frauen $(n=71)$. Bei den Männern erstreckt sich die Spannweite der Schmerzschwelle von 189,4 bis 829, wohingegen die weibliche Schmerzschwelle in diesem Patientenkollektiv bereits bei 98 anfängt und nur bis 636 geht.

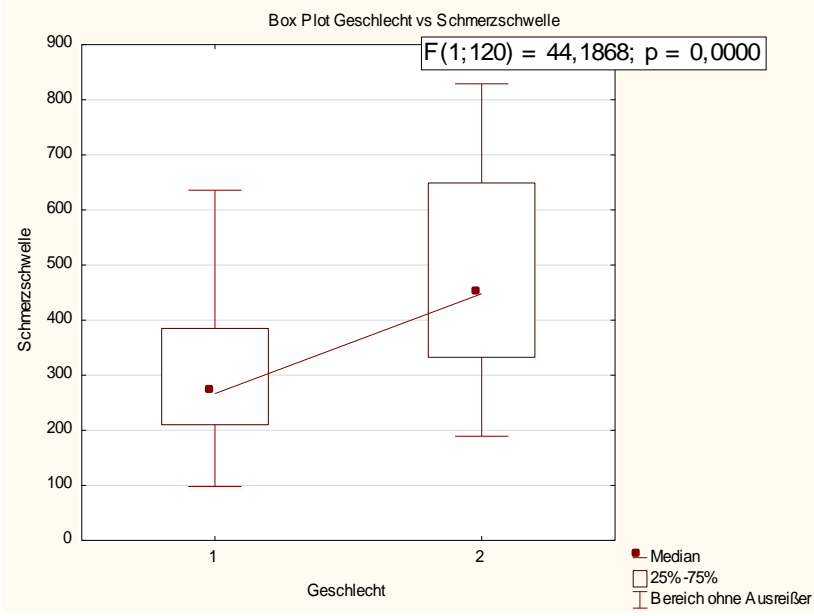

Abbildung 27: Boxplot Geschlechtervergleich Schmerzschwelle in kPA 1 = weiblich; 2 = männlich 
Auch beim WOMAC gibt es geschlechtsspezifische Unterschiede.

So ist bei dem WOMAC Schmerzscore (Abbildung 28) der Mittelwert des männlichen Patientenkollektivs $(n=52)$ niedriger, als der des weiblichen $(n=62)$, obwohl der Unterschied, was die Maximalwerte angeht, nur gering ist. So sind das Minimum der Männer 4 und das der Frauen 10. Das Maximum beträgt hingegen bei beiden Geschlechtern 94.

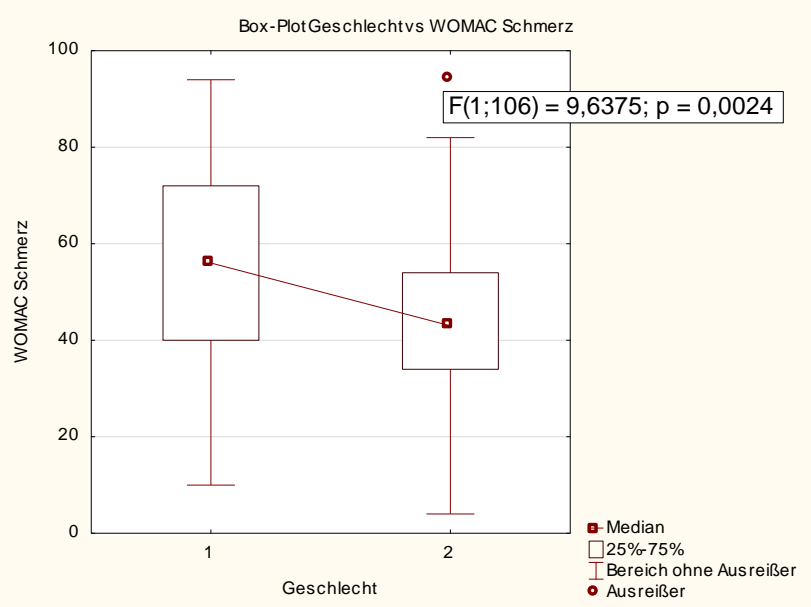

Abbildung 28: Boxplot Geschlechtervergleich WOMAC-Schmerz 1 = weiblich; 2 = männlich

Auch die anderen beiden WOMAC-Scores Steifigkeit und Aktivität weisen eine Differenz auf, wobei stets das männliche Kollektiv unter dem Mittelwert des weiblichen Kollektivs liegt.

Interessant ist, dass insgesamt 68,5 \% der Frauen $(n=73)$, aber nur 48,1 \% der Männer $\mathrm{n}=52$ ) präoperativ Mittel gegen Schmerzen nehmen. 


\subsubsection{Psychologische Parameter}

Das Interessante beim DASS Depression (Abbildung 29) sieht man an den Maximalwerten. Beim männlichen Patientenkollektiv $(n=50)$ ist das Maximum 13. Die Frauen ( $n=73$ ) hingegen haben zwar nur einen geringfügig höheren Mittelwert, aber das Maximum beträgt 21 .

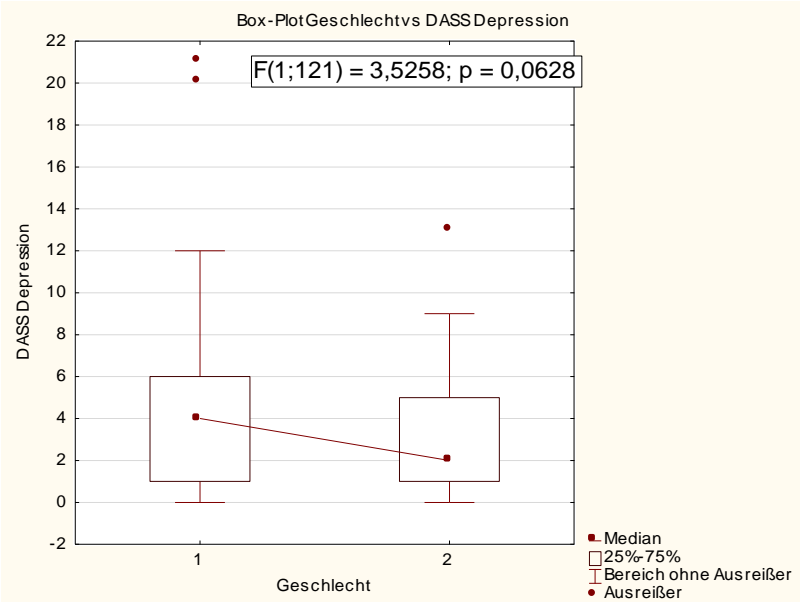

Abbildung 29: Boxplot Geschlechtervergleich beim DASS-Depression 1 = weiblich; 2 = männlich

Auch beim DASS-Angst (Abbildung 30) erkennt man deutliche Unterschiede bei den Maximalwerten. Die 73 Frauen haben einen Maximum von 10 deutlich über dem Maximum von 4 der 50 Männer. Dieser Unterschied ist jedoch nicht signifikant. 


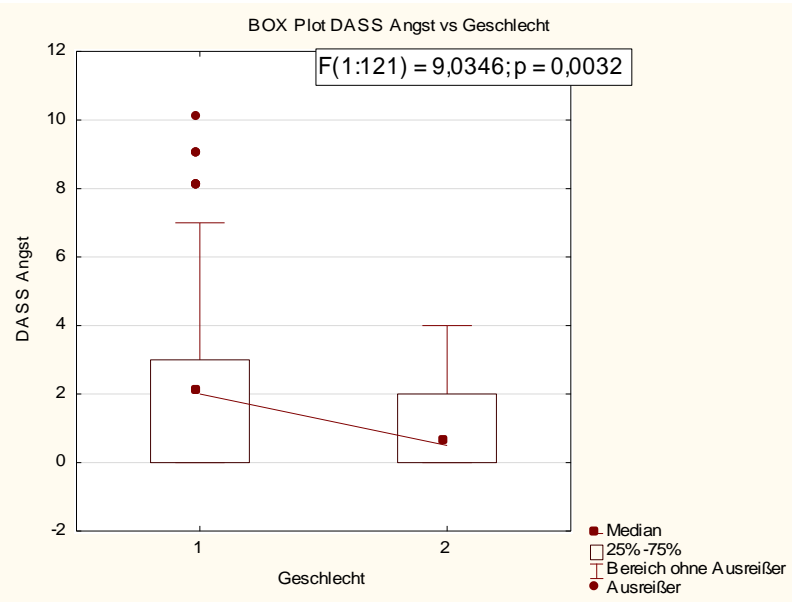

Abbildung 30: Boxplot Geschlechtervergleich beim DASS-Angst 1 = weiblich; 2 = männlich

Beim DASS-Stress (Abbildung 31) erreichen 50 \% der männlichen Patienten einen Wert von 0 (25 von 50 ), bei den weiblichen Patienten sind es hingegen nur 33 \% (24/73).

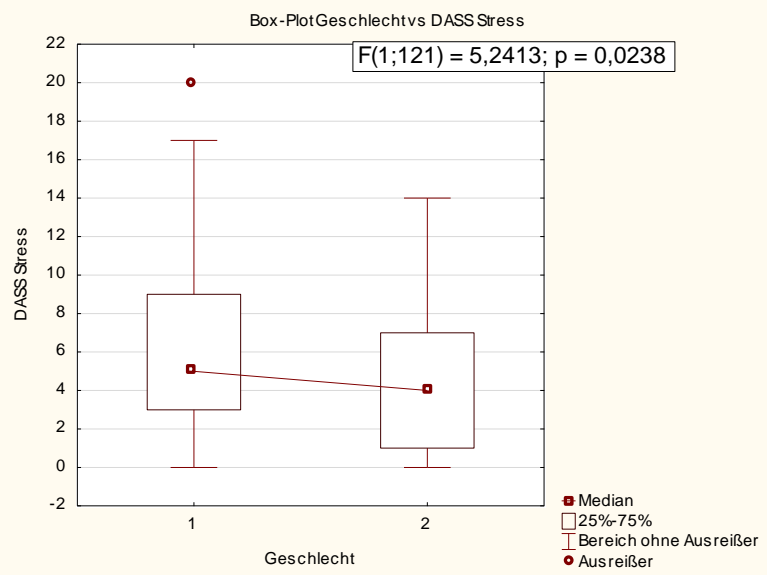

Abbildung 31: Boxplot Geschlechtervergleich beim DASS-Stress 1 = weiblich; 2 = männlich

Auch beim PHQ15 gab es geschlechtsspezifische Unterschiede in den Maxima, wo das weibliche Kollektiv einen Wert von 22 erreichte und das Männliche lediglich 12, jedoch war der Unterschied bei den Mittelwerten gering. 


\subsection{Auswahl der Endpunkte}

Aus den vorliegenden QUIPS-Daten zum postoperativen Schmerzverlauf der Tage 1, 3, 5 und 7 wurde eine Auswahl getroffen. Wir legten den ersten Tag nach der Operation fest, um die direkten Einflüsse der Operation festzustellen, und für den 5. Tag nach der Operation, da an diesem Tag die Probanden bereits größtenteils mobil waren. Den 3. Tag verwendeten wir nicht, da noch nicht alle Patienten mobilisiert wurden und am 7. Tag wurden bereits einige Patienten entlassen.

\subsubsection{Schmerzparameter}

Der geringste Schmerz war aus unserer Sicht als Endpunkt nicht geeignet, da in der Studeinpopulation große Unterschiede in der präoperativen Medikamenteneinnahme bestand.

Um die geeignetsten Parameter für die Endpunkte zu erfassen, haben wir die in Frage kommenden Variablen miteinander verglichen. So stellten wir fest, dass es zwischen dem Belastungsschmerz und dem stärksten Schmerz sowohl am 1. pOP Tag (Abbildung 32) als auch am 5. pOP Tag (Abbildung 33) nur einen geringfügigen Unterschied gab. Unter den Patienten gab es aber weit gefächert verschiedene Arten von Belastungen, da manche noch sportlich aktiv waren und andere nur noch teilweise häusliche Aufgaben verrichten konnten. Daher legten wir den maximalen Schmerz sowohl am ersten postoperativen Tag als auch am 5. postoperativen Tag als Endpunkt fest.

Wie bereits zuvor erwähnt, dienen die Scatterplots bei den Schmerzparametern lediglich zur Veranschaulichung und nicht zur statistischen Auswertung. 


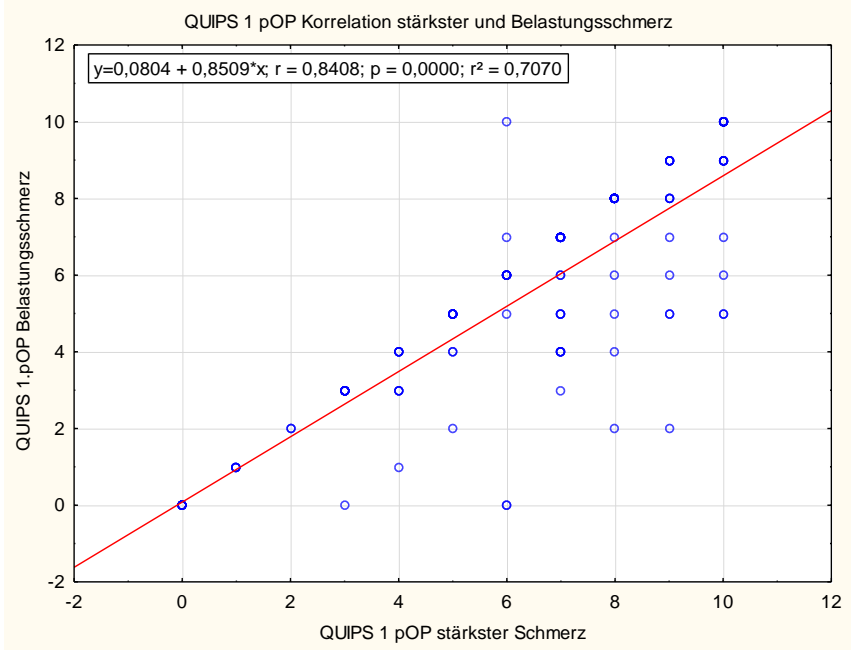

Abbildung 32: Scatterplot stärkster Schmerz vs. Belastungsschmerz am 1. pOP Tag

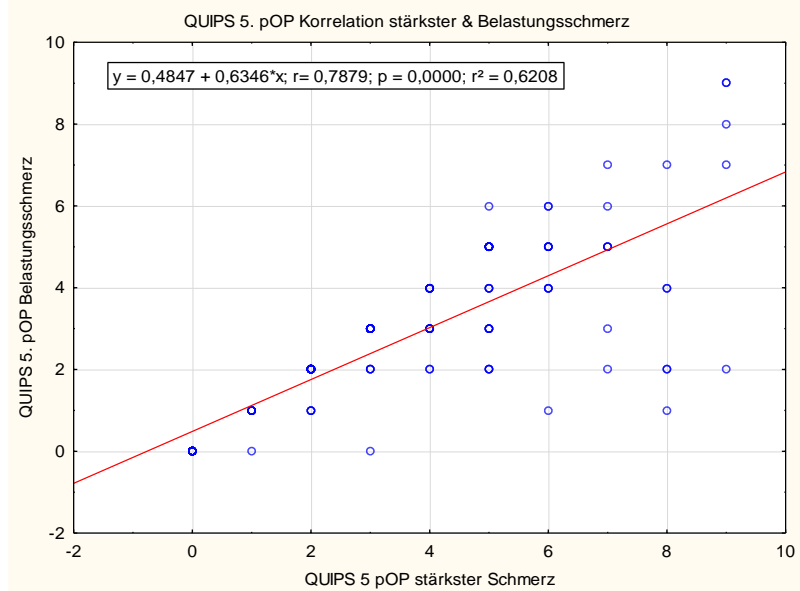

Abbildung 33: Scatterplot stärkster Schmerz vs. Belastungsschmerz am 5. pOP Tag

\subsubsection{Morphinverbrauch}

Anhand der Histogramme (Abbildungen 34 und 35) kann man erkennen, dass die Patienten im Verlauf (1. versus 5. Tag) weniger starke Maximalschmerzen angaben. Am ersten pOP Tag gibt es Daten von allen 125 eingeschlossenen Patienten. Der p-Wert im K.S.-Test beträgt $>0,01$, sodass der Parameter als nicht normalverteilt gilt. Der Mittelwert beträgt 6,3 mit einer Standardabweichung von 2,9. Insgesamt gaben $n=8$ Patienten keine Schmerzen an. Am 5. pOP Tag sind es nur noch 123 Patientendaten mit einem p- 
Wert von $>0,05 \mathrm{im} \mathrm{K.S.-Test.} \mathrm{Der} \mathrm{Mittelwert} \mathrm{ist} \mathrm{3,7} \mathrm{mit} \mathrm{einer} \mathrm{Standardabweichung} \mathrm{von}$ 2,5. Insgesamt geben am 5. pOP $n=16$ Patienten keine Schmerzen mehr an.

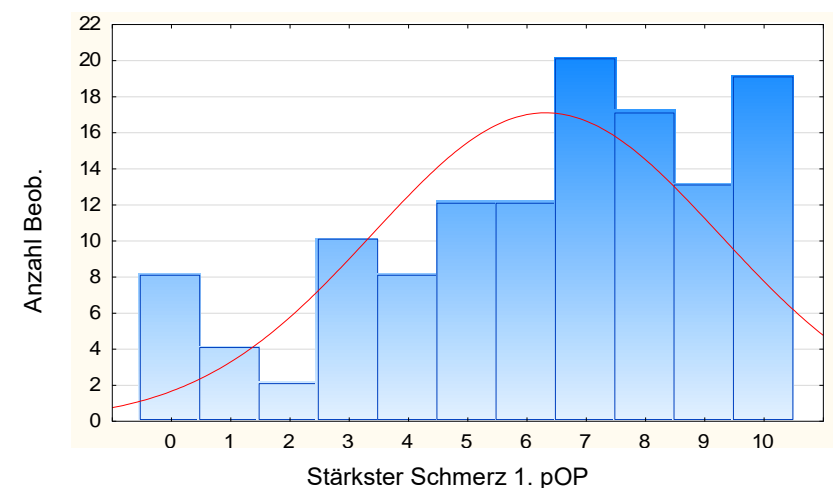

Abbildung 34: Histogramm stärkster Schmerz 1. pOP Tag

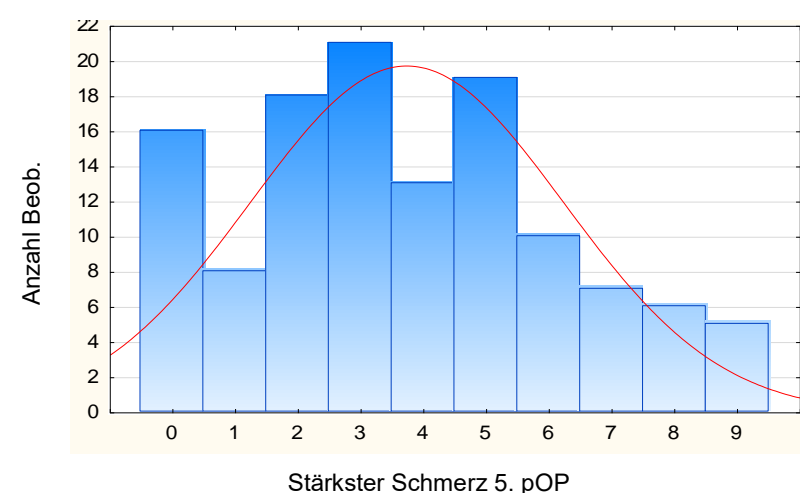

Abbildung 35: Histogramm stärkster Schmerz 5. pOP Tag

Für das zugehörige Morphinäquivalent entschieden wir uns am ersten postoperativen Tag für einen Kumulativwert von 48 Stunden, gerechnet vom Tag der Operation bis zum Ende des ersten postoperativen Tages. Der morphinäquivalente Gebrauch während der Operation und im Aufwachraum bzw. auf der Intensivstation wurde miteingerechnet. Für diese Berechnung gibt es 122 Patientendatensätze, der Mittelwert beträgt 120,6mg mit einer Standardabweichung von 49,4. Äquivalent zum maximalen Schmerz wurde als zweiter Messpunkt das Morphinäquivalent vom 5. pOP Tag genommen. Bei diesen Endpunkten sieht man am 1. pOP Tag noch eine Normalverteilung im Histogramm (Abbildung 36) und anhand eines p-Wertes von $>0,15 \mathrm{im}$ K.S.-Test. Diese 
Normalverteilung verschwindet statistisch gesehen während des stationären Aufenthaltes sodass man am 5. pOP in Abbildung 37 keine Normalverteilung im Histogramm erkennen kann. Hier beträgt der p-Wert im K.S.-Test $<0,01$. Am 5. pOP Tag gibt es Werte von 123 Patienten, der Mittelwert ist 25,9 mit einer Standardabweichung von 23,3. Auch für die Opioidgabe wird zum 5. Tag hin eine deutlich geringere Einnahme deutlich.

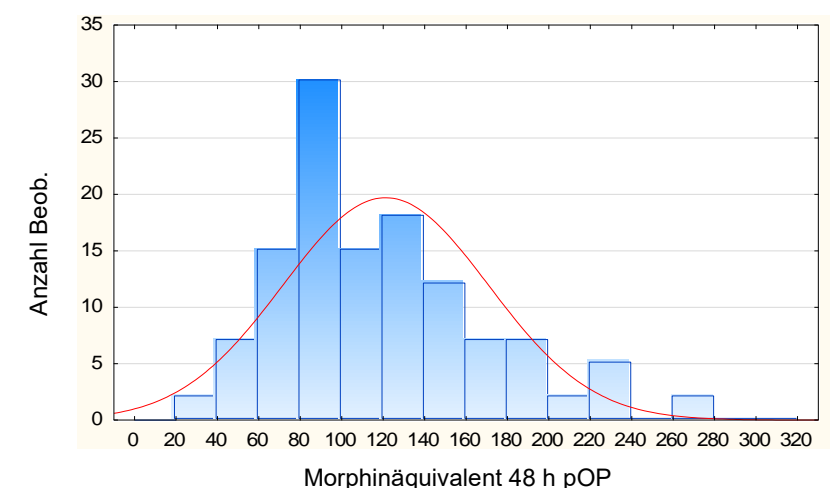

Abbildung 36: Histogramm Morphinäquivalent 48 h pOP

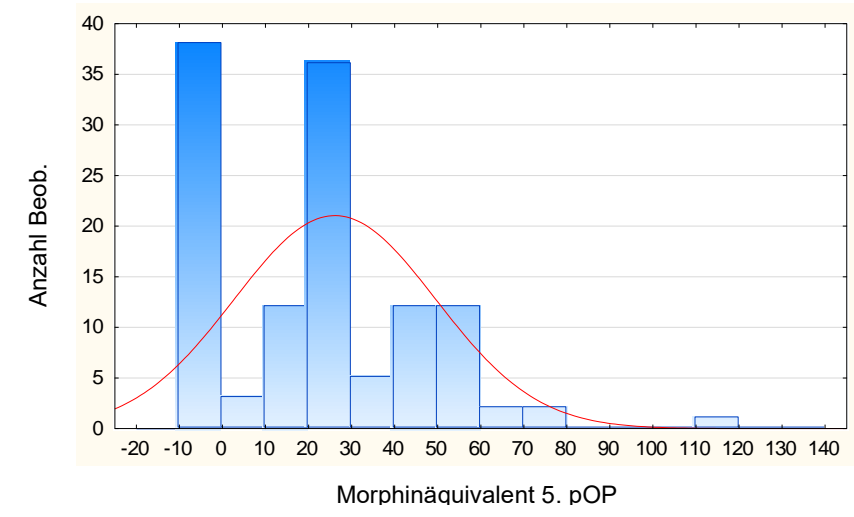

Abbildung 37: Histogramm Morphinäquivalent 5. pOP Tag

Wie man anhand Abbildung 38 erkennen kann, korrelieren die stärksten Schmerzen am ersten postoperativen Tag positiv mit dem Morphinäquivalent bis einschließlich des ersten Tages mit $r=0,303$. Dennoch zeigt sich eine breite Streuung bei dem Morphinäquivalent. Es gibt zudem viele Probanden mit der Angabe von hohen Schmerzspitzen, welche dennoch eher wenig Morphinäquivalent erhalten haben. 


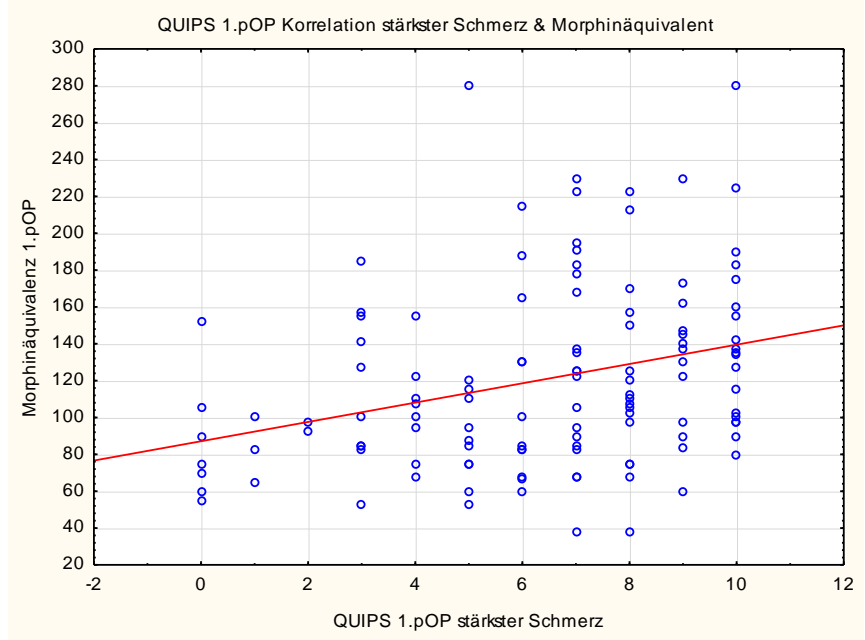

Abbildung 38: Korrelation stärkster Schmerz \& Morphinäquivalent 1.pOP

Betrachtet man im Vergleich zur Abbildung 38 nun Abbildung 39, so zeigt sich auch hier eine positive Korrelation am 5. postoperativen Tag vom stärksten Schmerz zum Morphinäquivalent mit $r=0,325$. Insgesamt sind die Werte nicht so breit gestreut, wie am ersten postoperativen Tag und es gibt nur einen Ausreißer. Auch wurde im Vergleich zum ersten postoperativen Tag am 5. postoperativen Tag eine geringere Intensität der Schmerzspitzen angegeben und insgesamt ein deutlich geringeres Morphinäquivalent verschrieben, dennoch sieht man anhand Abbildung 39, dass Probanden mit stärksten Schmerzen auf der NRS $>5$ teilweise kein Morphin erhielten, bzw. anforderten.

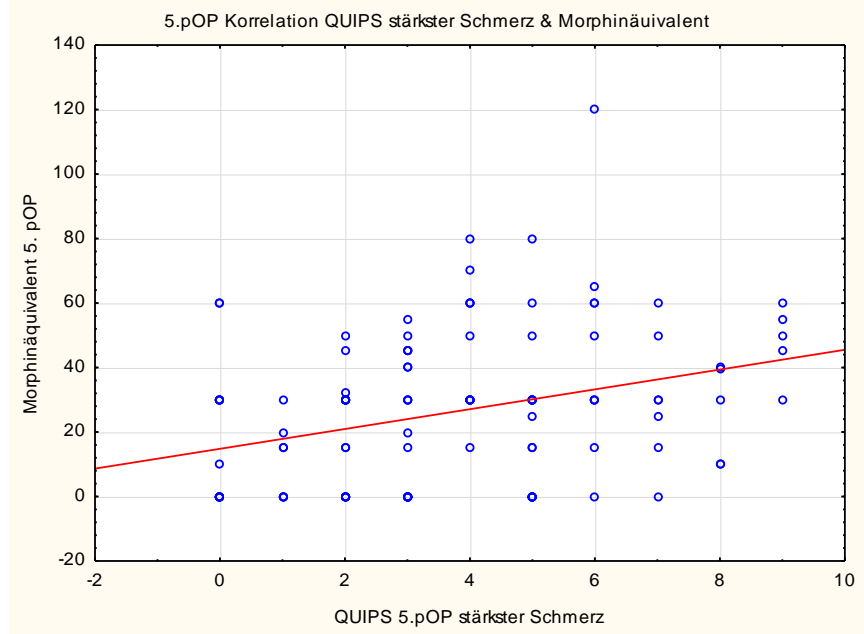

Abbildung 39: Korrelation stärkster Schmerz \& Morphinäquivalent 5. pOP 
Natürlich korrelieren die ausgewählten Endpunkte etwas miteinander, da Patienten mit mehr Schmerz möglicherweise auch mehr Opioide brauchen, jedoch ist der Zusammenhang mit einem $r$ von $<0,35$ eher schwach und weist auf die Relevanz anderer Faktoren hin als die Schmerzientensität, um die erhaltene Menge an Opioiden zu erklären.

\subsection{Korrelationstabelle}

Die Korrelationstabellen dienen der Übersicht, inwiefern die einzelnen untersuchten Parameter selbst im Zusammenhang untereinander stehen. Grau unterlegt sind alle Werte, welche eine Korrelation mit einem $r>0,25$ aufweisen. Werte größer oder gleich 0,5 sind zusätzlich einer Nuance dunkler unterlegt.

Deutlich zu erkennen ist, dass gerade die Subgruppen der Fragebögen DASS und WOMAC untereinander mit über 0,5 korrelieren. Auch die Subgruppen des KSI korrelieren teilweise mit über 0,5 mit den Fragebögen WOMAC und DASS.

Die schmerzspezifischen Fragebögen wie painDETECT, MPSS und CPG weisen eine starke Korrelation zueinander auf. Insbesondere der CPG mit dem painDETECT mit einem Wert über 0,5 .

Auch die Funktionstest Timed „Up and Go“- sowie Treppengehtest stehen in einem starken Zusammenhang zueinander.

Der WOMAC-Schmerz korreliert sowohl stark mit dem durchschnittlichen Schmerz der letzten 3 Monate, als auch mit >0,6 mit dem CPG.

Insgesamt zeigt sich, dass wir zwar viele Parameter benutzt haben, welche eine starke Korrelation zueinander aufweisen, jedoch auch ein sehr breites Spektrum erfasst haben, welches nur geringfügig miteinander korreliert. 
Tabelle 4: Korrelationstabelle Teil 1

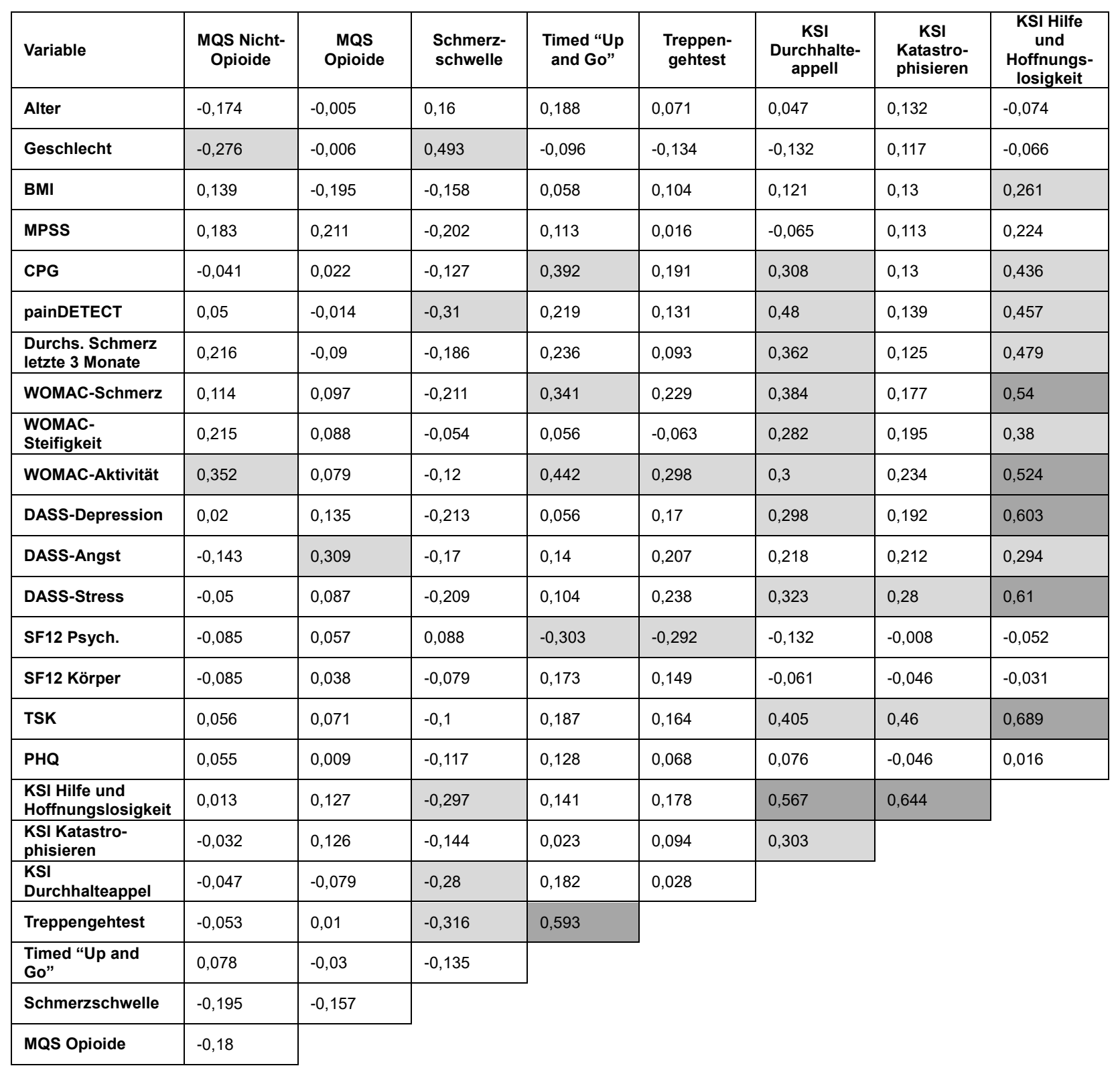


Tabelle 5: Korrelationstabelle Teil 2

\begin{tabular}{|c|c|c|c|c|c|c|c|c|}
\hline Variable & PHQ & TSK & $\begin{array}{c}\text { SF12 } \\
\text { Körper }\end{array}$ & $\begin{array}{l}\text { SF12 } \\
\text { Psych. }\end{array}$ & $\begin{array}{l}\text { DASS- } \\
\text { Stress }\end{array}$ & $\begin{array}{l}\text { DASS- } \\
\text { Angst }\end{array}$ & $\begin{array}{c}\text { DASS- } \\
\text { Depression }\end{array}$ & $\begin{array}{l}\text { WOMAC- } \\
\text { Aktivität }\end{array}$ \\
\hline Alter & 0,042 & $-0,12$ & 0,165 & $-0,134$ & $-0,165$ & 0,213 & $-0,186$ & $-0,0006$ \\
\hline Geschlecht & $-0,151$ & 0,076 & 0,101 & 0,144 & $-0,229$ & $-0,225$ & $-0,192$ & $-0,164$ \\
\hline BMI & $-0,038$ & 0,2 & $-0,111$ & 0,104 & 0,157 & $-0,076$ & 0,172 & 0,369 \\
\hline MPSS & 0,059 & 0,08 & 0,021 & 0,124 & 0,193 & 0,094 & 0,172 & 0,344 \\
\hline CPG & 0,105 & 0,246 & 0,152 & $-0,101$ & 0,348 & 0,105 & 0,376 & 0,648 \\
\hline painDETECT & 0,039 & 0,303 & 0,118 & $-0,138$ & 0,33 & 0,378 & 0,457 & 0,422 \\
\hline $\begin{array}{l}\text { Durchs. Schmerz } \\
\text { letzte } 3 \text { Monate }\end{array}$ & $-0,083$ & 0,327 & 0,037 & $-0,024$ & 0,311 & 0,194 & 0,316 & 0,634 \\
\hline $\begin{array}{l}\text { WOMAC- } \\
\text { Schmerz }\end{array}$ & 0,034 & 0,325 & 0,074 & $-0,038$ & 0,446 & 0,187 & 0,38 & 0,859 \\
\hline $\begin{array}{l}\text { WOMAC- } \\
\text { Steifigkeit }\end{array}$ & 0,03 & 0,313 & 0,071 & 0,035 & 0,32 & 0,194 & 0,26 & 0,586 \\
\hline WOMAC-Aktivität & $-0,072$ & 0,394 & $-0,011$ & 0,006 & 0,426 & 0,247 & 0,36 & \\
\hline $\begin{array}{l}\text { DASS- } \\
\text { Depression }\end{array}$ & 0,104 & 0,433 & 0,101 & $-0,083$ & 0,788 & 0,504 & & \\
\hline DASS-Angst & 0,008 & 0,127 & 0,277 & $-0,227$ & 0,497 & & & \\
\hline DASS-Stress & 0,012 & 0,427 & 0,033 & $-0,08$ & & & & \\
\hline SF12 Psych. & $-0,313$ & 0,038 & $-0,089$ & & & & & \\
\hline SF12 Körper & $-0,131$ & 0,047 & & & & & & \\
\hline TSK & $-0,17$ & & & & & & & \\
\hline
\end{tabular}

Tabelle 6: Korrelationstabelle Teil 3

\begin{tabular}{|c|c|c|c|c|c|c|c|c|}
\hline Variable & $\begin{array}{l}\text { WOMAC- } \\
\text { Steifigkeit }\end{array}$ & $\begin{array}{l}\text { WOMAC- } \\
\text { Schmerz }\end{array}$ & $\begin{array}{l}\text { Durchs. } \\
\text { Schmerz } \\
\text { letzte } 3 \\
\text { Monate }\end{array}$ & painDETECT & CPG & MPSS & BMI & Geschlecht \\
\hline Alter & $-0,088$ & $-0,182$ & $-0,18$ & $-0,296$ & $-0,074$ & $-0,227$ & $-0,059$ & 0,276 \\
\hline Geschlecht & $-0,154$ & $-0,293$ & $-0,345$ & $-0,281$ & $-0,091$ & $-0,054$ & $-0,101$ & \\
\hline BMI & 0,202 & 0,247 & 0,318 & 0,22 & 0,264 & 0,246 & & \\
\hline MPSS & 0,205 & 0,333 & 0,164 & 0,252 & 0,292 & & & \\
\hline CPG & 0,338 & 0,651 & 0,518 & 0,511 & & & & \\
\hline painDETECT & 0,299 & 0,463 & 0,48 & & & & & \\
\hline $\begin{array}{l}\text { Durchs. } \\
\text { Schmerz letzte } \\
3 \text { Monate }\end{array}$ & 0,39 & 0,687 & & & & & & \\
\hline $\begin{array}{l}\text { WOMAC- } \\
\text { Schmerz }\end{array}$ & 0,604 & & & & & & & \\
\hline
\end{tabular}




\subsection{Univariate Regression}

Um aus der Vielfalt der Daten die wichtigsten Punkte herauszufinden, betrachteten wir unsere 25 Variablen, bestehend aus Alter, BMI, Geschlecht, CPG, MPSS, painDETECT, PHQ15, TSK, KSI Hilfe und Hoffnungslosigkeit, KSIKatastrophisieren und KSI Durchhalteappell, SF12 Körper und SF12 Psychisch, DASS-Depression, DASS-Angst und DASS-Stress, WOMAC-Schmerz, WOMAC-Steifigkeit und WOMAC-Alltagsaktivität, MQS Opioide, MQS Nichtopioide, durchschnittlicher Schmerz in den letzten 3 Monaten, Schmerzschwelle, Timed „Up and Go“-Score und „Treppengehtest“-Score, genauer. Wir prüften univariat deren Signifikanz im Hinblick auf die Endpunkte maximaler Schmerz (ordinal logit-Regression ohne modifiziertes $\mathrm{R}^{2}$ ) und Opioidverbrauch (lineare Regression mit korreliertem $\mathrm{R}^{2}$ ) je am 1. und 5. postoperativen Tag. Signifikant war eine Variable, wenn der $p$-Wert $<0,05$ war. Grau unterlegt sind die entsprechenden $R^{2}$ über 0,05 für die lineare Regression (Tabelle 7 ).

Dabei ist hervorzuheben, dass der CPG und der DASS-Depression mit allen Parametern in einem signifikanten Zusammenhang stehen, der DASS-Angst hingegen nur mit dem Schmerzempfinden.

Auch die Mobilität gemessen am Timed „Up and Go“-Test ist signifikant mit dem starken Schmerz verbunden und nur in den ersten $48 \mathrm{~h}$ mit dem Opioidverbrauch.

Der KSI Hilfe und Hoffnungslosigkeit steht mit drei Endpunkten in einem signifikanten Zusammenhang zueinander. Innerhalb der ersten $48 \mathrm{~h}$ jedoch nicht zum Opioidverbrauch.

Der MPSS hingegen ist nur signifikant für den fünfter postoperativen Tag, aber dort sowohl für den Opioidverbrauch als auch für das Schmerzempfinden. 
Tabelle 7: Univariate Regression: Eingangsparameter mit den gewählten Endpunkten

\begin{tabular}{|c|c|c|c|c|c|c|}
\hline \multirow[t]{2}{*}{ Variable } & \multirow{2}{*}{$\begin{array}{l}\text { Stärkster } \\
\text { Schmerz 1. pOP } \\
\text { p-Wert }\end{array}$} & \multirow{2}{*}{$\begin{array}{l}\begin{array}{l}\text { Stärkster } \\
\text { Schmerz } 5 . \\
\text { pOP }\end{array} \\
\text { p-Wert }\end{array}$} & \multicolumn{2}{|c|}{$\begin{array}{l}\text { Morphinäquivalent } 48 \\
\text { Stunden }\end{array}$} & \multicolumn{2}{|c|}{$\begin{array}{l}\text { Morphinäquivalent } 5 . \\
\text { pOP }\end{array}$} \\
\hline & & & $p$-Wert & korr. $\mathrm{R}^{2}$ & $\mathrm{p}$-Wert & korr. $\mathrm{R}^{2}$ \\
\hline Alter & n.s. & n.s. & 0,001 & 0,078 & n.s. & $-0,003$ \\
\hline Geschlecht & n.s. & n.s. & n.s. & 0,019 & n.s & $-0,004$ \\
\hline BMI & 0,012 & 0,021 & n.s. & 0,01 & n.s. & 0,006 \\
\hline CPG & 0,008 & 0,041 & 0,02 & 0,038 & 0,048 & 0,025 \\
\hline MPSS & n.s. & 0,002 & n.s. & 0,011 & 0,002 & 0,072 \\
\hline painDETECT & n.s. & 0,000001 & n.s. & $-0,002$ & n.s. & 0,024 \\
\hline PHQ15 & n.s. & n.s. & n.s. & $-0,001$ & n.s. & $-0,004$ \\
\hline TSK & n.s. & n.s. & n.s. & $-0,004$ & n.s. & $-0,009$ \\
\hline DASS-Depression & 0,001 & 0,003 & 0,005 & 0,057 & 0,01 & 0,047 \\
\hline DASS-Angst & 0,035 & 0,03 & n.s. & $-0,007$ & n.s. & $-0,008$ \\
\hline DAS- Stress & 0,002 & 0,005 & 0,033 & 0,03 & n.s. & 0,013 \\
\hline $\begin{array}{l}\text { KSI Hilfe und } \\
\text { Hoffnungs- } \\
\text { losigkeit }\end{array}$ & 0,003 & 0,0008 & n.s. & 0,005 & 0,037 & 0,033 \\
\hline $\begin{array}{l}\text { KSI } \\
\text { Katastrophisieren }\end{array}$ & n.s. & n.s. & n.s. & $-0,007$ & n.s. & $-0,009$ \\
\hline $\begin{array}{l}\text { KSI } \\
\text { Durchhalteappel }\end{array}$ & n.s. & n.s. & n.s. & 0,001 & n.s. & 0,018 \\
\hline SF12 Körper & n.s. & n.s. & n.s. & $-0,009$ & n.s. & $-0,009$ \\
\hline SF12 Psych. & n.s. & n.s. & n.s. & 0,012 & n.s. & $-0,003$ \\
\hline WOMAC-Schmerz & n.s. & n.s. & n.s. & 0,014 & 0,007 & 0,059 \\
\hline $\begin{array}{l}\text { WOMAC- } \\
\text { Steifigkeit }\end{array}$ & 0,043 & n.s. & n.s. & $-0,002$ & 0,03 & 0,03 \\
\hline WOMAC-Aktivität & 0,045 & n.s. & n.s. & $-0,002$ & n.s. & 0,014 \\
\hline $\begin{array}{l}\text { Durchs. Schmerz } \\
3 \text { Mon }\end{array}$ & 0,023 & 0,006 & n.s. & $-0,002$ & n.s. & 0,024 \\
\hline $\begin{array}{l}\text { Timed “Up and } \\
\text { Go"-Test }\end{array}$ & 0,027 & 0,043 & 0,037 & 0,029 & n.s. & $-0,009$ \\
\hline Treppengehtest & n.s. & 0,025 & n.s. & $-0,004$ & n.s. & $-0,009$ \\
\hline Schmerzschwelle & n.s. & 0,001 & 0,018 & 0,038 & n.s. & 0,006 \\
\hline MQS Opioide & n.s. & n.s. & n.s. & 0,005 & 0,00001 & 0,138 \\
\hline MQS Nichtopioide & n.s. & 0,039 & n.s. & 0,065 & n.s. & 0,004 \\
\hline
\end{tabular}




\subsubsection{Maximaler Schmerz 1. postoperativer Tag}

Beim maximalen Schmerz am 1. postoperativen Tag gab es insgesamt 10 signifikante Variablen.

Der DASS-Depression ist mit einem $p$-Wert von 0,001 univariant unter diesem Gesichtspunkt die signifikanteste Variable, dicht gefolgt von zwei weiteren psychologischen Parametern, dem DASS-Stress mit einem p-Wert von 0,002 und anschließend dem KSI Hilfe und Hoffnungslosigkeit mit einem p-Wert von 0,003. Nur noch der CPG fällt mit einem p-Wert unter 0,01 auf.

Das Alter hat in der univariaten Regression keine Signifikanz. Allerdings sieht man im Scatterplot (Abbildung 40) eine leichte Tendenz, welche darauf hinweist, dass ältere Patienten weniger Schmerzen angeben.

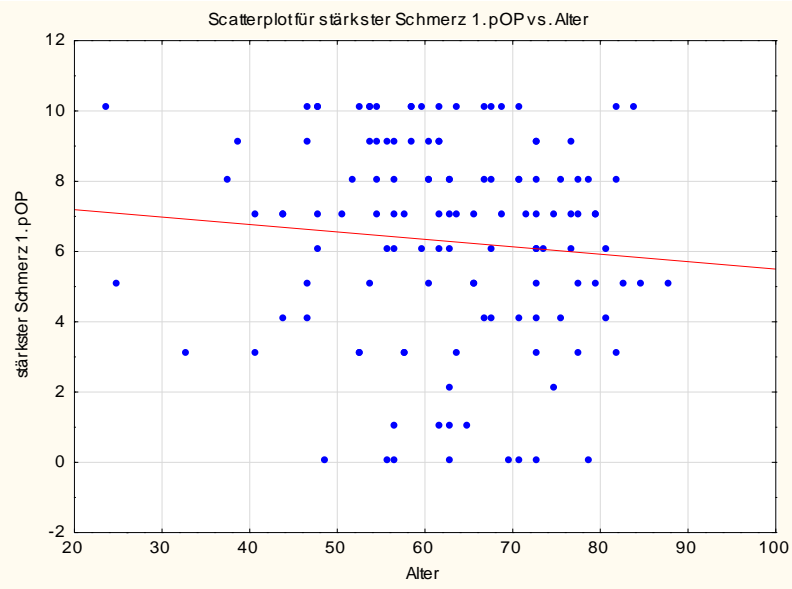

Abbildung 40: Scatterplot stärkster Schmerz 1. pOP vs. Alter 


\subsubsection{Maximaler Schmerz 5. postoperativer Tag}

Mit insgesamt 13 signifikanten Variablen fällt besonders der painDETECT mit einem $p$ Wert von 0,000001 auf und ist damit auch der signifikanteste Parameter in dieser Rechnung. Aber auch die psychologischen Variablen KSI Hilfe und Hoffnungslosigkeit, der DASS-Depression und der DASS-Stress fallen mit hohen signifikanten Werten auf. Des Weiteren haben noch der durchschnittliche Schmerz in den letzten 3 Monaten, die Schmerzschwelle und der MPSS p-Werte unter 0,01.

Am 5. pOP Tag hat die präoperative neuropathische Schmerzkomponente die stärkste Aussage bezüglich der postoperativen Schmerzen. Die Summenwerte des painDETECTs zeigen eine breite Verteilung und haben univariat bereits eine sehr hohe Signifikanz. Auch im Scatterplot (Abbildung 41) kann man den Zusammenhang dieses Parameters zum postoperativen Schmerz deutlich sehen.

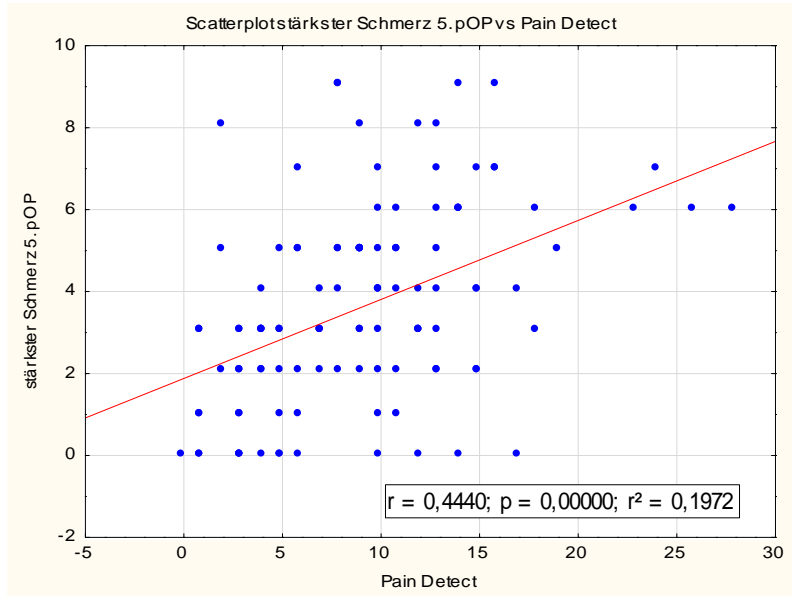

Abbildung 41: Scatterplot stärkster Schmerz 5. pOP vs painDETECT 
Auch am 5. pOP Tag lässt sich keine Signifikanz für die Variable Alter erkennen (Abbildung 42). Interessant ist allerdings der Vergleich zum 1. pOP Tag (Abbildung 40), wo die älteren Patienten eher weniger Schmerzen angaben, als die jüngeren. Am 5. pOP ist diese Tendenz umgekehrt. Ältere Probanden geben etwas stärkere Schmerzen an als jüngere, was aber keine Signifikanz hat.

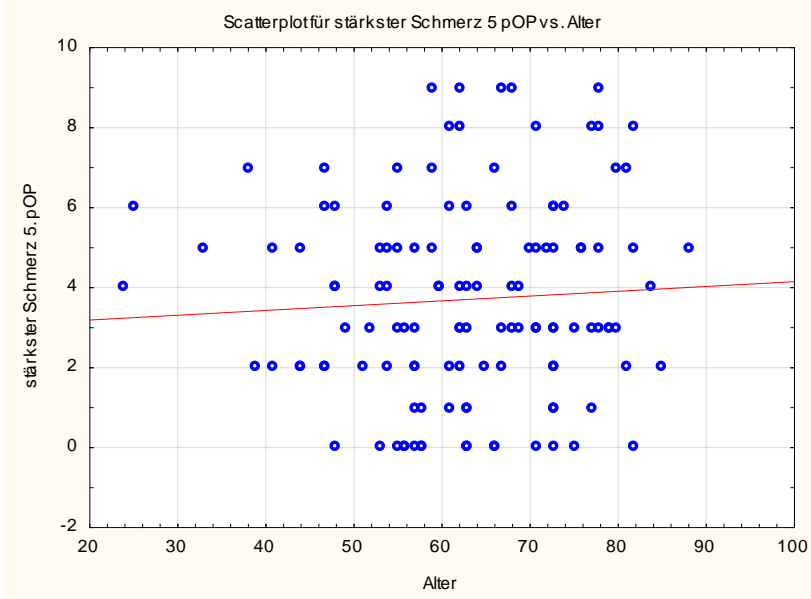

Abbildung 42: Scatterplot stärkster Schmerz 5. pOP vs. Alter

\subsubsection{Morphinverbrauch kumulativ 48 Stunden ab dem Operationstag}

Betrachtet man hingegen den Analgetikaverbrauch nach 48 Stunden so waren 6 präoperative Variablen signifikant. Es hatte bei diesem Endpunkt das Alter die höchste Signifikanz mit einem $p$-Wert von 0,001 dicht gefolgt vom DASS-Depression ( $p$-Wert $0,005)$.

Wie man gut in diesem Scatterplot erkennen kann, erhalten die Patienten mit höherem Alter weniger morphinäquivalente Schmerzmittel. Dieser Parameter geht aber nicht mit erhöhtem Schmerz einher (siehe Abbildungen 40 und 43). 


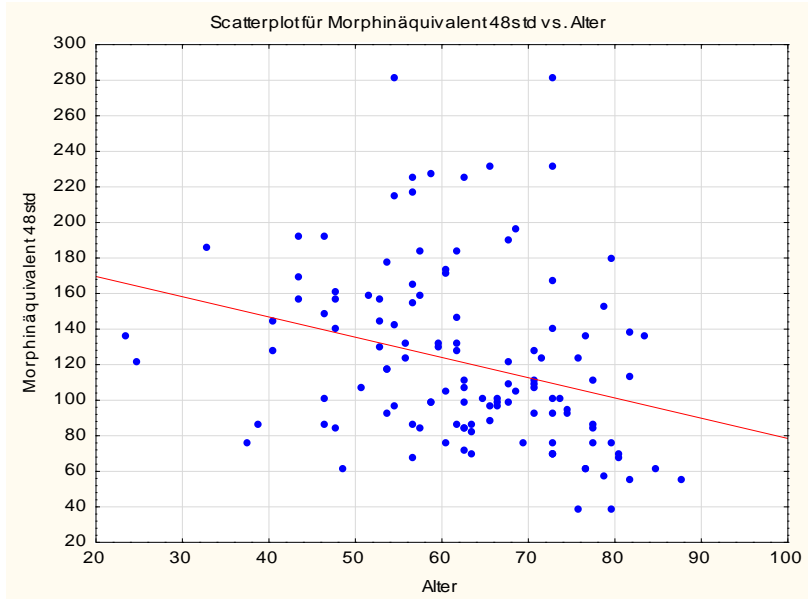

Abbildung 43: Scatterplot Morphinäquivalent $48 \mathrm{~h}$ vs. Alter

\subsubsection{Morphinverbrauch am 5. postoperativen Tag}

Das Morphinäquivalent am 5. postoperativen Tag weist eine deutliche Signifikanz zum MQS Opioide präoperativ auf. Für die multiple Regression wurde dieser deutlich bestehende Zusammenhang jedoch herausgenommen.

Es blieben aber noch 6 weitere signifikante Variablen. Sowohl der MPSS mit einem pWert von 0,002, die psychologischen Parameter KSI Hilfe und Hoffnungslosigkeit sowie der DASS-Depression als auch der WOMAC waren sowohl im Steifigkeitsscore als auch im Schmerzscore signifikant.

\subsection{Ausschlüsse von Variablen für die multiple Regression}

\subsubsection{MQS Opioide}

Obwohl der Opioidverbrauch präoperativ einen hohen Einfluss auf den postoperativen Gebrauch hat, haben wir die Variable aus den multiplen Regressionen herausgenommen, da $83,2 \%$ ( $n=104$ von 125) der Patienten gar keine Opioide präoperativ genommen haben und somit die Aussagekraft sehr vermindert ist.

Dennoch kann man an der Signifikanz von $p=0,007$ bei $48 \mathrm{~h}$ intra- bzw. postoperativem Morphinäquivalent und von $p=0,0001$ am 5. postoperativen Tag Morphinäquivalent 
erkennen, dass die Patienten, die vorher Opioide konsumiert haben auch postoperativ eine höheres Morphinäquivalent benötigen.

\subsubsection{MQS Nichtopioide}

Im Vergleich zum MQS Opioide haben deutlich mehr Patienten Analgetika und Coanalgetika präoperativ gegen die Schmerzen genommen. Dennoch haben auch hier $40 \%$ ( $n=50$ von 125) keine analgetischen Medikamente vor der Operation regelmäßig eingenommen.

Die Verteilung der übrigen $60 \%$ ist leider nicht sehr breit gestreut, sodass wir uns auch bei dieser Variable dagegen entschieden haben, sie in der multiplen Regression zu verwenden. Man kann jedoch bei der univariaten Regression des maximalen Schmerzes am 5. Tag eine Signifikanz von 0,04 errechnen.

\subsection{Multiple Regression}

Die signifikanten Variablen aus der explorativen univariaten Regression verwendeten wir nun im zweiten Schritt in einer rückwärtsläufigen multiplen linearen Regression, um möglichst neutral die Ergebnisse betrachten zu können. Die Ergebnisse sind in Tabelle 8 veranschaulicht. 
Tabelle 8: Aufstellung aller signifikanten univariaten Parameter für die multiple Regression signifikante Parameter hervorgehoben

\begin{tabular}{|l|l|l|l|l|}
\hline Variable & $\begin{array}{l}\text { Stärkster } \\
\text { Schmerz 1. pOP }\end{array}$ & $\begin{array}{l}\text { Stärkster } \\
\text { Schmerz 5. } \\
\text { pOP }\end{array}$ & $\begin{array}{l}\text { Morphinäquivalent } \\
\text { 48 Stunden }\end{array}$ & $\begin{array}{l}\text { Morphinäquivalent } \\
\text { 5. pOP }\end{array}$ \\
\hline Alter & n.s. & n.s. & 0,001 & n.s. \\
\hline BMI & 0,012 & 0,021 & n.s. & n.s. \\
\hline CPG & 0,008 & 0,041 & 0,02 & 0,048 \\
\hline MPSS & n.s. & 0,002 & n.s. & 0,002 \\
\hline painDETECT & n.s. & 0,000001 & n.s. & n.s. \\
\hline DASS-Depression & 0,001 & 0,003 & 0,005 & 0,01 \\
\hline DASS-Angst & 0,035 & 0,03 & n.s. & n.s. \\
\hline DASS-Stress & 0,002 & 0,005 & 0,033 & n.s. \\
\hline $\begin{array}{l}\text { KSI Hilfe und } \\
\text { Hoffnungs- } \\
\text { losigkeit }\end{array}$ & 0,003 & 0,001 & n.s. & 0,037 \\
\hline WOMAC-Schmerz & n.s. & n.s. & n.s. & 0,007 \\
\hline $\begin{array}{l}\text { WOMAC- } \\
\text { Steifigkeit }\end{array}$ & 0,044 & n.s. & n.s. & 0,03 \\
\hline WOMAC-Aktivität & 0,045 & n.s. & n.s. & n.s. \\
\hline $\begin{array}{l}\text { Durchs. Schmerz } \\
\text { letzte 3 Monate }\end{array}$ & 0,023 & 0,006 & n.s. & n.s. \\
\hline $\begin{array}{l}\text { Timed “Up and } \\
\text { Go"-Test }\end{array}$ & 0,027 & 0,043 & 0,037 & n.s. \\
\hline Treppengehtest & n.s. & 0,025 & n.s. & n.s. \\
\hline Schmerzschwelle & n.s. & 0,001 & 0,018 & n.s. \\
\hline
\end{tabular}




\subsubsection{Maximaler Schmerz postoperativ}

Bei der multiplen Regression stellten wir fest, dass der DASS-Stress allein unter den ganzen signifikanten Variablen die stärkste Aussagekraft hat (Abbildung 44). Er kommt auf ein modifiziertes $R^{2}$ von $13,8 \%$ und einen $p$-Wert von $<0,001$.

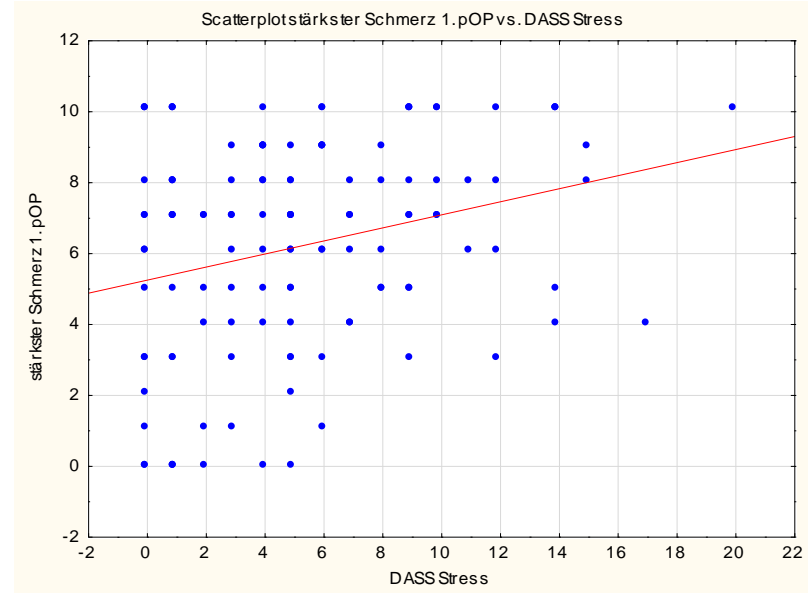

Abbildung 44: Scatterplot stärkster Schmerz 1. pOP vs. DASS-Stress

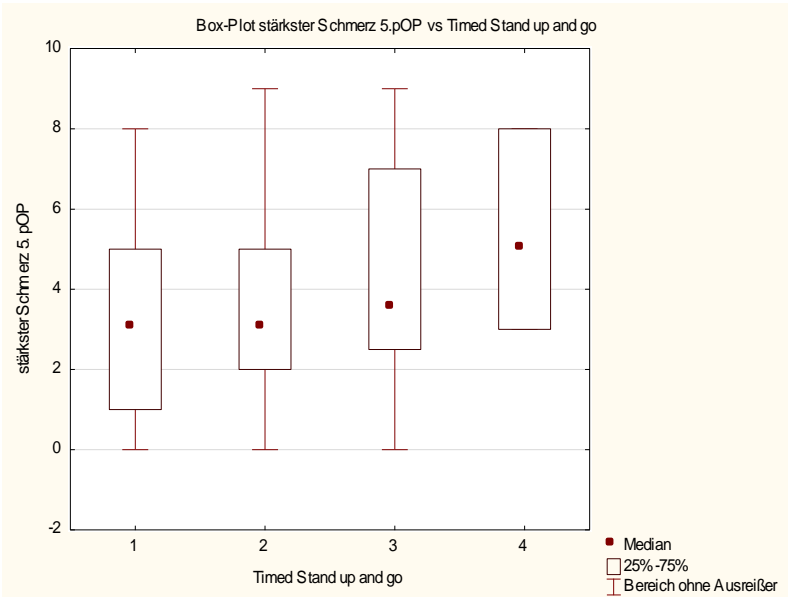

Abbildung 45: Boxplot stärkster Schmerz 5. pOP Tag vs. Timed "Up and Go"

Bei der Betrachtung des maximalen Schmerzes am 5. postoperativen Tag hatte der Timed „Up and Go“-Test (Abbildung 45) einen großen Einfluss und zusammen mit dem painDETECT die größte Aussagekraft. Diese Kombination kommt auf ein modifiziertes $R^{2}$ von $19,1 \%$ und einem $p$-Wert von $<0,001$. 


\subsubsection{Morphinäquivalent postoperativ}

Betrachtet man die Variablen, welche die stärkste Aussagekraft bei dem Morphinäquivalent nach 48 Stunden intra- und postoperativ haben, so weisen das Alter, der CPG und der DASS Depression das höchste modifizierte $\mathrm{R}^{2}$ mit $18.3 \%$ und einem p-Wert von 0,00002 auf.

Am 5. postoperativen Tag wird der Opioidverbrauch der Patienten jedoch am ehesten durch den MPSS und den WOMAC-Steifigkeitsscore beeinflusst. Insgesamt haben diese beiden Variablen ein modifiziertes $\mathrm{R}^{2}$ von $11,95 \%$ und einem $\mathrm{p}$-Wert von 0,002 .

\subsubsection{Tabellarische Darstellung der multiplen Regression}

Im Folgenden ist eine Aufstellung aller Variablen, welche nach der multiplen Regression die stärkste Aussagekraft aufweisen (Tabelle 9).

Tabelle 9: Ergebnisse der multiplen Regression

\begin{tabular}{|c|c|c|c|c|}
\hline Variable & $\begin{array}{l}\text { Stärkster } \\
\text { Schmerz 1. pOP }\end{array}$ & $\begin{array}{l}\text { Stärkster } \\
\text { Schmerz } 5 . \\
\text { pOP }\end{array}$ & $\begin{array}{l}\text { Morphinäquivalent } \\
48 \text { Stunden }\end{array}$ & $\begin{array}{l}\text { Morphinäquivalent } \\
\text { 5. pOP }\end{array}$ \\
\hline DASS-Stress & $\begin{array}{l}\text { Modifiziertes } \mathrm{R}^{2} \\
13,8 \%\end{array}$ & n.s. & n.s. & n.s. \\
\hline painDETECT & n.s. & \multirow{2}{*}{$\begin{array}{l}\text { Modifiziertes } R^{2} \\
19,1 \%\end{array}$} & n.s. & n.s. \\
\hline Timed „Up and Go & n.s. & & n.s. & n.s. \\
\hline Alter & n.s. & n.s. & \multirow{3}{*}{$\begin{array}{l}\text { Modifiziertes } \mathrm{R}^{2} \\
18,3 \%\end{array}$} & n.s. \\
\hline DASS-Depression & n.s. & n.s. & & n.s. \\
\hline CPG & n.s. & n.s. & & n.s. \\
\hline $\begin{array}{l}\text { WOMAC- } \\
\text { Steifigkeit }\end{array}$ & n.s. & n.s. & n.s. & \multirow{2}{*}{$\begin{array}{l}\text { Modifiziertes } \mathrm{R}^{2} \\
11,95 \%\end{array}$} \\
\hline MPSS & n.s. & n.s. & n.s. & \\
\hline
\end{tabular}




\section{Diskussion}

Es wurde eine prospektive Kohortenstudie über das Thema postoperativer Schmerz und Opioidverbrauch an primären Hüftgelenksersatzpatienten durchgeführt.

Hierbei wurden Patienten, welche sich einem total primären Hüftgelenksersatz unterzogen haben, am präoperativen Tag und wiederholt während des stationären Aufenthaltes postoperativ befragt und getestet. Dabei wurden physische, psychische und klinische Parameter erhoben, welche 1. univariat und dann 2. in der multiplen Regression, welche wir zur Detektion des vorhersagenden Parameters verwendeten, geprüft wurden. Man versucht folglich aus unterschiedlichen erhobenen Parametern die Konstellation bzw. den Parameter zu finden, welcher am ehesten eine Verbindung zu der festgelegten Zielvariable hat. Die Zielvariablen für diese Analysen sind in unserem Fall der stärkste postoperative Schmerz am 1. und 5. sowie das postoperative Morphinäquivalent innerhalb der ersten $48 \mathrm{~h}$ und am 5. postoperativen Tag.

Es zeigt sich, dass abhängig vom postoperativen Tag unterschiedliche Merkmale zum Tragen kamen.

Für die akuten postoperativen Schmerzen (1. und 5. Tag) sind in der univariaten Analyse präoperative Schmerzen, BMI, CPG, MPSS, painDETECT, DASS-Depression, DASSAngst, DASS-Stress, KSI Hilfe und Hoffnungslosigkeit, WOMAC-Steifigkeit, WOMACAktivität, Timed „Up and Go“-Test, Treppengehversuch, die Schmerzempfindlichkeit (Druckschmerzschwelle), der durchschnittliche Schmerz drei Monate vor der Operation und der präoperative Analgetikakonsum signifikant mit einer breiten Varianz. In der multiplen Regression zeigte sich letztlich für die Schmerzintensität am 1. pOP Tag nur noch der präoperative Stress und für den 5. pOP Tag der Timed „Up and Go“-Test sowie der painDETECT als signfikant.

Bei dem postoperativen Morphinäquivalent sind Alter, CPG, MPSS, DASS-Depression, DASS-Stress, KSI Hilfe und Hoffnungslosigkeit, WOMAC-Schmerz, WOMAC-Steifigkeit, der Funktionsparameter Timed „Up and Go“-Test sowie die Schmerzempfindlichkeit (Druckschmerzschwelle) und der präoperative Opioidkonsum signifikant. Auch hier besteht eine breite Varianz. In der multiplen Regression haben in den ersten 48 h, vom 
Morgen der Operation an gerechnet, das Alter, der CPG und die Depression die stärkste Aussagekraft. Am 5. pOP Tag sind es der MPSS zusammen mit der Steifigkeit im WOMAC.

Insgesamt muss man sagen, dass es keinen präoperativen Parameter gibt, der den akuten postoperativen Schmerz oder den postoperativen Opioidkonsum eindeutig beeinflusst bzw. vorhersagt.

Im Folgenden werden nun die signifikanten Parameter sowohl der univariaten als auch im Besonderen der multiplen Regression diskutiert. Hierbei bezieht sich diese Arbeit zunächst auf den Einfluss der präoperativen Scherzen und Analgetikaeinnahme des Patientenkollektivs. Darauf folgend werden die erhobenen Stammdaten zum Zeitpunkt der Aufnahme diskutiert und anschließend sowohl die körperlichen als auch die psychischen Prädiktoren erörtert. Im Anschluss wird der Bezug zum Annastift Hannover gestellt und zuletzt werden die Einschränkungen der Studie genannt.

\subsection{Allgemeines}

In dieser Studie liegt Osteoarthritis als Hauptindikation für einen primären Hüftgelenksersatz bei $77 \%$ der Fälle vor. Dies nähert sich zum Beispiel den Ergebnissen von Pivec et al. 2012, wo 90\% der operierten Parienten aufgrund einer Osteoarthritis operiert wurden. Die Zahlen bezüglich der Indikationen variiert jedoch von Land zu Land und innerhalb der ethnischen Herkunft (Merx et al. 2003).

Bezüglich der Schnitt-Naht-Zeit der Operation und der Anästhesieverfahren gibt es in dieser Studie keine Unterschiede und Auffälligkeiten, sodass diese nicht in die Berechnungen miteinbezogen wurde. Die längste Schnitt-Naht-Zeit beträgt 234 min und hebt sich damit als einzelner Wert vom restlichen Patientenkollektiv ab, wo die SchnittNaht-Zeit $\leq 200$ min gewesen ist.

\subsection{Präoperativer Morphin-, Analgetika- und Koanalgetikakonsum}

Der Konsum von Analgetika und Koanalgetika präoperativ beeinflusst den postoperativen stationären Verlauf in dieser Studie. Dies unterstreicht die Ergebnisse 
von Liu et al. 2012 aus den USA, wo der präoperative Gebrauch von Opioiden, Analgetika, Antikonvulsiva und Antidepressiva als Risikofaktoren für erhöhten akuten postoperativen Schmerz bei Hüft- und Knieendoprothesen unter anderen detektiert wurden. Jedoch ist zu berücksichtigen, dass lediglich $60 \%$ der Patienten präoperativ Analgetika und Koanalgetika eingenommen haben.

Der präoperative Opioidkonsum hat einen starken signifikanten Einfluss auf das Morphinäquivalent am 5. pOP Tag. Dies kann man eventuell auf eine leichte körperliche Abhängigkeit zurückführen, da der Körper schon vor der Operation sich an eine regelmäßige Einnahme von Opioiden gewöhnt hat bzw. ein Gewöhnungseffekt besteht und der Patient mehr Opioide toleriert. Insgesamt war unsere präoperativ konsumierende Patientengruppe zu klein, um sie in die multiple Regression miteinzubeziehen, da lediglich $18 \%$ überhaupt präoperativ Opioide eingenommen haben.

Insgesamt fällt bei unserem Patientenkollektiv auf, dass sich viele Patienten eine Hüftendoprothese einsetzen ließen, obwohl sie zuvor keine analgetische Medikation konsumiert haben. Dies könnte darauf zurückzuführen sein, dass für die Indikationstellung die funktionelle Einschränkung führend war, wie auch in der stark erniedrigten körperlichen SF-12 Summenskala deutlich wurde.

Perioperativ scheinen ausreichend Opiate mit der Standardanalgosedierung des Krankenhauses verabreicht worden sein, da die Patienten wenige Schmerzspitzen angaben und das Morphinäquivalent mit $60 \mathrm{mg} / \mathrm{d}$ einer mittleren Dosis entsprach (Paul et al. 2015) Bezüglich unerwünschter Nebenwirkungen gaben in unserem Patientenkollektiv $43,2 \%$ am 1. pOP Tag und 22,4 \% am 5. pOP Tag Emesis an.

Opioide haben viele Nebenwirkungen, die bei der Verabreichung bedacht werden müssen. So beeinträchtigen sie nicht nur den Gastrointestinaltrakt und die Blasenfunktion. Es wird diskutiert, ob Opioide auch die Heilung verzögern können, andererseits ist dies auch für starke Schmerzen der Fall. Eine immunsuppressive Wirkung wird ebenfalls diskutiert, ist aber bis heute nicht eindeutig nachgewiesen. Unter anderem erholen sich die Patienten schlechter in ihren Schlafphasen durch einen 
verminderten REM-Schlaf (Kehlet et al. 1996). Ziel sollte somit eine möglichst an Bedarf und Funktion angepasste und möglichst niedrige Dosierung sein.

Da sich aber Patienten gerade nach einer HTEP-Operation schnell wieder bewegen sollen, ist eine Kombinationstherapie mit NSAR und Opioiden besonders sinnvoll (Kehlet et al. 1996).

Generell ist es so, dass die Angst, eine Abhängigkeit von Opioiden zu entwickeln, besteht (Huang et al. 2001). Anhand unserer Daten können wir lediglich bestätigen, dass die Werte der Patienten, welche postoperativ stärkere Schmerzen angeben und demzufolge ggf. mehr Analgetika benötigen, mit psychologischen Tests wie KSI Hilfe und Hoffnungslosigkeit sowie DASS-Depression und -Stress deutlich korrelieren. Ob eine intensivere Aufklärung demnach anzuraten ist, um mögliche Ängste zu lindern, da Ängste die Genesung beeinträchtigen und das Vertrauen in den Erfolg der medizinischen Maßnahmen abschwächen, bleibt zu vermuten. Da am 1. und 5. postoperativen Tag bei unserem Kollektiv eine Patientenzufriedenheit mit durchschnittlich 12 von möglichen 15 Punkten angegeben wurde, fühlten sich die Patienten anscheinend im Durchschnitt gut informiert. Dennoch ist die Patientenzufriedenheit verbesserungsfähig.

\subsection{Präoperativer Schmerz}

Wie bereits eingangs erwähnt, haben präoperative Schmerzen einen großen Einfluss auf postoperative Schmerzen in der frühen Phase (Kalkman et al. 2003; Macrae 2001; Liu et al. 2012). Das betrifft in dieser Studie sowohl den ersten postoperativen Tag als auch deutlich stärker den 5. postoperativen Tag. Die Patienten, welche vor der Operation bereits an stärkeren Schmerzen litten, gaben auch nach der Operation deutlich stärkere Schmerzen an.

Neben den Parametern, die präoperativ selbst Schmerzen abgefragt haben, wie der CPG, der painDETECT und der WOMAC-Schmerz, gibt es noch hohe Korrelationen zur Aktivität und zur Hilfe und Hoffnungslosigkeit. 
In jedem Fall sollten die postoperativen Schmerzen gemindert werden, da mittlere bis starke postoperative Schmerzen das Outcome und die Patientenzufriedenheit beeinflussen. Schmerzen verursachen Probleme beim Mobilisieren und Laufen. Zusätzlich sorgen nächtliche Schmerzen für einen unruhigen, oder gar verminderten Schlaf. Insgesamt betrifft das bei den HTEP Patienten $47 \%$, welche am ersten postoperativen Tag unter starken Schmerzen litten (Wylde et al. 2011 a). In unserem Patientenkollektiv gaben 40,8 \% der Patienten an, am ersten, und $22,8 \%$ der Patienten am 5. Postoperativen Tag in der vorherigen Nacht ungewollt erwacht zu sein. Doch nur $12,8 \%$ der Patienten hätten am ersten Tag und $4 \%$ am 5. postoperativen Tag gerne mehr Schmerzmittel erhalten. Der Schmerz ist nicht als die einzige Ursache eines unruhigen Schlafes zu betrachten, hat aber scheinbar auch einen Einfluss auf den Schlaf (Chouchou et al. 2014). Vielmehr wird es eine ungewohnte Schlafumgebung, Geräusche und Schlafposition nach einer solchen Operation sein, die den Organismus beeinflussen. Dennoch kann man bereits anhand dieser Daten erkennen, dass das angewandte Schmerzkonzept im Annastift Hannover bei den meisten HTEP-Patienten anscheinend subjektiv und objektiv ausreicht.

Um die chronischen Schmerzpatienten vor der Operation bereits zu erkennen und herauszufinden, welche Merkmalsgruppen sie haben und ob die Klassifizierungen vorhersagen können, wie stark die Beeinträchtigung nach der Operation sind, wurde der MPSS, der CPG sowie der painDETECT genauer betrachtet, da gerade diese drei Parameter einen signifikanten Bezug aufwiesen.

\subsubsection{MPSS}

Wie bei Hampel und Moergel 2009 befanden sich im Stadium III vermehrt Frauen. Dies kann daran liegen, dass in dieser Studie auch vermehrt Frauen eingeschlossen wurden, da diese häufiger einen Hüftgelenksersatz bekommen. Dennoch ist die Differenz in diesem Stadium zwischen Männern und Frauen stärker ausgeprägt als in den beiden anderen Stadien. Diese Konstellation weist darauf hin, dass sich Frauen vermehrt erst in einem erhöhten Chronifizierungsstadium des Schmerzes einer Operation unterziehen. 
Generell lässt sich sagen, dass ein Zusammenhang des MPSS zum Analgetikakonsum prä-und postoperativ klar zu erkennen ist. Da der MPSS selbst die Medikamentenhistorie der Patienten erfragt, liegt dies nahe. Jedoch bezieht sich der MPSS nicht nur auf die Menge der Analgetika, sondern auch auf mögliche Entzugsbehandlungen und die Regelmäßigkeit der Einnahme.

Die Aktivitätsvariable des WOMAC-Scores hingegen ist sehr interessant in Bezug zum MPSS. Obwohl der MPSS die Bewegungsfähigkeit nicht erfragt, scheint die subjektive körperliche Aktivität der Patienten in einem direkten Zusammenhang mit diesem Test zu stehen, da sie in einer erhöhten Korrelation zueinander stehen.

Depressionen sowie Hilfe und Hoffnungslosigkeit haben einen Einfluss auf die MPSSKlassifizierung. Diese sind jedoch nicht signifikant. Wie Hampel und Moergel 2009 bereits anregten, ist eine psychometrische Diagnostik bei einem auffälligen MPSS zu empfehlen.

In der Literatur gibt es verschiedene Resultate, ob man mit dem MPSS auch die Prognose chronischer Schmerzen vorhersagen kann. Hüppe et al. 2011 weisen darauf hin, dass es auf die erwählten Outcomeparameter ankommt. In dieser Studie hat der MPSS am 5. pOP Tag für beide Outcome-Parameter stark signifikante Werte univariat und ist in der multiplen Regression des Morphinäquivalentes am 5. postoperativen Tag zudem gemeinsam mit der Steifigkeit der Parameter mit der stärksten Aussagekraft. Demnach kann man anhand dieser Ergebnisse sagen, dass der MPSS auf eine verzögerte Rekonvaleszenz hindeutet. Indirekt kommt auch der Analgetikakonsum zum Ausdruck.

Das Problem beim MPSS ist, dass man seine Gruppenzuordnung nicht mehr verbessern kann. Liegt eine schmerzbedingte Krankheit schon mehrere Jahre zurück und hat keinen Einfluss mehr auf die akute Situation, so kann der Patient als chronifiziert eingestuft werden, obwohl es in dem aktuellen Zustand eventuell keine Chronifizierung mehr gibt. Umzüge und damit verbundene Arztwechsel, sowie die schmerzbedingten Operationen durch beispielsweise Sportunfälle oder Unfälle in der Kindheit werden nicht 
berücksichtigt. Es liegt also an dem behandelnden Arzt das Ausmaß der Einstufung zu interpretieren.

\subsubsection{CPG}

Obwohl der CPG ursprünglich für Rückenschmerz-, Kopfschmerz- und Temporomandibularschmerz-Patienten entwickelt wurde, ist er bereits für allgemeine Schmerzpatienten validiert worden (Smith et al. 1997).

Da der Chronic Pain Grade nicht nur die Schmerzintensität, was den signifikanten Einfluss der präoperativen Schmerzen erklärt, sondern auch die Beeinträchtigung der Befragten berücksichtigt, haben alle Funktionsparameter einen signifikanten Einfluss auf die Schweregradeinteilung. Gerade die WOMAC-Scores Schmerz und Aktivität sowie der Timed „Up and Go“-Test weisen eine deutlich erhöhte Korrelation zu diesem Klassifikationssystem auf. Auch in der Primärliteratur des CPG wird schon ein Zusammenhang mit der schmerzbezogenen Beweglichkeit festgestellt (von Korff et al. 1992).

Obwohl der CPG sich auf die funktionelle Beeinträchtigung beschränkt, stehen die psychologischen Parameter wie Angst, Depression, Stress, Hilfe und Hoffnungslosigkeit, Katastrophisierung, Durchhalteappell und die schmerzbezogene Angst in einem starken Zusammenhang zu dieser Klassifizierung.

Das auch der painDETECT, der eigentlich den neuropathischen Schmerz identifiziert, einen großen Einfluss auf den CPG hat, ist sehr interessant, gerade weil nicht die Schmerzintensität, sondern der Schmerzcharakter beim painDETECT in den Score miteinfließt.

Auch der BMI, ein Parameter, der die postoperativen Schmerzen signifikant beeinflusst, ist bei diesem Schmerzchronifizierungseinteilung des CPG signifikant.

Vergleicht man nun den CPG mit dem MPSS als Prädiktor für akute postoperative Schmerzen oder akuten postoperativen Opioidverbrauch, so ist der MPSS als prädiktiver Faktor in den multiplen Regressionen am 5. postoperativen Tag sowohl beim 
Opioidkonsum als auch bei der stärksten Schmerzangabe identifiziert worden. Ob dies auch für die langfristige Prognose gilt, wurde in dieser Doktorarbeit nicht untersucht. Der CPG hingegen ist nur als eine Variable der stärksten postoperativen Schmerzen am 1. Tag signifikant. Dennoch ist bei dem CPG zu berücksichtigen, dass diese Graduierung auch deutlicher die Beweglichkeit miteinbezieht.

\subsection{3 painDETECT}

Wie bereits zu Beginn der Diskussion erwähnt und im Ergebnisteil errechnet, weist der präoperativ erhobene painDETECT eine starke Korrelation und Signifikanz zu den akuten postoperativen Schmerzen am 5. postoperativ auf.

Der painDETECT bezieht sich auf den Schmerzcharakter und neuropathische Schmerzen und ermittelt dadurch eine mögliche nervale Beteiligung. An und für sich sollte bei einer Coxarthrose, welche die Hauptursache einer primären Hüftendoprothese in dieser Studie war, wenig nervale Beteiligungen auftreten, jedoch sind dadurch Patienten mit unterschiedlichen Schmerzlokalisationen und -ursachen identifizierbar.

Demzufolge sind Patienten, die eine neuropathische Schmerzkomponente aufweisen, ggf. mit anderen analgetischen Medikamenten besser $\mathrm{zu}$ behandeln als mit dem orthopädischen Standardschema NSAR plus Opiat (Mallick-Searle et al. 2016).

Eine mögliche andere Betrachtungsweise wäre eine präoperativ erhöhte Schmerzempfindlichkeit, welche postoperativ mit erhöhten Schmerzen einhergeht, wobei der painDETECT nur teilweise mit der erhobenen Schmerzschwelle korreliert.

Insgesamt hatten nur 2 Patienten einen Summenscore, welche für einen neuropathischen Schmerz spricht, sodass die Aussagekraft des Parameters deutlich vermindert ist. Möglichweise sind dies Probanden mit Zeichen einer zentralen Sensibilisierung, einer zusätzlichen schmerzhaften Polyneuropathie oder Fibromyalgie gewesen (Koroschetz et al. 2011). Da Nebenerkrankungen in dieser Studie nicht berücksichtigt wurden, bleibt diese Vermutung jedoch spekulativ. 
Der präoperative neuropathische Schmerzcharakter, gemessen mit dem painDETECT, steht in Verbindung zum durchschnittlichen Schmerz 3 Monate vor der Operation, dem Alter und den präoperativ erhobenen psychologischen Parametern. Diese Parameter sind in der Studie von Ip et al. 2009 als die aussagestärksten Variablen detektiert worden. Warum gerade dieser Parameter sowohl univariat als auch multivariat mit dem Funktionsparameter Timed „Up an Go“-Test die stärkste Aussagekraft hat, ist schwer zu sagen. Schäfer et al. beschrieb 2017, dass sich Patienten mit neuropathischen Schmerzen eher auf den Schmerzlokus fokussieren, welches am ehesten auch auf Patientenkollektiv zutrifft, wohingegen Patienten mit zentralen Schmerzen eher psychisch involviert sind. Des Weiteren stellte Shaygan et al. 2017 fest, dass jeder Schmerz eine neuropathische Komponente hat und gerade jene Patienten, welche eine hohe Schmerzintensität oder funktionelle Einschränkung haben, vermehrt neuropathische Schmerzen erleiden. Eventuell triggert eine neuropathische Schmerzkomponente die Schmerzempfindung postoperativ.

\subsection{Stammdaten als Prädiktor}

\subsubsection{BMI}

Laut der DEGS1-Studie liegt die hier untersuchte Patientenklientel mit dem BMI nur geringfügig unter dem Durchschnitt der Frauen in Deutschland, welche zwischen dem 60 und 69 LJ bei 27,9 liegt. Bei den Männern mit 28,1 spiegelt der Wert exakt den Durchschnittswert in diesem Patientenkollektiv wider (Mensink et al. 2013).

Aus dem vorhandenen Datensatz kann man sagen, dass ein höherer BMI mit stärkeren akuten postoperativen Schmerzen einhergeht, was die Ergebnisse von Chung et al. 1997, und Liu et al. 2012, bestätigt. Eine mögliche Erklärung wäre, dass sich übergewichtige Personen weniger bewegen können/wollen (Busato et al. 2008) und so den Fokus auf die Schmerzen erhöhen. Ein anderes Erklärungsmodell wäre die Tatsache, dass Patienten mit einem höheren BMl auch mit ihrer neuen Hüfte mehr Gewicht tragen müssen, als Patienten mit einem niedrigeren BMI. Dieser zusätzliche Druck könnte dafür sorgen, dass vermehrt Schmerzen ausgelöst werden. Eine weitere Betrachtungsweise wäre die Vermutung, dass die Patienten im Verhältnis zum 
Körpergewicht weniger Analgetika erhalten haben, da sie trotz stärkerer Schmerzen im Mittel gleichviel Morphinäquivalent bekommen haben. Da die Dosis nicht an das reelle Körpergewicht, sondern einem Idealkörpergewicht angepasst wird, ist dies durchaus korrekt durchgeführt. Jedoch verteilen sich die Substanzen möglicherweise anders im Körper.

Kessler und Käfer stellten in ihrer Veröffentlichung 2007 jedoch deutlich dar, dass weder Übergewichtigkeit noch Adipositas das Outcome von Patienten beeinflussen. Als Parameter benutzten sie den WOMAC sowohl zehn Tage als auch drei Monate nach der Operation sowie die Zeit des stationären Aufenthaltes. Die Ergebnisse widersprechen ein wenig den Resultaten dieser Studie, da hier der BMI zumindest Einfluss auf die postoperative Schmerzwahrnehmung am 5. pOP Tag nimmt. Ob sich diese Schmerzdifferenz in den folgenden Tagen noch gehalten hätte, kann man mit dieser Datenauswertung nicht sagen. Da in der Studie von Kessler und Käfer 2007 der WOMAC-Schmerz als Endpunkt genommen wurde, kann auf diesem Weg der Vergleich zusätzlich erschwert sein.

Laut Batsis et al. 2009 steht ein erhöhter BMI in hoher Korrelation mit der OP-Dauer. Bei Peters et al. 2007 wird dieser Einfluss auf postoperative Schmerzverläufe aber erst nach über 3 Stunden sichtbar. Da in dieser Studie lediglich eine Operation mit 3,9 Stunden so lang gedauert hat, kann man für unsere Untersuchung nachvollziehbar keinen Zusammenhang identifizieren.

\subsubsection{Alter}

Die Aussage von Liu et al. 2012, dass jüngere Patienten postoperativ nach einer HTEP Operation mehr Schmerzen angeben als ältere Patienten, kann man in diesem Patientenkollektiv nicht bestätigen. Wenn man bei diesem Patientenkollektiv die Schmerzangaben postoperativ betrachtete, so kann man bei den Altersklassen keine relevanten Unterschiede erkennen. In dieser Studie wurden allerdings auch vermehrt ältere Patienten operiert, da ein elektiver Hüftgelenksersatz erst ab dem 60 LJ empfohlen wird (Innocenti et al. 2013). 
Da ein erhöhtes Morphinäquivalent nicht mit erhöhten Schmerzen am ersten postoperativen Tag einhergeht, kann man einen Fehlgebrauch eigentlich ausschließen. Diese Ergebnisse entsprechend den Daten von Petre et al. 2012, wo zusätzlich angemerkt wurde, das ältere Patienten auch mehr opioidabhängige Nebenwirkungen haben. Dies war bei unserem Patientenkollektiv nicht der Fall. Die Korrelationen zur postoperativen Nausea beträgt nur 0,04 am 1. und 0,05 am 5. pOP Tag und Emesis am 1. pOP 0,109 und am 5. pOP 0,057 .

Beim Morphinäquivalent peri- bzw. postoperativ in den ersten 48 Stunden haben nicht nur das Alter und das Geschlecht einen großen Einfluss auf den Gebrauch, sondern auch die psychologische Depressionskomponente. Das bestätigt vorherige Veröffentlichungen, welche das Alter und die psychologische Beeinträchtigung als Prädiktoren für den Analgetikakonsum ermittelten (Ip et al. 2009).

Nilsdotter et al. 2003, gab an, dass das Alter ein negativer Prädiktor für das postoperative Gesamtergebnis ist und dies nicht an der Anzahl der Komorbiditäten liegt. Was den stationären akuten postoperativen Verlauf bezüglich der Schmerzen und des Analgetikakonsums betrifft, so kann man dieses Ergebnis nicht bestätigen. Was aber den langfristigen Verlauf betrifft, so kann dies in zukünftigen Analysen dieser Studie berücksichtigt werden.

\subsubsection{Geschlechtsspezifische Unterschiede}

Das Geschlecht war zu keinem Erhebungszeitpunkt weder für die postoperativen Schmerzen noch für das Morphinäquivalent ein signifikanter Faktor. Dennoch gibt es interessante Ergebnisse in Bezug auf die Ausgangslage der Patientinnen und Patienten.

Frauen haben ein erhöhtes Risiko für akute postoperative Schmerzen nach einer HTEP (Liu et al. 2012). Ob Frauen wirklich stärkere Schmerzen aufgrund einer intensiveren Wahrnehmung haben oder die Schmerzen tatsächlich objektiv stärker sind, ist schwer zu sagen. In der Literatur findet man in vielen Studien (Etherton et al. 2014, Fillingim et al. 2009) höhere Schmerzangaben beim weiblichen Patientenkollektiv. Zudem gibt es auch verschiedene Theorien und Ergebnisse in der Literatur, welche versuchen, die erhöhten Schmerzangaben zu erklären. So haben Frauen wohl geringere endogene 
Schmerzhemmsysteme, die Opioidrezeptoren scheinen zudem östrogensensibel zu sein. Außerdem können Frauen multiple Schmerzen wahrnehmen und geben auch den Schmerz schneller an. Es gibt noch viele weitere Erklärungen, warum Frauen Schmerz anders wahrnehmen, aber auch bei den psychischen Faktoren sind Frauen häufiger betroffen. Zum Beispiel haben sie eine höhere Depressionsrate (für eine Übersichtsarbeit siehe LeResche 2011)

Anhand der Schmerzschwellenmessung kann man jedenfalls erkennen, dass Frauen in Übereinstimmung mit vielen vorausgegangenen Untersuchungen eine niedrigere Druckschmerzschwelle angeben als Männer (Fillingim et al. 2009). Eine Einschränkung für die aktuelle Untersuchung ist die Tatsache, dass beide Untersucher weiblich waren. Es ist bekannt, dass männliche Probanden höhere Schmerzschwellen angeben, wenn sie von einer Frau untersucht wurden. Der Unterschied zwischen Männern und Frauen könnte in dieser Studie somit noch verstärkt worden sein. Dagegen spricht, dass die Schmerzschwelle für das gesamte Kollektiv mit dem Maximalschmerz am 5. postoperativ Tag und dem Opiatverbrauch in den ersten $48 \mathrm{~h}$ korrelierte.

Allerdings werden auch im Durchschnitt stärkere Schmerzen prä- und postoperativ bei unserem Patientenkollektiv von den Frauen angegeben. Zusammen mit der niedrigeren Druckschmerzschwelle würde dies die These bestätigen, dass Frauen Schmerz schneller wahrnehmen. Ob man aus diesem Grunde Frauen auch intensiver schmerztherapeutisch behandeln sollte, ist daraus nicht abzuleiten. Zumindest ist zu berücksichtigen, dass mit einer höheren Dosierung der Schmerzmedikation die Intensität der Nebenwirkungen ansteigt. Des Weiteren widerspricht diese Studie auch vorherigen Studien wie z. B. Ip et al., 2009, wo es keinen geschlechtsspezifischen Unterschied gibt.

Die erhöhten Schmerzangaben unseres weiblichen Patientenkollektives würden allerdings auch für die Theorie sprechen, dass Frauen erst in einem späteren Stadium zum Spezialisten gehen, um sich einer HTEP zu unterziehen (Jüni et al. 2010). Diese These wird unterstützt von den Ergebnissen aus der Studie von Lavernia et al. 2011, in der feststellt wurde, dass sich Frauen erst in einem fortgeschrittenerem Stadium einer HTEP unterziehen und ihre Beeinträchtigung und die Schmerzen scheinbar besser bzw. 
länger tolerieren als Männer. Im Verlauf verhalten sich Männer und Frauen aber scheinbar gleich bzw. geben die Frauen einen besseren Allgemeinzustand an. Ob die anatomisch unterschiedliche Acetabulumform und der "femoral offset“ oder der Abstand vom Mittelpunkt der Rotation des Femurkopfes bis zur Halbierungslinie der Längsachse des Femurs (Lecerf et al. 2009; Atkinson et al. 2010) bei der Frau durch eine andersartige Belastung einen Einfluss auf primäre Osteoarthritis und Schmerzen hat, lässt sich nur vermuten (Merle et al. 2014). Es ist jedoch auffällig, dass Frauen im Durchschnitt dieser Studie fast 7 Jahre früher operiert wurden, als Männer.

In diesem Patientenkollektiv konnte man in der uni- und multivariaten Analyse keinen signifikanten Einfluss auf die postoperativen Endpunkte Morphinäquivalent oder Schmerzempfindung detektieren. Weitere Studien zeigen ebenfalls keinen signifikanten Zusammenhang mit dem Geschlecht (Ip et al. 2009).

\subsection{Körperliche Prädiktoren}

Lavernia et al. beschrieben 2009, dass Patienten, welche präoperativ bereits funktionell eingeschränkt waren, postoperativ ein schlechteres Ergebnis aufwiesen. Dabei erholen sich die stärker betroffenen Patienten im ersten Jahr zwar schneller als die anderen, erreichen jedoch nicht die Funktionsfähigkeit der Patienten, die präoperativ eine bessere Funktion hatten.

Diese Feststellung kann man mit diesem Datensatz nicht gänzlich bestätigen, da in dieser Doktorarbeit eine Verlaufserhebung des WOMACs nicht betrachtet wird und sich diese Arbeit nur auf die erste postoperative Woche bezieht. Anhand der erhöhten Schmerzen am ersten postoperativen Tag und eines erhöhten Morphinäquivalentkonsums am fünften postoperativen Tag können wir jedoch eine stärkere Beeinträchtigung für die betroffenen Patienten feststellen. In der multiplen Regression ist der WOMAC-Steifigkeit zusammen mit dem MPSS der Parameter mit der stärksten Aussagekraft am 5. postoperativen Tag beim Morphinäquivalent. Warum allerdings der Funktionsteil des WOMACs lediglich starke Korrelationen, jedoch keine Signifikanz aufweist, bleibt spekulativ, ist aber vermutlich durch die hohe Interkorrelation der WOMAC-Subskalen erklärt. 
Die präoperative körperliche Funktion gemessen mit dem Timed „Up and Go“-Test und die Mobilität u.a. auch gegen die Schwerkraft, festgestellt mit dem Treppengehversuch, dienen in der multiplen Regression nur teilweise als signifikante Prädiktoren für postoperative Schmerzen. Insgesamt kann man anhand des signifikanten Einflusses des Timed „Up an Go“-Tests auf den 5. postoperativen Tag auf die Schmerzen demnach die Aussage von Schneider et al. 2009 in der akuten postoperativen Phase bestätigen, dass die präoperative Funktionalität als Prädiktor für postoperative Schmerzen gilt. Laut dieser Veröffentlichung gaben Patienten mit einer guten präoperativen Funktionalität postoperativ weniger Schmerzen an.

In dieser Studie ist die Druckschmerzschwelle sowohl für die postoperativen Schmerzen am 5. postoperativen Tag als auch für das Morphinäquivalent nach $48 \mathrm{~h}$ univariat signifikant. Da sowohl männliche als auch weibliche Patienten in dieser Studie betrachtet werden, kann man anhand dieser Daten womöglich einen Schritt weitergehen als Abrishami et al. 2011, welche in ihrem Review feststellten, dass die Schmerzschwelle ein Prädiktor für die Stärke des postoperativen weiblichen Schmerzempfindens ist. Ein weiterer Aspekt wäre, statt der generellen durchschnittlichen Schmerzempfindlichkeit einen Einfluss der lokalen und seitenbezogenen Schmerzempfindlichkeit in zukünftigen Studien zu analysieren.

Die gemessene Schmerzschwelle präoperativ ist also ein Hinweis für beide Geschlechter stärkere postoperative Schmerzen zu empfinden bzw. anzugeben.

\subsection{Psychische Prädiktoren}

Es gibt viele psychologische Einflüsse auf den Morphingebrauch und die Schmerzen. In dieser Studie fielen besonders die Parameter Stress, Depression, Angst sowie Hilfe und Hoffnungslosigkeit auf, welche in einem starken Zusammenhang miteinander stehen (Ip et al. 2009; Singh und Lewallen 2010; Bener et al. 2013; Nilges und Essau 2015).

Patienten mit einem erhöhten Depressions- und Angstscore präoperativ geben postoperativ während des stationären Aufenthaltes stärkere Schmerzen an (Ene et al. 2006). Diese Feststellung ließ sich mit diesem Patientenkollektiv durchaus bestätigen. 
Univariat haben diese beiden Faktoren signifikante p-Werte sowohl am ersten als auch am fünften postoperativen Tag. Riediger et al. 2010, spricht von einem schlechteren Ergebnis bei depressiven Patienten 6 Wochen nach der Operation. Da Schmerzen das operative Ergebnis stark beeinflussen, ist diese Tendenz auch schon an den Werten dieser Arbeit erkennbar.

2 Jahren postoperativ gilt Depression auch als Prädiktor für den Opioidkonsum (Singh und Lewallen 2010). Mit diesem Datensatz kann man des Weiteren sagen, dass dieser Einfluss bereits beim stationären Aufenthalt direkt nach der Operation zu sehen ist, da sowohl am ersten als auch am fünften postoperativen Tag der Depressionsparameter univariat signifikant mit dem Morphinäquivalent korreliert. Jedoch muss berücksichtigt werden, dass nur eine geringe Anzahl dieses Patientenkollektivs bezüglich der Depression klinisch relevante Grenzwerte überschritten.

Unter hoch chronifizierten Schmerzpatienten, zu denen dieses Patientenkollektiv nach den Auswertungen nicht zählt, findet man häufig milde Ausprägungen von Depressivität. Das Problem bei diesen Patienten ist häufig, dass sie sich aus vielen Bereichen ihres Lebens zurückziehen, was ein Teil des Krankheitsbildes ist. Dies führt zu einer Inaktivierung sowohl sozial als auch physisch. Da die Muskeln demzufolge weniger genutzt werden, atrophieren sie. Dies führt bei Belastungen zu starken Schmerzen, was wiederum den Rückzug fördert (Hasenbring et al. 2001).

Es gibt die Theorie, dass Angst die Heilung verzögert (Munafo und Stevenson 2001). Angst sorgt bei manchen Patienten jedenfalls dafür, dass sie Schonhaltungen einnehmen und Schonbewegungen durchführen, die bereits durch einen leichten Schmerzreiz ausgelöst werden (Hasenbring et al. 2001). Solange diese Mechanismen nur kurzfristig während der Heilungszeit genutzt werden, hat sowohl der Schmerz als auch die Angst, sich weiter zu verletzen, eine natürliche Funktion und dient der Genesung. Allerdings wird dieses Empfinden zu einem Problem, wenn die Angst trotz Heilung bestehen bleibt und es zu einem ausgeprägten Vermeidungsverhalten kommt. So stellten Vlaeyen et al. 1995 fest, dass die Angst vor Bewegungen nicht von der Stärke des erlebten Schmerzes abhängig ist. Für das in dieser Studie untersuchte 
Kollektiv konnten wir solche Zusammenhänge nicht sehen, da sie TSK keine relevanten Zusammenhänge mit den gewählten Endpunkten aufwies.

Unabhängig von Überlegungen zum Mechanismus zeigten andere Untersuchungen, dass der präoperativ festgestellte ängstliche Gemütszustand allein bereits als Prädiktor für postoperative Schmerzen (Katz et al. 2005, Ip et al. 2009).

Ein Mittel zur Entdeckung solcher Patienten und auch eine entsprechende präoperative Behandlung zur Angstreduktion werden daher empfohlen (Kain et al. 2000).

Allerdings muss man bei unserem Patientenkollektiv anmerken, dass körperliche Symptome, nach denen im DASS-Angst-Fragebogen gefragt werden, bei älteren Personen häufiger mit oder ohne Angst vorkommen, als bei jüngeren (Wood et al. 2010). Dies ist zum Beispiel bei den Bias „ich zittere“, „ich hatte Atemprobleme“, „ich habe meinen Herzschlag gespürt, ohne dass ich mich körperlich angestrengt habe“. Zudem kann ein „trockener Mund“ auch eine Nebenwirkung des Opioidkonsums oder auch ein Symptom der Exsikkose sein, welche bei älteren Menschen häufiger auftritt, als bei jüngeren.

Bei dem hier untersuchten Patientenkollektiv hatte der präoperative Stress die stärkste Aussagekraft bezüglich der postoperativen Schmerzen einen Tag nach der Operation. Es gibt eine Studie, die besagt, dass Menschen mit erhöhtem Stress, sowohl beruflich als auch privat, häufiger Schmerzen angeben (Bener et al. 2013).

Chronisch gestresste Personen, welche verstärkt auf eine erneute Stresssituation, wie beispielsweise eine Operation, reagieren, haben auch eine erniedrigte Chance auf ein gutes chirurgisches Ergebnis (Geiss et al. 2005). Warum in unserem Fall aber die Schmerzen bereits am ersten postoperativen Tag erhöht waren, ist damit nicht erklärt.

Anscheinend sorgt eine neue Stresssituation auf einen bereits gestressten Organismus anders als bei weniger gestressten Personen. Eine Überlegung wäre, dass der Patient sein Ziel, die Operation, nun hinter sich hat und der Organismus zur Ruhe kommen kann, da viele Stressoren im Krankenhausumfeld zunächst in den Hintergrund treten und andere hingegen hervortreten. Der Körper hat also Zeit und Energie seine 
Wahrnehmung zu verändern und kann somit unter anderem den Schmerz viel deutlicher spüren. Zuvor war die Aufgabe des Körpers zu funktionieren und die Stresssituationen zu bewältigen und sich möglichst nicht von körperlichen Symptomen daran hindern zu lassen.

Möglicherweise liegt es aber daran, dass eine gestresste Person eine höhere Erwartung an eine Operation hat und mit der postoperativen Situation ganz anders umgeht.

Eine andere Überlegung wäre, dass vermindertes postoperatives Cortisol einen Einfluss auf die Schmerzempfindung hat. Da in unserem Fall die Variable Stress aber nicht mit dem Cortisolspiegel gemessen wurde, sondern der Fragebogen sich vielmehr auf den subjektiven Stress des Patienten fokussiert, ist das schwer zu vergleichen (Geiss et al. 2005; Lautenbacher et al. 2009).

Besonders die psychologischen Parameter Depression sowie Hilfe und Hoffnungslosigkeit stehen in einem besonders starken Zusammenhang zum Stress.

Wood et al. stellten 2010 fest, dass Personen unter 60 LJ einen höheren Depressionsund Stressscore haben. Diese Werte sind bei diesem Datensatz nicht signifikant zu bestätigen.

In dieser Studie konnten wir keinen Einfluss von Anzeichen einer Somatisierung, erfasst durch den $\mathrm{PHQ}$, präoperativ auf den postoperativen Verlauf feststellen. Dies ist möglicherweise auf die Tatsache zurückzuführen, dass nur wenig Patienten überhaupt einen erhöhten Score hatten und keine gute Verteilung der Werte vorhanden war. Dieses Ergebnis widerspricht etwas den Resultaten von Riediger et al. 2010, da dort ein Einfluss von einer Somatisierung auf den postoperativen Verlauf, insbesondere jedoch auf das operative Ergebnis nach 6 Wochen erkennbar war. Da sich diese Arbeit jedoch nur auf die erster postoperative Woche beschränkt, kann dies ggf. in weiteren Arbeiten diskutiert werden.

Eine Bemerkung grundsätzlicher Art wäre noch, dass sich diese Assoziationen zwischen postoperativem Schmerz und Depression/Stress in einem klinisch meist unauffälligen 
Bereich der Fragebögen bewegen. Das stellt die Frage nach den optimalen Fragebögen (ganz einfach versus kompliziert), um diese Grauzone besser zu charakterisieren.

\subsubsection{Schmerzchronifizierungsmodelle}

Wie bereits eingangs erwähnt, gibt es verschiedene Schmerzchronifizierungsmodelle.

In dieser Studie ist das Katastrophisieren gemessen mit dem KSI kein signifikanter Faktor. Weder bei den Patienten mit chronischem Schmerz präoperativ, noch beim postoperativen Schmerzverlauf und Analgetikakonsum. Die Variable korreliert aber besonders stark mit der bewegungsbezogenen Angst, welche wir mit dem TSK gemessen haben. Weitere Korrelationen kann man dort mit dem DASS-Stress und den beiden ergänzenden Scores aus dem KSI, Durchhalteappell und Hilfe- und Hoffnungslosigkeit erkennen.

Es mag also sein, dass Katastrophisieren langfristig gesehen einen starken Einfluss auf Chronifizierungen hat und eventuell dort auch auf die entsprechende Schmerzempfindung (Sullivan 2001), was aber den akuten postoperativen Verlauf angeht, kann das in dieser Studie nicht bestätigt werden.

Auch beim Durchhalteappell, welchen Hasenbring et al. 2001 als Gefahr für Schmerzchronifizierung identifizierten, konnte in dieser Analyse kein Einfluss auf postoperative Schmerzen oder das eingenommene Morphinäquivalent erkannt werden. Man kann also direkt nach der HTEP Operation noch nicht sehen, ob eine mögliche Gefahr der Chronifizierung durch ein starkes Durchhaltevermögen vorhanden ist.

Die Parameter Hilfe- und Hoffnungslosigkeit, der schon von Sullivan 1998 beschrieben wurde, korrelieren sowohl mit den Schmerzangaben postoperativ signifikant, als auch mit dem Analgetikakonsum am 5. postoperativen Tag. Zudem korreliert diese Variable mit dem CPG. Des Weiteren korreliert der KSI Hilfe und Hoffnungslosigkeit noch mit vielen anderen Parametern, wie den drei DASS- und den drei WOMAC-Scores sowie mit dem painDETECT und der durchschnittlichen Schmerzempfindung in den letzten 3 Monaten vor der Operation. 
Aus diesen Verbindungen könnte man einen Circulus vitiosus ableiten (Abbildung 46). Schmerzen hindern Patienten daran, sich in ihrem Alltag gut zu bewegen. Dies führt zu einem Gefühl der Hilfe und Hoffnungslosigkeit, eine Empfindung, welche stark mit der schmerzbezogenen Angst korreliert. Fokussiert der Patient nun seine Gedanken auf mögliche Schmerzen, so bewegt er sich wieder weniger oder nimmt eine mit der Zeit ebenfalls schmerzende Fehlhaltung ein. Das Alter weist bei unserem Patientenkollektiv keine relevante Korrelation zum KSI auf.

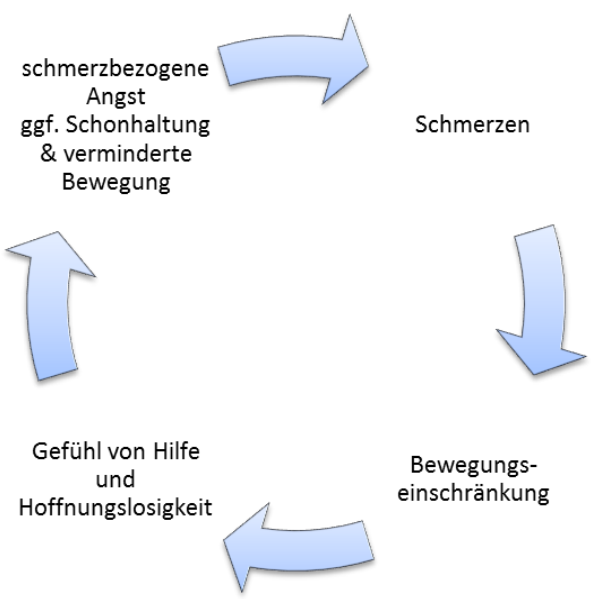

\section{Abbildung 46: Schmerzen - Circulus vitiosus}

Dieser Score der Hilfe und Hoffnungslosigkeit hat dementsprechend bereits im stationären postoperativen Verlauf einen relevanten Einfluss auf das Schmerzempfinden und den Analgetikakonsum, dennoch ist er in diesem Kollektiv nicht multivariat signifikant. Die hohe Interkorrealtion der verschiedenen Konzepte legt auch nahe, dass für Patienten im operativen Setting die hier gewählten Instrumente eine zu geringe Trennschärfe besitzen bzw. einem übergreifenden, unspezifischen und vor allem stressbezogenen Zusammenhang unterliegen könnten, zumal die Befragung am Tag vor der Operation stattfand und womöglich eingeschränkt repräsentativ für die präoperative Lebensperiode ist. 


\subsubsection{Lebensqualität}

In dieser Studie konnten wir nicht bestätigen, dass die körperliche oder psychische Lebensqualität gemessen mit dem SF12 einen signifikanten Einfluss auf postoperative Schmerzen oder eingenommenes Morphinäquivalent nach einer HTEP hat. Dies bestätigt die Ergebnisse von Kalkman et al. 2003, nach denen die Lebensqualität auch keinen signifikanten Einfluss auf den postoperativen Schmerz hatte (Kalkman et al. 2003).

Die körperliche Summenskala bei unserem Patientenkollektiv hat einen um 2 Standardabweichungen schlechteren Wert im Vergleich zu Normalbevölkerung. Demnach kann man durchaus sagen, dass es ein Parameter ist, welcher die Einschränkung des Patientenkollektivs durchaus misst, jedoch ein zu weites Spektrum erfasst, um innerhalb des Kollektives Unterschiede zu detektieren.

Da die psychische Summenskala unseres Patientenkollektivs im Vergleich zur Normalbevölkerung unauffällig blieb, ist dies hinweisend auf den geringen Anteil chronischer Schmerzpatienten in dieser Studie im Vergleich zu anderen Schmerzstudien und im Einklang mit den weitestgehend wenig auffälligen DASS-Werten.

\subsection{3 multivariate Analyse}

Zusammenfassend muss man sagen, dass alle psychologischen Parameter einer hohen Variabilität unterliegen. Sie sind stark tagesformabhängig und weisen insgesamt eine geringe Varianz auf. Man kann zwar einige Tendenzen damit erkennen, aber viele Faktoren sind hierbei nicht identifiziert worden.

\subsection{Angewandte Methodik und Analgesie im Annastift Hannover}

Das Anästhesieverfahren hat einen Einfluss auf postoperative Schmerzen und somit vermutlich auch, langfristig gesehen, auf die Schmerzchronifizerung (Schnabel und Pogatzki-Zahn 2010). So wird von verschiedenen Studien eine regionales Anästhesieverfahren empfohlen, da es kurzfristig, aber auch langfristig gesehen zu einem besseren Outcome führt (Andreae und Andreae 2013, Young und Buvanendran 2014). PROSPECT (Procedure specific postoperative pain management), eine 
Internetplattform, wo nach einer systematischen Literaturrecherche von 1966-2004 Empfehlungen unter anderem für das Anästhesieverfahren bei Hüftendoprothesen angegeben werden, zieht die TIVA ggf. mit lokalen oder spinalen zusätzlichen analgetischen Verfahren der reinen spinalen Anästhesie vor (Fischer und Simanski 2005). Dies ist für eine Folgeuntersuchung interessant, da in dieser Doktorarbeit lediglich die Vollnarkosen in den Datensatz miteinbezogen wurden.

Da akute postoperative Schmerzen die Entwicklung von chronischen Schmerzen stark beeinflussen (Katz et al. 2005; Perkins und Kehlet 2000), sollte eine intensivere Akutschmerztherapie postoperativ erfolgen, um die Entstehung von Schmerzchronifizierungen zu vermindern. So wird von Oreskovic et al. 2014 in den ersten $24 \mathrm{~h}$ postoperativ Metamizol mit einem Opioid empfohlen, da man so die beste Schmerzlinderung erreichen kann. Die Literaturrecherche von PROSPECT ergab zunächst eine Empfehlung für ein NSAR in Kombination mit einem schwachen Opiod (Fischer und Simanski 2005). Im Annastift Hannover wurde eine ähnliche Kombination mit Ibuprofen und niedrigdosiertes Oxycodon angewandt, wodurch ebenfalls eine gute Schmerzlinderung erzielt werden konnte.

Eine Kontrollmöglichkeit postoperativ für die angewandte Analgetikatherapie sollte konsequenter durchgeführt werden. Mit der Einführung von QUIPS hat sich bereits in einigen Krankenhäusern die Qualität des Akutschmerzdienstes verbessert (Meissner et al. 2008). Der Aufwand ist im normalen Krankenhausalltag einfach integrierbar und dient sowohl als Rückmeldung an den verordnenden Arzt als auch der Fehlererkennung im System. Es gibt also dem Akutschmerzdienst die Möglichkeit einer Qualitätsverbesserung durch ein standardisiertes Feedback. Natürlich ist ein solcher Fragebogen nur sinnvoll, wenn man sich den detektierten Lücken aufmerksam widmet und der Akutschmerzdienst postoperativ entsprechend ausgeweitet wird (Erlenwein et al. 2013; Schnabel und Pogatzki-Zahn 2010). Wie zu Beginn der Doktorarbeit beschrieben, besteht bereits ein standardisiertes Analgesie-Schema, mit dem die Patienten im Haus behandelt werden. Der QUIPS-Fragebogen wäre ein ergänzendes Mittel, welches in der Visite mögliche unerwünschte Nebenwirkungen der angewandten Medikation standardisiert detektiert. 
Präoperative körperliche Übungen reduzieren postoperative Akutschmerzen und verbessern das postchirurgische Outcome. Da Patienten häufig auf eine elektive HTEP Operation warten, kann man gerade diese Wartezeit nutzen, um die Patienten auf ihre neue Hüfte vorzubereiten (Wallis und Taylor 2011). Der Zeitpunkt, wann eine Operation durchgeführt wird, sollte der funktionellen Aktivität besser angepasst werden, da eine zu späte Durchführung den Prozess die körperliche Wiederherstellung verzögert (Lavernia et al. 2009). Im Annastift Hannover wurde präoperativ eine Gewichtsabnahme bei adipösen Patienten empfohlen. Da dies nicht weiter im Rahmen der Studie untersucht wurde, wäre hier ein möglicher Ansatz für Folgeuntersuchungen. Wir untersuchten hingegen die unmittelbare präoperative Mobilität mittels des Timed „Up and Go“-Tests sowie des "Treppengehversuches“. Dort stellten wir zwar univariat eine Signifikanz beider Parameter auf den akuten Schmerz am 5. Postoperativen Tag fest, jedoch sind Parameter in der multiplen Regression nicht mehr signifikant, sodass wir eine Korrelation bestätigen können, jedoch die Stärke des Einflusses der Parameter gering ausfällt.

Insgesamt gesehen kann man sagen, dass eine intensive präoperative Aufklärung besonders wichtig ist. Damit kann man nicht nur die Angst vor einer Opioidabhängigkeit reduzieren und vielleicht einen geeigneten Zeitpunkt für die Operation festlegen, sondern auch durch eine verkürzte stationäre Aufenthaltsdauer die Kosten für das Gesundheitssystem senken (Birrell et al. 1999).

\subsection{Einschränkungen der Studie}

Obwohl die Operationen ausschließlich im Annastift Hannover durchgeführt wurden, lassen sich Unterschiede im operativen Vorgehen nicht gänzlich vermeiden, so dass man von dieser Seite einen individuellen Einfluss auf das postoperative Ergebnis nicht ausschließen kann.

Das Patientenkollektiv ist mit 125 Patienten relativ klein. 
Es wurde ausschließlich die primäre elektive HTEP betrachtet, was zwar eine homogene Betrachtung ermöglicht, aber die Vielfalt an sekundären HTEP-Operationen und Frakturen außer Acht lässt.

Leider war die Vergleichsgruppe der spinalen durchgeführten Anästhesie mit 7 Patienten zu klein, um sie mit diesem Patientenkollektiv adäquat vergleichen zu können.

Interessant ist die Nebenbeobachtung, dass die Bettnachbarn einen großen Einfluss auf die psychische Verfassung während des Krankenhausaufenthaltes der Patienten hatten. Auch der Umgang auf der Station von den Angestellten schlug sich wahrnehmbar auf die Stimmung der Patienten nieder. $\mathrm{Da}$ in dieser Studie aber nur die genannten Prädiktoren untersucht wurden, fließen diese Faktoren höchstens sekundär in die Schmerzempfindung am 5. postoperativen Tag mit ein. Den Autoren sind keine Studien bezüglich des Einflusses von Bettnachbarn und wie man das messen/erfassen kann bekannt. 


\section{Zusammenfassung}

Die Identifikation von chronischen Schmerzpatienten und in diesem Fall chronischen postoperativen Schmerzpatienten sollte möglichst früh diagnostiziert werden, um eine adäquate Behandlung durchführen zu können (Kehlet et al. 2006). Schmerzen machen Patienten unzufrieden, was ein schlechteres Outcome zur Folge haben kann (Anakwe et al. 2011). Da dieses scheinbar stark vom prä-, peri- und postoperativen Verlauf sowie der kognitiven und psychischen Veranlagung der Patienten abhängig ist, sollten diese Faktoren mehr Beachtung finden. In dieser Doktorarbeit wurde das Augenmerk auf den akuten postoperativen Verlauf gelegt.

Es wurden diverse präoperative Faktoren identifiziert, welche einen Einfluss auf akute postoperative Schmerzen und den akuten Morphingebrauch postoperativ beeinflussen. Aufgrund der multifaktoriellen Einflüsse, ist es notwendig, eine geeignete Vorauswahl an möglichen Testungen zu treffen. Da depressiv veranlagte Personen sowohl eine Veranlagung für stärkere postoperative Schmerzen, als auch einen erhöhten Morphingebrauch aufweisen, ist hier eine sinnige Testung präoperativ gegeben, obwohl die Anzahl der auffälligen Patienten in diesem untersuchten Kollektiv sehr gering war. Auch der individuell wahrgenommene Stress sollte zuvor erhoben werden, um ggf. stresslindernde Maßnahmen ergreifen zu können, da auch dieser Parameter signifikanten Einfluss auf die postoperativen Schmerzen hatte.

Zur Detektion der präoperativen Funktion bei HTEP-Patienten eignet sich der Timed „Up and Go“-Test sowie der WOMAC-Fragebogen.

Der painDETECT, eigentlich ein Parameter zur Detektion neuropathischer Schmerzen, weist in dieser Arbeit eine so hohe Signifikanz bei den postoperativen Schmerzen auf, dass man diskutieren muss, inwiefern dieser Fragebogen auch bei orthopädischen Eingriffen eingesetzt werden sollte. Mutmaßlich ist also eine Veranlagung für erhöhten postoperativen Schmerz bei Patienten gegeben, welche eine erhöhte Empfindlichkeit auch gegenüber neuropathischen Schmerzen aufweisen. Möglich weitere Diagnosen sind bei unserem Patientenkollektiv nicht auszuschließen, da Nebenerkrankungen, wie Fibromyalgie, im Rahmen dieser Studie nicht erhoben wurden. 
Zuletzt sei noch erwähnt, dass sich sowohl der MPSS als auch der CPG teilweise als signifikant herausstellten. In weiteren Studien wäre es vermutlich sinnvoll, den CPG bei der Durchführung eines Hüftgelenksersatzes zu verwenden, da er zum einen flexibler in den Chronifizierungsstadien ist und zum anderen auch die Funktionalität des Patienten miteinbezieht. 


\section{Literaturverzeichnis}

Aasvang $\mathrm{E}$, Kehlet $\mathrm{H}$ (2005): Chronic postoperative pain: the case of inguinal herniorrhaphy. $\mathrm{Br} \mathrm{J}$ Anaesth $\underline{95}$, 69-76

Abrishami A, Chan J, Chung F, Wong J (2011): Preoperative Pain Sensitivity and Its Correlation with Postoperative Pain and Analgesic Consumption: A Qualitative Systematic Review. Anaesthesiology 114, 445457

Althaus A, Hinrichs-Rocker A, Chapman R, Arránz Becker O, Lefering R, Simanski C, Weber F, Moser K-H, Joppich R, Trojan S, Gutzeit N, Neugebauer E (2012): Development of a risk index for the prediction of chronic post-surgical pain. Eur J Pain $\underline{16}, 901-910$

American Geriatrics Society Panel on the Pharmacological Management of Persistent Pain in Older Persons (2009): Pharmacological Management of Persistent Pain in Older Persons. J Am Geriatr Soc $\underline{57}$, 1331-46

Anakwe RE, Jenkins PJ, Moran M (2011): Predicting Dissatisfaction After Total Hip Arthroplasty: A Study of 850 Patients. J Arthroplasty $\underline{26}, 209-213$

Andreae MH, Andreae DA (2013): Regional anaesthesia to prevent chronic pain after surgery: a Cochrane systematic review and meta-analysis. $\mathrm{Br} \mathrm{J}$ Anaesth $\underline{111}, 711-20$

Atkinson HD, Johal KS, Willis-Owen C, Zadow S, Oakeshott RD (2010): Differences in hip morphology between the sexes in patients undergoing hip resurfacing. J Orthop Surg Res $\underline{5}, 76$

Batsis JA, Naessens JM, Keegan MT, Wagie AE, Huddleston PM, Huddleston JM (2009): Impact of body mass on hospital resource use in total hip Arthroplasty. Public Health Nutr $\underline{12}, 1122-1132$

Bellamy N, Buchanan WW, Goldsmith CH, Campbell J, Stitt LW (1988): Validation study of WOMAC: a health status instrument for measuring clinically important patient relevant outcomes to antirheumatic drug therapy in patients with osteoarthritis of the hip or knee. J Rheumatol 12, 1833-1840.

Bener A, Verjee M, Dafeeah EE, Falah O, Al-Juhaishi T, Schlogl J, Sedeeq A, Khan S (2013): Psychological factors: anxiety, depression, and somatization symptoms in low back pain patients. J Pain Res $\underline{6}, 95-101$

Birrell F, Johnell O, Silman A (1999): Projecting the need for hip replacement over the next three decades: influence of changing demography and threshold for surgery. Ann Rheum Dis $\underline{58}, 569-572$

Bijlsma JWJ, Berenbaum F, Lafeber FPJG (2011): Osteoarthritis: an update with relevance for clinical practice. Lancet $\underline{377}, 2115-2126$

Brander VA, Stulberg SD, Adams AD, Harden RN, Bruehl S, Stanos SP, Houle T (2003): Predicting total knee replacement pain: a prospective, observational study. Clin Orthop Relat Res $\underline{16}$, 27-36

Breivik H, Collett B, Ventafridda V, Cohen R, Gallacher D (2006): Survey of chronic pain in Europe: Prevalence, impact on daily life, and treatment. Eur J Pain 10, 287-333

Bullinger M, Kirchberger I: Handanweisung SF-12, Kurzversion des SF-36. In: Bullinger M, Kirchberger I (Hrsg.): SF-36, Fragebogen zum Gesundheitszustand. Hogrefe Verlag, Göttingen 1998, S.68

Busato A, Röder C, Herren S, Eggli S (2008): Influence of High BMI on Functional Outcome after Total Hip Arthroplasty. Obes Surg 18, 595-600

Chouchou F, Khoury S, Jean-Marc Chauny J-M, Denis R, Lavigne G J (2014): Postoperative sleep disruptions: A potential catalyst of acute pain? Sleep Med Rev $\underline{18}, 273-282$

Chung F, Ritchie E, Su J (1997): Postoperative Pain in Ambulatory Surgery. Anesth Analg $\underline{85}$, 808-816 
Ene KW, Nordberg G, Johansson F G, Sjöström B (2006): Pain, psychological distress and health-related quality of life at baseline and 3 months after radical prostatectomy. BMC Nurs $\underline{5}, 8$

Erlenwein J, Schlink J, Pfingsten M, Hinz J, Bauer M, Quintel M, Petzke F (2013): Vorbestehender Schmerz als Komorbidität im postoperativen Akutschmerzdienst. Anaesthesist $\underline{62}, 808-816$

Erlenwein J, Przemeck M, Degenhart, A, Budde S, Falla D, Quintel M, Pfingsten M, Petzke F (2016): The Influence of Chronic Pain on Postoperative Pain and Function After Hip Surgery: A Prospective Observational Cohort Study. J Pain 17, 236-247

Etherton J, Lawson M, Graham R (2014): Individual and Gender Differences in Subjective and Objective Indices of Pain: Gender, Fear of Pain, Pain Catastrophizing and Cardiovascular Reactivity. Appl Psychophysiol Biofeedback $\underline{39}, 89-97$

Fillingim RB, King CD, Ribeiro-Dasilva MC, Rahim-Williams B, Riley JL (2009): Sex, Gender, and Pain: A Review of Recent Clinical and Experimental Findings. J Pain 10, 447-458

Fischer HBJ, Simanski CJP (2015): A procedure-specific systematic review and consensusrecommendations for analgesia after total hip replacement. Anaesthesia $\underline{60}, 1189-1202$

Fortin PR, Clarke AE, Joseph L, Liang MH, Tanzer M, Ferland D, Phillips C, Partridge AJ, Bélisle P, Fossel AH, Mahomed N, Sledge CB, Katz JN (1999): Outcomes of total hip and knee replacement: preoperative functional status predicts outcomes at six months after surgery. Arthritis Rheum $\underline{42}, 1728$

Frettlöh J, Maier C, Gockel H, Hüppe M (2003): Validität des Mainzer Stadienmodells der Schmerzchronifizierung bei unterschiedlichen Schmerzdiagnosen. Schmerz 17, 240-251

Frettlöh J, Maier C, Gockel H, Zenz M, Hüppe M (2009): Patientenkollektiv deutscher schmerztherapeutischer Einrichtungen - Kerndaten von mehr als 10.000 Patienten. Schmerz 23, 576 - 591

Freynhagen R, Baron R, Gockel U, Tölle TR (2006): painDETECT: a new screening questionnaire to identify neuropathic components in patients with back pain. Curr Med Res Opin 22, 1911-1920

Gandek B, Ware JE, Aaronson NK, Apolone G, Bjorner JB, Brazier JE, Bullinger M, Kaasa S, Leplege A, Prieto L, Sullivan M (1998): Cross-Validation of Item Selection and Scoring for the SF-12 Health Survey in Nine Countries: Results from the IQOLA Project. J Clin Epidemiol $\underline{51}$, 1171-1178

Geiss A, Rohleder N, Kirschbaum C, Steinbach K, Bauer HW, Anton F (2005): Predicting the failure of disc surgery by a hypofunctional HPA axis: evidence from a prospective study on patients undergoing disc surgery. Pain $\underline{114}, 104-117$

Gerbershagen HU (1986): Organisierte Schmerzbehandlung. Eine Standortbestimmung. Internist $\underline{27}, 459-469$

Gerbershagen HU, Aduckathil S, van Wijck A J, Peelen L M, Kalkman C J, Meissner W (2013): Pain intensity on the first day after surgery: a prospective cohort study comparing 179 surgical procedures. Anaesthesiology $\underline{118}$, 934-944

Gouin J-P, Kiecolt-Glaser JK (2011): The Impact of Psychological Stress on Wound Healing: Methods and Mechanisms. Immunol Allergy Clin North Am $\underline{31}, 81-93$

Häuser W, Wolfe F, Hennigsen P, Schmutzer G, Brähler E, Hinz A (2014) Untying chronic pain: prevalence and societal burden of chronic pain stages in the general population - a cross-sectional survey. BMC Public Health $\underline{14}, 352$

Hampel P, Moergel MF (2009): Schmerzchronifizierung bei Rückenschmerzpatienten in der stationären Rehabilitation - Zur Validität des Mainzer Stadienmodells der Schmerzchronifizierung. Schmerz 4, 154-165 
Hanlon JT, Backonja M, Weiner D, Argoff C (2009): Evolving Pharmacological Management of Persistent Pain in Older Persons. Pain Med 10, 959-961

Harden RN, Weinland SR, Remble TA, Houle TT, Colio S, Steedman S, Kee WG, American Pain Society Physicians (2005): Medication Quantification Scale Version III. Update in medication classes and revised detriment weights by survey of American Pain Society Physicians. J Pain $\underline{6}, 364-371$

Hasenbring M (1993): Durchhaltestrategien - ein in Schmerzforschung und Therapie vernachlässigtes Phänomen? Schmerz 12, 304-313

Hasenbring M, Hallner D, Klasen B (2001): Psychologische Mechanismen im Prozess der Schmerzchronifizierung. Schmerz $\underline{15}, 442-447$

Huang N, Cunningham F, Laurito CE, Chen C (2001): Can we do better with postoperative pain management? Am J Surg 182, 440-448

Hüppe M, Maier C, Gockel H, Zenz M, Frettlöh J (2011): Behandlungserfolg auch bei höherer Schmerzchronifizierung? - Eine Auswertung des Mainzer Stadienmodells auf Basis der QUASTAnalysestichprobe. Schmerz $\underline{25}, 77-88$

Lavand'homme P, Pogatzki-Zahn E: Chronic Postsurgical Pain: Definition, Impact, and Prevention. IASP 2017 http://s3.amazonaws.com/rdcms-

iasp/files/production/public/2017GlobalYear/FactSheets/4.\%20Chronic\%20Postsurgical\%20Pain.LavandHomm e-Zahn-EE_1485789834697_3.pdf, Zugriff am 13.05.2018

Ibrahim MS, Twaij H, Giebaly DE, Nizam I, Haddad FS (2013): Enhanced recovery in total hip replacement. Bone Joint J $\underline{95-B}, 1587-1594$

Innocenti M, Nistri L, Biondi M, del Prete A, Giorgini M, Macera A, Soderi S (2013): Hip arthrosis and surgical intervention: what and when? Clin Cases in Miner Bone Metab 10, 41-46

Ip HYV, Abrisham A, Peng PWH, Wong J, Chung F (2009): Predictors of Postoperative Pain and Analgesic Consumption - A Qualitative Systematic Review. Anesthesiology 111, 657-677

Jüni P, Low N, Reichenbach S, Villiger PM, Williams S, Dieppe PA (2010): Gender inequity in the provision of care for hip disease: population-based Cross-sectional study. Osteoarthritis Cartilage $\underline{18}, 640-645$

Kalkman CJ, Visser K, Moen J, Bonsel GJ, Grobbee DE, Moons KGM (2003): Preoperative prediction of severe postoperative pain. Pain $\underline{105}, 415-423$

Katz J, Poleshuck EL, Andrus CH, Hogan LA, Jung BF, Kulick DI, Dworkin RH (2005): Risk factors for acute pain and its persistence following breast cancer surgery. Pain $\underline{119}, 16-25$

Kain ZN, Sevarino F, Alexander GM, Pincus S, Mayes LC (2000): Preoperative anxiety and postoperative pain in women undergoing hysterectomy - A repeated-measures design. J Psychosom Res $\underline{49}$, 417-422

Kehlet H, Rung GW, Callesen T (1996): Postoperative Opioid Analgesia: Time for a Reconsideration? J Clin Anesth $\underline{8}, 441-445$

Kehlet H, Jensen TS, Woolf CJ (2006): Persistent postsurgical pain: risk factors and prevention. Lancet $\underline{367}$, $1618-1625$

Kessler S, Käfer W (2007): Overweight and Obesity: Two Predictors for Worse Early Outcome in Total Hip Replacement? Obesity 15 , 2840-2845

Khan RS, Ahmed K, Blakeway E, Skapinakis P, Nihoyannopoulos L, Macleod K, Sevdalis N, Ashrafian H, Platt M, Darzi A, Athanasiou T (2011): Catastrophizing: a predictive factor for postoperative pain. Am J Surg 201, $122-131$ 
Klasen BW, Hallner D, Schaub C, Willburger R, Hasenbring M (2004): Validation and reliability of the German version of the Chronic Pain Grade questionnaire in primary care back pain patients. Psychosocial Med 1 , Doc07

Koroschetz J, Rehm SE, Gockel U, Brosz M, Freynhagen R, Tölle TR, Baron R (2011): Fibromyalgia and neuropathic pain - differences and similarities. A comparison of 3057 patients with diabetic painful neuropathy and fibromyalgia. BMC Neurol $\underline{11}, 55$

Kroenke K, Spitzer RL, Williams JB (2002): The PHQ-15: Validity of a new measure for evaluating the severity of somatic symptoms. Psychosom Med $\underline{64}, 258-266$

Lautenbacher S, Huber C, Kunz M, Parthum A, Weber P, Griessinger N, Sittl R (2009): Hypervigilance as Predictor of Postoperative Acute Pain: Its Predictive Potency Compared With Experimental Pain Sensitivity, Cortisol Reactivity, and Affective State Clin J Pain 25, 92-100

Lavernia CJ, D'Apuzzo M, Rossi MD, Lee D (2009): Is Postoperative Function After Hip or Knee Arthroplasty Influenced by Preoperative Functional Levels? J Arthroplasty 24, 1033-1043

Lavernia CJ, Alcerro JC, Contreras JS, Rossi MD (2011): Patient Perceived Outcomes After Primary Hip Arthroplasty - Does Gender matter? Clin Orthop Relat Res $\underline{469}$, 348-354

Lecerf G, Fessy MH, Philippot R, Massin P, Giraud F, Fletcher X, Giraud J, Mertl P, Marchetti E, Stindel E (2009): Femoral offset: Anatomical concept, definition, assessment, implications for preoperative templating and hip arthroplasty. Orthop Traumatol Surg Res 95, 210-219

LeResche L (2011): Defining Gender Disparities in Pain Management. Clin Orthop Relat Res $\underline{469}, 1871-1877$

Lethem J, Slade PD, Troupe JDG, Bentley G (1983): Outline of a Fear-Avoidance Model of exaggerated pain perception - I. Behav Res Ther $\underline{21}$, 401-408.

Liu SS, Buvanendran A, Rathmell JP, Sawhney M, Bae JJ, Moric M, Perros S, Pope AJ, Poultsides L, Della Valle CJ, et al. (2012): Predictors for moderate to severe acute postoperative pain after total hip and knee replacement. Int Orthop $\underline{36}, 2261-2267$

Lovibond PF, Lovibond SH (1995): The structure of negative emotional states: comparison of the Depression Anxiety Stress Scales (DASS) with the Beck Depression and Anxiety Inventories. Behav Res Ther $\underline{3}$, 335-343

Luman W, Adams WH, Nixon SN, Mcintyre IM, Hamer-Hodges D, Wilson G, Palmer KR (1996): Incidence of persistent symptoms after laparoscopic cholecystectomy: a prospective study. Gut $\underline{39}, 863-866$

Macrae WA (2001): Chronic Pain After Surgery. Br J Anaesth 무, 88-98

Mallick-Searle T, Snodgrass B, Brant JM (2016): Postherpetic neuralgia: epidemiology, pathophysiology, and pain management pharmacology. J Multidiscip Healthc $\underline{9}$, 447-454

Mathias S, Nayak U, Isaacs M (1986): Balance in elderly patients: the 'get-up and go-test'. Arch Phys Med Rehabil $\underline{67}, 387-389$

Meissner W, Ullrich K, Zwacka S (2006): Benchmarking as a tool of continuous quality improvement in postoperative pain management, Eur J Anaesthesiol $\underline{23}$, 142-148

Meissner W, Mescha S, Rothaug J, Zwacka S, Goettermann A, Ulrich K, Schleppers A (2008): Quality Improvement in Postoperative Pain Management. Dtsch Arztebl Int 105, 865-870

Mensink GBM, Schienkiewitz A, Haftenberger M, Lampert T, Ziese T, Scheidt-Nave C (2013): Übergewicht und Adipositas in Deutschland - Ergebnisse der Studie zur Gesundheit Erwachsener in Deutschland (DEGS1).

Bundesgesundheitsblatt Gesundheitsforschung Gesundheitsschutz $\underline{56}, 786-794$ 
Merle C, Waldstein W, Gregory JS, Goodyear SR, Aspen RM, Aldinger PR, Murray DW, Gill HS (2014): How Many Different Types of Femora are There in Primary Hip Osteoarthritis? An Active Shape Modeling Study. J Orthop Res $\underline{32}, 413-422$

Merx H, Dreinhöfer K, Schräder P, Stürmer T, Puhl W, Günther K-P, Brenner H (2003): International variation in hip replacement rates. Ann Rheum Dis $\underline{62}, 222-226$

Miller R, Kori S, Todd DD (1991): The Tampa Scale a measure of Kinesiophobia. Clin J Pain $\underline{7}, 51-52$

Montin L, Leino-Kilpi H, Suominen T, Lepisto J (2008): A systematic review of empirical studies between 1966 and 2005 of patient outcomes of total hip arthroplasty and related factors. J Clin Nurs $\underline{17}, 40-45$

Munafo MR, Stevenson J (2001): Anxiety and surgical recovery - Reinterpreting the literature. J Psychosom Res $\underline{51}, 589-596$

Myles PS, Williams DL, Hendrata M, Anderson H, Weeks AM (2000): Patient satisfaction after anaesthesia and surgery: results of a prospective survey of 10811 patients. $\mathrm{Br} \mathrm{J}$ Anaesth $\underline{84}, 6-10$

Nagel B, Pfingsten M, Lindena G, Kohlmann T (2015): Handbuch zum Deutschen Schmerzfragebogen. 3 Version; DGSS. https://www.dgss.org/fileadmin/user_upload/DSF-Handbuch_2015.pdf, Zugriff am 29.09.2018

Nikolajsen L, Brandsborg B, Lucht U, Jensen TS, Kehlet H (2006): Chronic pain following total hip arthroplasty: a nationwide questionnaire study. Acta Anaesthesiol Scand $\underline{50}, 495-500$

Nilges P, Essau C (2015): Die Depressions-Angst-Stress-Skalen - Der DASS - ein Screeningverfahren nicht nur für Schmerzpatienten. Schmerz $\underline{29}, 649-657$

Nilsdotter A-K, Petersson IF, Roos EM, Lohmander LS (2003): Predictors of patient relevant outcome after total hip replacement for osteoarthritis: a prospective study. Ann Rheum Dis $\underline{62}, 923-930$

Oreskovic Z, Bicanic G, Hrabac P, Tripkovic B, Delimar D (2014): Treatment of postoperative pain after total hip arthroplasty: comparison between metamizol and paracetamol as adjunctive to opioid analgesics - prospective, double-blind, randomized study. Arch Orthop Trauma Surg 134, 631-636

Pacault-Legendre V, Anract P, Mathieu M, Courpied JP (2009): Pain after total hip arthroplasty: a psychiatric point of view. Int Orthop $\underline{33}, 65-69$

Paul JE, Manyat N-A, Buckley N, Shahzad U, Cheng J, Thabane L, Tidy A, DeBeer J, Winemaker M, Wismer D et al. (2015): Randomized controlled trial of gabapentin as an adjunct to perioperative analgesia in total hip arthroplasty patients. J Can Anesth $\underline{62,476-484}$

Perkins FM, Kehlet H (2000): Chronic Pain as an Outcome of Surgery - A Review of Predictive Factors. Anesthesiology $\underline{93}, 1123-1133$

Peters ML, Sommer M, de Rijke JM, Kessels F, Heineman E, Patijn J, Marcus MAE, Vlaeyen JWS, van Kleef M (2007): Somatic and Psychologic Predictors of Long-term Unfavorable Outcome After Surgical Intervention. Ann Surg 245, 487-494

Petersen KK, Arendt-Nielsen L, Simonsen O, Wilder-Smith O, Berg Laursen M (2015): Presurgical assessment of temporal summation of pain predicts the development of chronic postoperative pain 12 months after total knee replacement. Pain $156,55-61$

Petre BM, Roxbury CR, McCallum JR, DeFontes KW, Belkoff SM, Mears SC (2012): Pain Reporting, Opiate Dosing, and the Adverse Effects of Opiates After Hip or Knee Replacement in Patients 60 Years Old or Older. Geriatr Orthop Surg Rehabil $\underline{3}, 3-7$

Pivec R, Johnson AJ, Mears SC, Mont MA (2012): Hip arthroplasty. Lancet $\underline{380}$, 1768-1777 
Pivec R, Issa K, Naziri Q, Kapadia BH, Bonutti PM, Mont MA (2014): Opioid use prior to total hip arthroplasty leads to worse clinical outcomes. Int Orthop $\underline{38}, 1159-1165$

Podsiadlo D, Richardson S (1991): The timed "Up \& Go": a test of basic functional mobility for frail elderly persons. J Am Geriatr Soc 39, 142-148

Riediger W, Doering S, Krismer M (2010): Depression and somatisation influence the outcome of total hip replacement. Int Orthop $\underline{34}, 13-18$

Ruau D, Liu LY, Clark JD, Angst MS, Butte AJ (2012): Sex Differences in Reported Pain Across 11,000 Patients Captured in Electronic Medical Records. J Pain 13, 228-234

Rusu AC, Kreddig N, Hallner D, Hülsebusch J, Hasenbring M (2014): Fear of movement/(Re)injury in low back pain: confirmatory validation of a German version of the Tampa Scale for Kinesiophobia. BMC Musculoskelet Disord $\underline{15}, 280$

Schäfer AGM, Joos L J, Roggemann K, Waldvogel-Röcker K, Pfingsten M, Petzke F (2017): Pain experiences of patients with musculoskeletal pain + central sensitization: A comparative Group Delphi Study. PLoS One 12 e0182207

Schnabel A, Pogatzki-Zahn E (2010): Prädiktoren für chronische Schmerzen nach Operationen. Schmerz $\underline{24}$, 517-533

Schneider M, Kawahara I, Ballantyne G, McAuley C, MacGregor K, Garvie R, McKenzie A, MacDonald D, Breusch SJ (2009): Predictive factors influencing fast track rehabilitation following primary total hip and knee arthroplasty. Arch Orthop Trauma Surg $\underline{129}$, 1585-1591

Schulz A, Locher H (2013): Schmerz am Bewegungsorgan verstehen, untersuchen und diagnostizieren. Orthopäde $\underline{42}, 854-857$

Shaygan M, Böger A, Kröner-Herwig B (2017): Clinical features of chronic pain with neuropathic characteristics: a symptom-based assessment using the pain DETECT questionnaire. Eur J Pain 10,1529-1538

Singh JA, Lewallen D (2010): Predictors of pain and use of pain medications following primary Total Hip Arthroplasty (THA): 5,707 THAs at 2-years and 3,289 THAs at 5-years. BMC Musculoskelet Disord 11, 90

Smith BH, Penny KI, Purves AM, Munro C, Wilson B, Grimshaw J, Chambers WA, Smith WC (1997): The Chronic Pain Grade questionnaire: validation and reliability in postal research. Pain $\underline{71}, 141-147$

Solomon DH, Rassen JA, Glynn RJ, Garneau K, Levin R, Lee J, Schneeweiss S (2010): The Comparative Safety of Opioids for Nonmalignant Pain in Older Adults. Arch Intern Med $\underline{170}$, 1979-1986

Spitzer RI, Kroenke K, Williams JB (1999): Validation and Utility of a Self-report Version of PRIME-MD - The PHQ Primary Care Study. JAMA 282, 1737- 1744

Sullivan MJL, Stanish W, Waite H, Sullivan M, Tripp DA (1998): Catastrophizing, pain, and disability in patients with soft-tissue injuries. Pain $\underline{77}, 253-260$

Sullivan MJ, Rodgers WM, Kirsch I (2001): Catastrophizing, depression and expectancies for pain and emotional distress. Pain $\underline{91}, 147-154$

Turk DC, Okifuji A (1996): Perception of Traumatic Onset, Compensation Status, and Physical Findings: Impact on Pain Severity, Emotional Distress, and Disability in Chronic Pain Patients. J Behav Med 19, 435-453

van Laarhoven AIM, Kraaimaat FW, Wilder-Smith $\mathrm{OH}$, van de Kerkhof PCM, Cats $\mathrm{H}$, van Riel PLCM, Evers AWM (2007): Generalized and symptom-specific sensitization of chronic itch and pain. Eur Acad Dermatol Venereol $\underline{21}, 1187-1192$ 
Vlaeyen JWS, Linton SJ (2000): Fear-avoidance and its consequences in chronic musculoskeletal pain: a state of the art. Pain $\underline{85}, 317-332$

Vlaeyen JWS, Kole-Snijders AMJ, Boeren RGB, van Eek H (1995): Fear of movement/ (re) injury in chronic low back pain and its relation to behavioral performance. Pain $\underline{62}, 363-372$

Von Korff M, Ormel J, Keefe FJ, Dworkin SF (1992): Grading the severity of chronic pain. Pain $\underline{50}$, 133-149

Wallis JA, Taylor NF (2001): Pre-operative interventions (non-surgical and non-pharmacological) for patients with hip or knee osteoarthritis awaiting joint replacement surgery - a systematic review and meta-analysis. Osteoarthritis Cartilage $\underline{19}, 1381-1395$

Ware JE Jr, Kosinski M, Keller SD (1996): A 12-Item Short-Form Health Survey: Construction of Scales and Preliminary Tests of Reliability and Validity. Med Care $\underline{3}, 220-233$

Wood BM, Nicholas MK, Blyth F, Asghari A, Gibson S (2010): The Utility of the Short Version of the Depression Anxiety Stress Scales (DASS-21) in Elderly Patients with Persistent Pain: Does Age Make a Difference? Pain Med 11, 1780-1790

Wylde V, Rooker J, Halliday L, Blom A (2011a): Acute postoperative pain at rest after hip and knee arthroplasty: Severity, sensory qualities and impact on sleep. Orthop Traumatol Surg Res $\underline{97}, 139-144$

Wylde V, Hewlett S, Learmonth ID, Dieppe P (2011b): Persistent pain after joint replacement: Prevalence, sensory qualities, and postoperative determinants. Pain $\underline{152}, 566-572$

Young AC, Buvanendran A (2014): Pain Management for Total Hip Arthroplasty. J Surg Orthop Adv $\underline{23}, 13-21$

Zeni J Jr, Abujaber S, Pozzi F, Raisis L (2014): Strength and pain are related to different measures of functional ability in patients with end-stage hip osteoarthritis. Arthritis Care Res $\underline{66}, 1506-1512$ 